FEMP-2552

UC-702

THE COMPARISON OF CAP88-PC VERSION 2.0

VERSUS CAP88-PC VERSION 1.0

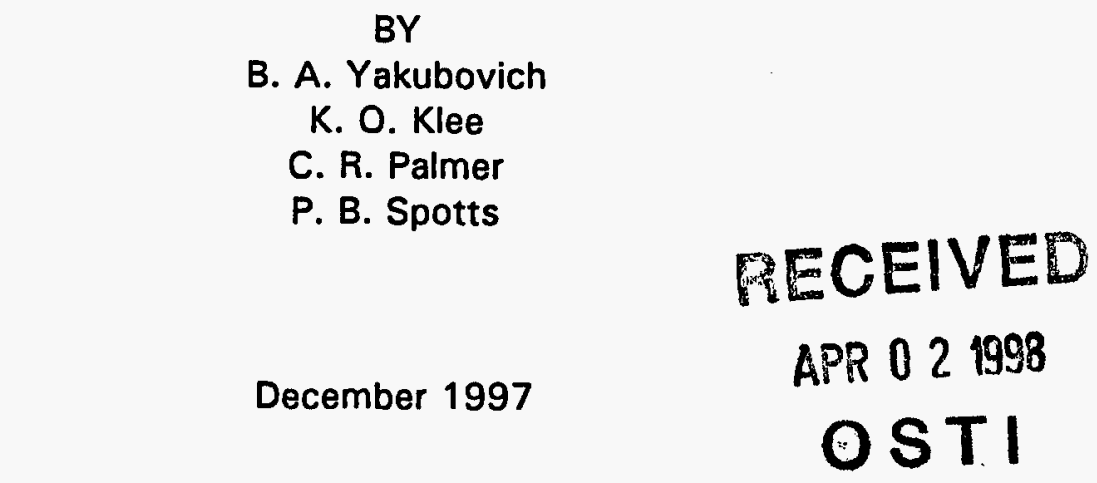

Fluor Daniel Fernald, Inc.

Fernald Environmental Management Project

P.O. Box 538704

Cincinnati, OH 45253-8704

DISTRIBUTION OF THIS DOCUMENT IS UNLIMITEB

DIC QUALTH INESRCIED

Prepared for the

U.S. Department of Energy

Fernald Field Office

Under Contract DE-AC24-920R21972 


\section{DISCLAIMER}

This report was prepared as an account of work sponsored by an agency of the United States Government. Neither the United States Government nor any agency thereof, nor any of their employees, makes any warranty, express or implied, or assumes any legal liability or responsibility for the accuracy, completeness, or usefulness of any information, apparatus, product, or process disclosed, or represents that its use would not infringe privately owned rights. Reference herein to any specific commercial product, process, or service by trade name, trademark, manufacturer, or otherwise does not necessarily constitute or imply its endorsement, recommendation, or favoring by the United States Government or any agency thereof. The views and opinions of authors expressed herein do not necessarily state or reflect those of the United States Government or,any agency thereof. 


\title{
THE COMPARISON OF CAP88-PC VERSION 2.0 VERSUS CAP88-PC VERSION 1.0
}

\author{
BY \\ B. A. Yakubovich, FDF \\ K. O. Klee, FDF \\ C. R. Palmer, FDF \\ P. B. Spotts, FDF \\ December, 1997 \\ Facility: Fernald Environmental Management Project \\ Address: P.O. Box 398704 \\ 7400 Willey Road \\ City: Cincinnati \\ State: OH Zip: $45253-8704$ \\ Division: Facility Closure and Demolition Project \\ Department: Environmental Compliance
}

*FDF with the U.S. Department of Energy, under Contract No. DE-AC24-920R21972 
TABLE OF CONTENTS

SECTION IITLE PAGE

1.0 EXECUTIVE SUMMARY $\ldots \ldots \ldots \ldots \ldots \ldots$

$2.0 \quad$ INTRODUCTION $\ldots \ldots \ldots \ldots \ldots \ldots \ldots$

EVALUATION PROCEDURE $\ldots \ldots \ldots \ldots \ldots$

RESULTS AND DISCUSSION $\ldots \ldots \ldots \ldots$

$5.0 \quad$ CONCLUSIONS. ............... 5

6.0 RECOMMENDATIONS $\ldots \ldots \ldots \ldots \ldots \ldots$

\section{LIST OF TABLES}

IABLE IITLE PAGE

1 CAP88-PC INPUT DATASETS $\ldots \ldots \ldots \ldots \ldots$

2 CAP88-PC MEI OUTPUT SUMMARY ............ 5

LIST OF ATTACHMENTS

ATTACHMENT A - DATASET \#1, CAP88-PC $\vee 1.0$ AND $v 2.0$ SYNOPSIS AND SUMMARY FILES

ATTACHMENT B - DATASET \#2, CAP88-PC 1.0 AND $\vee 2.0$ SYNOPSIS AND SUMMARY FILES

ATTACHMENT C - DATASET \#3, CAP88-PC 1.0 AND $\vee 2.0$ SYNOPSIS AND SUMMARY FILES 


\subsection{EXECUTIVE SUMMARY}

The personal computer software program CAP88-PC was developed at the Department of Energy (DOE) for use in calculating dose and risk values from facilities that emit radionuclide materials. CAP88-PC version 1.0 was approved by the United States Environmental Protection Agency (EPA) for use in calculating Effective Dose Equivalent (EDE) values in accordance with Subpart $H$ of 40 CFR 61 . The DOE has since released CAP88-PC version 2.0, which has not yet been approved by the EPA. Version 2.0 provides several advantages over version 1.0.

In order to use version 2.0 when making Subpart $\mathrm{H}$ calculations approval to do so must be requested from the EPA. In support of any request for approval it must be demonstrated that the EDE values obtained via version 2.0 are comparable to the values obtained when using version 1.0. This report describes a comparison of the two CAP88-PC versions which shows that when using similar inputs, the two versions yield identical results. It is recommended that a request be made of the EPA to approve CAP88-PC version 2.0 for use in calculating EDE values in accordance with Subpart $H$ of 40 CFR 61.

This technical information was prepared as an account of work sponsored by an agency of the United States Government. Neither the United States Government or any agencies thereof, nor any of their employees, nor any of its contractors, subcontractors nor their employees make any warranty, express or implied, or usefulness of any information, apparatus, product, or process disclosed, or represents that its use would not infringe privately owned rights. Reference herein to any specific commercial product, process, or service by trade name, trademark, manufacturer, or otherwise, does not necessarily constitute or imply its endorsement, recommendation, or favoring by the United States Government or any agency thereof. The views and opinions of authors expressed herein do not necessarily state or reflect those of the United States Government or any thereof, of Fluor Daniel Fernald, its affiliates or its parent companies.

The submitted manuscript has been authored by a contractor (grantee) of the U.S. Government under contract (grant) No. DE-AC24-920R21972. Accordingly, the U.S. Government retains a non-exclusive, royalty-free license to publish or reproduce this contribution, or allow others to do so for the U.S. Government purposes.

Fluor Daniel Fornald with the U.S. Department of Energy, under Contract No. DE-AC24$920 R 21972$. 


\subsection{INTRODUCTION}

40 CFR Part 61 (Subpart $H$ of the NESHAP) requires DOE facilities to use approved sampling procedures, computer models, or other approved procedures when calculating Effective Dose Equivalent (EDE) values to members of the public. Currently version 1.0 of the approved computer model CAP88-PC is used to calculate EDE values. The DOE has upgraded the CAP88-PC software to version 2.0. This version provides simplified data entry, better printing characteristics, the use of a mouse, and other features.

The DOE has developed and released version 2.0 for testing and comment. This new software is a WINDOWS - based application that offers a new graphical user interface with new utilities for preparing and managing population and weather data, and several new decay chains. The program also allows the user to view results before printing. This document describes a test that confirmed CAP88-PC version 2.0 generates results comparable to the original version of the CAP88-PC program.

\subsection{EVALUATION PROCEDURE}

To confirm that the algorithms used in version 2.0 are identical to those used in version 1.0 three sets of input data were created to be run under both CAP88-PC versions. These datasets are summarized in Table 1.

Each of the three datasets were run under each of the CAP88-PC versions. For a given dataset, the resulting EDE values from each CAP88-PC version were compared. 


\begin{tabular}{|c|c|c|c|c|c|c|c|c|c|c|c|}
\hline \multirow[b]{2}{*}{ Nuclide } & \multirow[b]{2}{*}{ Class } & \multirow[b]{2}{*}{ Size } & \multirow{2}{*}{$\begin{array}{c}\begin{array}{c}\text { DATASET } \\
\# 1\end{array} \\
\begin{array}{l}\text { Source } \\
\# 1 \mathrm{Ci} / \mathrm{Y}\end{array}\end{array}$} & \multicolumn{2}{|c|}{$\begin{array}{c}\text { DATASET } \\
\# 2\end{array}$} & \multicolumn{6}{|c|}{$\begin{array}{l}\text { DATASET } \\
\# 3\end{array}$} \\
\hline & & & & $\begin{array}{l}\text { Source } \\
\# 1 \mathrm{Ci} / \mathrm{y}\end{array}$ & $\begin{array}{l}\text { Source } \\
\# 2 \mathrm{Ci} / \mathrm{Y}\end{array}$ & $\begin{array}{l}\text { Source } \\
\# 1 \mathrm{Ci} / \mathrm{y}\end{array}$ & $\begin{array}{l}\text { Source } \\
\# 2 \mathrm{Ci} / \mathrm{y}\end{array}$ & $\begin{array}{l}\text { Source } \\
\# 3 \mathrm{Ci} / \mathrm{y}\end{array}$ & $\begin{array}{l}\text { Source } \\
\# 4 \mathrm{Ci} / \mathrm{y}\end{array}$ & $\begin{array}{l}\text { Source } \\
\# 5 \mathrm{Ci} / \mathrm{y}\end{array}$ & $\begin{array}{l}\text { Source } \\
\# 6 \mathrm{Ci} / \mathrm{V}\end{array}$ \\
\hline Th-228 & $Y$ & 0.30 & 4.3E-07 & 4.3E-07 & 4.3E-07 & 4.3E-07 & $1.0 E+O O$ & $1.0 E+O 0$ & $1.0 E+00$ & $1.0 E+O O$ & $1.0 E+00$ \\
\hline Th-230 & $\mathbf{Y}$ & 0.30 & 1.3E-06 & $1.3 \mathrm{E}-06$ & $1.3 \mathrm{E}-06$ & $1.3 E-06$ & $1.0 E-05$ & $1.0 E+00$ & $1.0 E+\infty 0$ & $1.0 E+00$ & $1.0 E+00$ \\
\hline Th-232 & $Y$ & 0.30 & $6.8 E-08$ & $6.8 \mathrm{E}-08$ & $6.8 \mathrm{E}-08$ & $6.8 \mathrm{E}-08$ & $1.2 \mathrm{E}-05$ & $1.0 E+00$ & $1.0 E+00$ & $1.0 E+00$ & $1.0 E+00$ \\
\hline Th-234 & $\mathbf{Y}$ & 0.30 & 6.4E-05 & $6.4 E-05$ & $6.4 E-05$ & $6.4 \mathrm{E}-05$ & $1.0 E-05$ & $1.0 E+00$ & $1.0 E+00$ & $1.0 E+00$ & $1.0 E+O 0$ \\
\hline $\mathrm{H}-3$ & - & 1.00 & & & & $1.0 E-05$ & $1.0 \mathrm{E}-05$ & $1.0 E+00$ & $1.0 E+00$ & $1.0 E+00$ & $1.0 E+00$ \\
\hline $\mathrm{Np}-237$ & $w$ & 1.00 & & & & 1.0E-05 & $1.0 E-05$ & $1.0 \mathrm{E}+00$ & $1.0 E+00$ & $1.0 E+00$ & $1.0 E+00$ \\
\hline Np-238 & $w$ & 1.00 & & & & $1.0 \mathrm{E}-05$ & $1.0 \mathrm{E}-05$ & $1.0 E+00$ & $1.0 E+00$ & $1.0 E+00$ & $1.0 E+00$ \\
\hline Np-239 & $w$ & 1.00 & & & & $1.0 \mathrm{E}-05$ & $1.0 \mathrm{E}-05$ & $1.0 E+00$ & $1.0 E+00$ & $1.0 E+00$ & $1.0 E+00$ \\
\hline $\mathrm{Np}-240$ & $w$ & 1.00 & & & & 2.0E-05 & $1.0 \mathrm{E}-05$ & $1.0 E+O 0$ & $1.0 E+00$ & $1.0 E+00$ & $1.0 E+00$ \\
\hline $\mathrm{Np}-240 \mathrm{~m}$ & $w$ & 1.00 & & & & 3.0E-05 & $1.08-05$ & $1.0 E+00$ & $1.0 E+00$ & $1.0 E+00$ & $1.0 E+00$ \\
\hline U-234 & $Y$ & 0.30 & $1.4 E-05$ & $1.4 E-05$ & $1.4 \mathrm{E}-05$ & & & & & & \\
\hline $\mathrm{U}-235$ & $Y$ & 1.00 & $7.6 \mathrm{E}-07$ & $7.6 \mathrm{E}-07$ & 7.6E-07 & & & & & & \\
\hline U-236 & $Y$ & 1.00 & $5.6 \mathrm{E}-07$ & $5.6 \mathrm{E}-07$ & $5.6 \mathrm{E}-07$ & & & & & & \\
\hline$U-238$ & $Y$ & 0.30 & $1.6 \mathrm{E}-05$ & $1.6 \mathrm{E}-05$ & $1.6 \mathrm{E}-05$ & & & & & & \\
\hline RA-226 & $w$ & 0.30 & 1.0E-08 & $1.0 E-08$ & $1.0 E-08$ & & & & & & \\
\hline RA-228 & $w$ & 0.30 & 4.1E-08 & 4.1E-08 & 4.1E-08 & & & & & & \\
\hline \multicolumn{3}{|c|}{ Stack Height (m) } & 8.0 & 8.0 & 8.0 & 8.0 & 9.0 & 10.0 & 9.0 & 7.0 & 20.0 \\
\hline \multicolumn{3}{|c|}{ Diameter $(m)$} & 6.0 & 0.3 & 0.3 & 6.0 & 6.0 & 5.0 & 4.0 & 1.0 & 1.0 \\
\hline \multicolumn{3}{|c|}{$\begin{array}{l}\text { Plume Rise Momentum } \\
(\mathrm{m} / \mathrm{s})\end{array}$} & 123.0 & $1.0 E+00$ & $1.0 E+\infty O$ & 123 & 130 & 120 & 100 & 150 & 165 \\
\hline \multicolumn{3}{|c|}{ Temperature (degrees C) } & 9 & \multicolumn{2}{|c|}{9} & \multicolumn{6}{|c|}{9} \\
\hline \multicolumn{3}{|c|}{ Precipitation $(\mathrm{cm} / \mathrm{y})$} & 150 & \multicolumn{2}{|c|}{150} & \multicolumn{6}{|c|}{150} \\
\hline \multicolumn{3}{|c|}{ Mixing Height (m) } & 950 & \multicolumn{2}{|c|}{950} & \multicolumn{6}{|c|}{950} \\
\hline \multicolumn{3}{|l|}{ Wind File } & 1996.WND & \multicolumn{2}{|c|}{ 1996.WND } & \multicolumn{6}{|c|}{ ERIEPA.WND } \\
\hline \multicolumn{3}{|c|}{ Type of Run } & Individual & \multicolumn{2}{|c|}{ Individual } & \multicolumn{6}{|c|}{ Population } \\
\hline \multicolumn{3}{|c|}{ Population File } & None & \multicolumn{2}{|c|}{ None } & \multicolumn{6}{|c|}{ RMIASHTA.POP } \\
\hline \multicolumn{3}{|c|}{ Distances $(m)$} & $\begin{array}{c}1,2,3,4 \\
5 .\end{array}$ & \multicolumn{2}{|c|}{$\begin{array}{c}364,377,569,578 \\
751,880,896,972 \\
1004,1111,1239 \\
1243,1345,1416 \\
1553 .\end{array}$} & 310,810 & 1500,2500 & $\begin{array}{l}3500,450 \\
5000,5500\end{array}$ & $\begin{array}{l}0,7500,15 \\
00,70000 .\end{array}$ & 5000,2500 & 35000 \\
\hline
\end{tabular}

TABLE 1 - CAP88-PC INPUT DATASETS 
Table 2 summarizes the results for the Maximally Exposed Individual (MEl) for each of the CAP88-PC runs. This Table shows that, for a given input dataset, both versions of CAP88$P C$ yield identical EDE values for the MEl.

\begin{tabular}{|c|c|c|c|c|c|c|c|c|c|}
\hline & \multicolumn{3}{|c|}{ DATASET \#1 } & \multicolumn{3}{|c|}{ DATASET \#2 } & \multicolumn{3}{|c|}{ DATASET \#3 } \\
\hline & $\begin{array}{c}\text { Vorsion } \\
1.0\end{array}$ & $\begin{array}{l}\text { Version } \\
2.0\end{array}$ & Change & $\begin{array}{l}\text { Version } \\
1.0\end{array}$ & $\begin{array}{l}\text { Version } \\
2.0\end{array}$ & Change & $\begin{array}{l}\text { Version } \\
1.0\end{array}$ & $\begin{array}{l}\text { Version } \\
2.0\end{array}$ & Change \\
\hline $\begin{array}{l}\text { Effective Dose Equivalent } \\
\text { (mrem/yr) }\end{array}$ & $8.28 \theta-02$ & $8.28 \theta-02$ & $0 \%$ & $8.04 e-02$ & $8.040-02$ & $0 \%$ & $3.46 \theta-02$ & $3.46 \theta-02$ & $0 \%$ \\
\hline $\begin{array}{l}\text { Locetion of the Maximally } \\
\text { Exposed Individual }\end{array}$ & $\begin{array}{l}1 \text { Meter } \\
\text { Northeast }\end{array}$ & $\begin{array}{l}1 \text { Meter } \\
\text { Northeast }\end{array}$ & None & $\begin{array}{c}364 \\
\text { Meters } \\
\text { Northeast }\end{array}$ & $\begin{array}{c}364 \\
\text { Meters } \\
\text { Northeast }\end{array}$ & None & $\begin{array}{c}2500 \\
\text { Meters } \\
\text { North }\end{array}$ & $\begin{array}{l}2500 \\
\text { Meters } \\
\text { North }\end{array}$ & None \\
\hline Lifetime Fatal Cancer Risk & $4.56 e-07$ & $4.56 \theta-07$ & $0 \%$ & $1.04 \theta-06$ & $1.04 \theta-06$ & $0 \%$ & $3.24 \theta-03$ & $3.24 \theta-03$ & $0 \%$ \\
\hline
\end{tabular}

TABLE 2 - CAP88-PC MEI OUTPUT SUMMARY

The EDE values calculated by the CAP88-PC programs for other members of the public were also compared. Again, for a given dataset, no differences were found in the EDE values calculated between versions 1.0 and 2.0. Attachments $A, B$, and $C$ contain the Synopsis and Summary files for each dataset run under versions 1.0 and 2.0 .

The advantages of using version 2.0 come not from more accurate calculations, but from a more user-friendly program. Version 2.0 offers the convenience of choosing some program inputs via a mouse; it provides a better format of printouts; and, it saves files by their dataset names instead of sequenced letters.

With version 2.0, a user may set up the CAP88-PC program to accommodate his/her available printer. Version 1.0 does not have this ability and as a result printouts are formatted poorly and use more paper than necessary. To print the CAP88-PC outputs in an acceptable format, users must exit the program and use a word processing program to reformat the files. If multiple CAP88-PC runs are saved in the CAP88-PC output subdirectory, identification of the desired files is onerous. Files saved by their dataset names are much easier to locate than files saved through a sequenced letters system.

\subsection{CONCLUSIONS}

(1) For like inputs, CAP88-PC version 2.0 yields results identical to that of version 1.0.

(2) CAP88-PC version 2.0 is a more compatible program to our computer operating system.

\subsection{RECOMMENDATIONS}

(1) FDF should request EPA approval to use CAP88-PC version 2.0 for Subpart $H$ purposes.

(2) The FC\&DP Environmental Compliance Team should develop procedures to instruct team members on how to use CAP88-PC version 2.0. 
ATTACHMENT A - DATASET \#1,

CAP88-PC v1.0 and v2.0 SYNOPSIS and SUMMARY FILES 
$C A P 88-P C$

Version 1.00

Clean Air Act Assessment Package - 1988

S Y N O P S I S RE POR T

Non-Radon Individual Assessment

Dec 15, $1997 \quad 1: 13 \mathrm{pm}$

Facility: FERNALD ENVIRONMENTAL MANAGEMENT PROJECT

Address: P.O. BOX 398704

7400 WILLEY ROAD

City: CINCINNATI

State: $\mathrm{OH} \quad$ Zip: $45253-8704$ 


\title{
Effective Dose Equivalent (mrem/year)
}

\section{$8.28 E-02$}

\author{
At This Location: 1 Meters Northeast \\ Source Category: REMEDIATION SITE \\ Source Type: Stack \\ Emission Year: 1997
}

\author{
Comments: Dataset \#1. Individual \\ Evaluative Test Run with one source.
}

\author{
Dataset Name: Dataset \#1 \\ Dataset Date: Dec 15, $1997 \quad$ 1:13 pm \\ Wind File: WNDFILES 1996. WND
}


Dec $15,1997 \quad 1: 13 \mathrm{pm}$

SYNOPSIS

Page 1

MAXIMALLY EXPOSED INDIVIDUAL

Location of The Individual: 1 Meters Northeast

Lifetime Fatal Cancer Risk: 4.56E-07

ORGAN DOSE EQUIVALENT SUMMARY

\begin{tabular}{lc} 
Organ & $\begin{array}{c}\text { Dose } \\
\text { Equivalent } \\
\text { (mrem/y) }\end{array}$ \\
\cline { 2 - 2 } GONADS & $3.97 \mathrm{E}-03$ \\
BREAST & $4.59 \mathrm{E}-03$ \\
R MAR & $8.83 \mathrm{E}-02$ \\
LUNGS & $3.62 \mathrm{E}-03$ \\
THYROID & $3.85 \mathrm{E}-03$ \\
ENDOST & $1.25 \mathrm{E}+00$ \\
RMNDR & $1.09 \mathrm{E}-01$ \\
EFFEC & $8.28 \mathrm{E}-02$
\end{tabular}


Dec 15, $1997 \quad 1: 13 \mathrm{pm}$

:NOPSIS

Page 2

RADIONUCLIDE EMISSIONS DURING THE YEAR 1997

\begin{tabular}{|c|c|c|c|c|}
\hline$\because$ acl ide & Class & Size & $\begin{array}{c}\text { Source } \\
\# 1 \\
\mathrm{Ci} / \mathrm{y}\end{array}$ & $\begin{array}{l}\text { TOTAL } \\
\mathrm{Ci} / \mathrm{y}\end{array}$ \\
\hline $\begin{array}{l}H H-228 \\
T H-230 \\
T H-232 \\
T H-234 \\
U-234 \\
U-235 \\
-1-236 \\
H-238 \\
F A-226 \\
F A-228\end{array}$ & $\begin{array}{l}Y \\
Y \\
Y \\
Y \\
Y \\
Y \\
Y \\
Y \\
Y \\
W \\
W\end{array}$ & $\begin{array}{l}0.30 \\
0.30 \\
0.30 \\
0.30 \\
0.30 \\
1.00 \\
1.00 \\
0.30 \\
0.30 \\
0.30\end{array}$ & $\begin{array}{l}4.3 E-07 \\
1.3 E-06 \\
6.8 E-08 \\
6.4 E-05 \\
1.4 E-05 \\
7.6 E-07 \\
5.6 E-07 \\
1.6 E-05 \\
1.0 E-08 \\
4.1 E-08\end{array}$ & $\begin{array}{l}4.3 E-07 \\
1.3 E-06 \\
6.8 E-08 \\
6.4 E-05 \\
1.4 E-05 \\
7.6 E-07 \\
5.6 E-07 \\
1.6 E-05 \\
1.0 E-08 \\
4.1 E-08\end{array}$ \\
\hline
\end{tabular}

\section{SITE INFORMATION}

Temperature:

Precipitation:

Mixing Height:

9 degrees $\mathrm{C}$

$150 \mathrm{~cm} / \mathrm{y}$

$950 \mathrm{~m}$ 
Dec 15, $1997 \quad 1: 13$ pm SYNOPSIS

Page 3

SOURCE INFORMATION

\begin{tabular}{rr} 
Source Number: & 1 \\
\cline { 2 - 2 } & \\
Stack Height $(\mathrm{m}):$ & 8.00 \\
Diameter $(\mathrm{m}):$ & 6.00 \\
PTume Rise & \\
Momentum (m/s): & $1.23 E+02$ \\
(Exit Velocity) &
\end{tabular}

AGRICULTURAL DATA

\begin{tabular}{|c|c|c|}
\hline & Vegetable & Milk \\
\hline $\begin{array}{r}\text { Fraction Home Produced: } \\
\text { Fraction From Assessment Area: } \\
\text { Fraction Imported: }\end{array}$ & $\begin{array}{l}0.700 \\
0.300 \\
0.000\end{array}$ & $\begin{array}{l}0.399 \\
0.601 \\
0.000\end{array}$ \\
\hline
\end{tabular}

Food Arrays were not generated for this run. Default Values used.

DISTANCES USED FOR MAXIMUM INDIVIDUAL ASSESSMENT

$\begin{array}{lllll}1 & 2 & 3 & 4 & 5\end{array}$




$$
\begin{gathered}
\text { C A P } 88-P C \\
\text { Version } 1.00 \\
\text { Clean Air Act Assessment Package - } 1988
\end{gathered}
$$

DOSE AND RISK EQUIVALENT SUMMARIES Non-Radon Individual Assessment Dec 15, 1997 1:13 pm

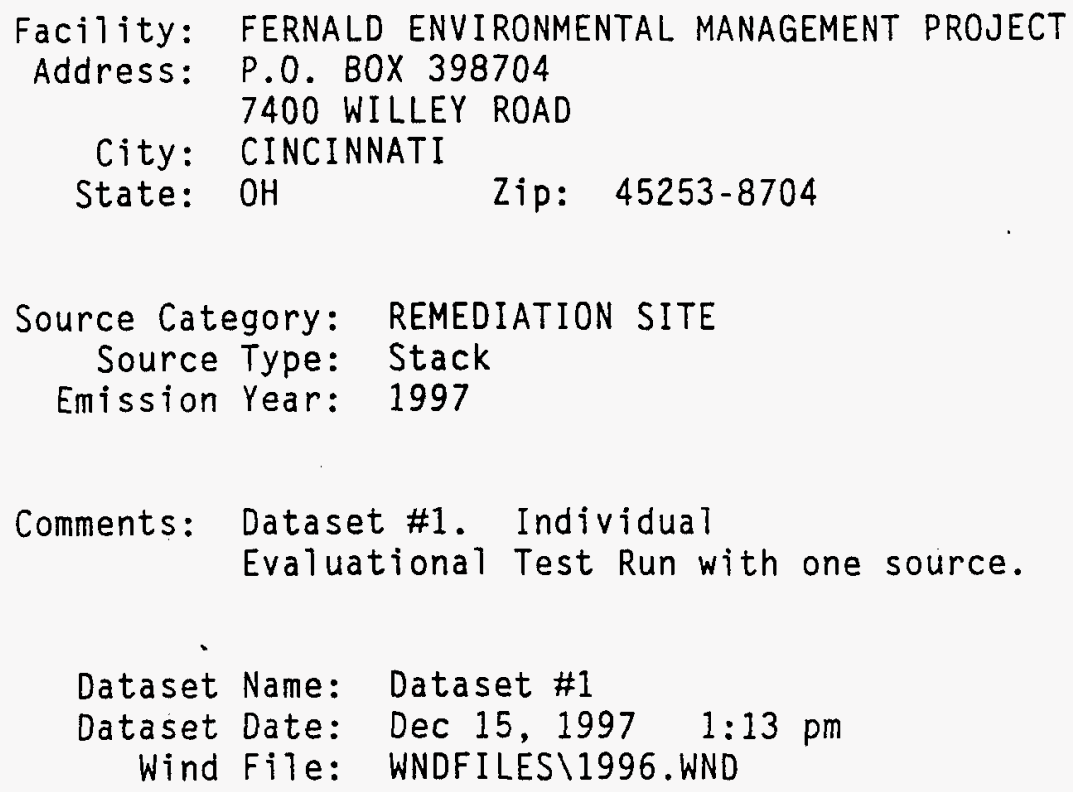


ORGAN DOSE EQUIVALENT SUMMARY

\begin{tabular}{lc} 
Organ & $\begin{array}{c}\text { Selected } \\
\text { Individual } \\
\text { (mrem/y) }\end{array}$ \\
\hline GONADS & $3.97 \mathrm{E}-03$ \\
BREAST & $4.59 \mathrm{E}-03$ \\
R MAR & $8.83 \mathrm{E}-02$ \\
LUNGS & $3.62 \mathrm{E}-03$ \\
THYROID & $3.85 \mathrm{E}-03$ \\
ENDOST & $1.25 \mathrm{E}+00$ \\
RMNDR & $1.09 \mathrm{E}-01$ \\
EFFEC & $8.28 \mathrm{E}-02$
\end{tabular}

PATHWAY EFFECTIVE DOSE EQUIVALENT SUMMARY

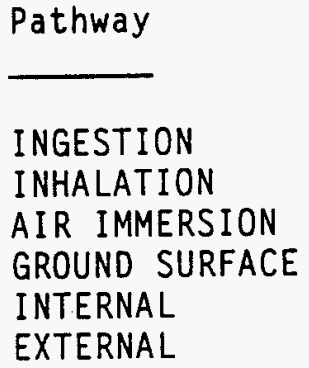

TOTAL
Selected

Individua 1

(mrem/y)

8.19E-02

$0.00 E+00$

$0.00 E+00$

9.04E- 04

8.19E-02

9.04E-04

$8.28 E-02$ 
NUCLIDE EFFECTIVE DOSE EQUIVALENT SUMMARY

Nuclide

TH-228

$\mathrm{TH}-230$

TH-232

TH-234

$\mathrm{U}-234$

$U-235$

$\mathrm{U}-236$

$\mathrm{U}-238$

$\mathrm{RA}-226$

$R A-228$

TOTAL
Selected Individua 7

(mrem/y)

$1.78 E-04$

$1.54 \mathrm{E}-03$

$7.34 E-05$

$9.51 \mathrm{E}-04$

$3.74 E-02$

$2.69 \mathrm{E}-03$

1. $42 \mathrm{E}-03$

$3.85 E-02$

$3.23 \mathrm{E}-05$

$6.74 E-05$

8. 28E-02 


\section{CANCER RISK SUMMARY}

\begin{tabular}{lc} 
Cancer & $\begin{array}{c}\text { Selected Individual } \\
\text { Total Lifetime } \\
\text { Fatal Cancer Risk }\end{array}$ \\
\cline { 2 - 2 } LEUKEMIA & $9.98 \mathrm{E}-08$ \\
BONE & $6.64 \mathrm{E}-08$ \\
THYROID & $9.24 \mathrm{E}-10$ \\
BREAST & $1.07 \mathrm{E}-08$ \\
LUNG & $8.82 \mathrm{E}-09$ \\
STOMACH & $6.75 \mathrm{E}-09$ \\
BOWEL & $1.70 \mathrm{E}-08$ \\
LIVER & $5.79 \mathrm{E}-09$ \\
PANCREAS & $3.96 \mathrm{E}-09$ \\
URINARY & $2.30 \mathrm{E}-07$ \\
OTHER & $4.84 \mathrm{E}-09$ \\
TOTAL & $4.56 \mathrm{E}-07$
\end{tabular}

PATHWAY RISK SUMMARY

\section{Pathway \\ INGESTION INHALATION \\ AIR IMMERSION GROUND SURFACE INTERNAL EXTERNAL}

TOTAL
Selected Individual Total Lifetime Fatal Cancer Risk

$$
4.35 \mathrm{E}-07
$$$$
0.00 E+00
$$$$
0.00 E+00
$$$$
2.08 E-08
$$$$
4.35 \mathrm{E}-07
$$

$2.08 E-08$

4.56E- 07 


\section{NUCLIDE RISK SUMMARY}

Nuclide

TH -228

$\mathrm{TH}-230$

$\mathrm{TH}-232$

$\mathrm{TH}-234$

$U-234$

$U-235$

$U-236$

$U-238$

$\mathrm{RA}-226$

$R A-228$

TOTAL
Selected Individual

Total Lifetime Fatal Cancer Risk

8. $54 \mathrm{E}-10$

$5.00 E-09$

2.34E-10

$1.08 E-08$

$1.89 \mathrm{E}-07$

2.77E-08

$7.16 \mathrm{E}-09$

$2.14 \mathrm{E}-07$

1. $71 E-10$

$4.80 E-10$

$4.56 E-07$ 
INDIVIDUAL EFFECTIVE DOSE EQUIVALENT RATE (mrem/y)

(AIl Radionuclides and Pathways)

\begin{tabular}{|c|c|c|c|c|c|}
\hline \multirow[b]{2}{*}{ Direction } & \multicolumn{5}{|c|}{ Distance (m) } \\
\hline & 1 & 2 & 3 & 4 & 5 \\
\hline $\begin{array}{r}N \\
N N W \\
N W \\
W N W \\
W \\
W S W \\
S W \\
S S W \\
S \\
S S E \\
S E \\
E S E \\
E \\
E N E \\
N E \\
N N E\end{array}$ & $\begin{array}{l}4.8 \mathrm{E}-02 \\
3.5 \mathrm{E}-02 \\
3.6 \mathrm{E}-02 \\
3.4 \mathrm{E}-02 \\
5.1 \mathrm{E}-02 \\
5.5 \mathrm{E}-02 \\
3.3 \mathrm{E}-02 \\
3.0 \mathrm{E}-02 \\
3.2 \mathrm{E}-02 \\
4.5 \mathrm{E}-02 \\
5.6 \mathrm{E}-02 \\
5.5 \mathrm{E}-02 \\
6.6 \mathrm{E}-02 \\
6.7 \mathrm{E}-02 \\
8.3 \mathrm{E}-02 \\
6.5 \mathrm{E}-02\end{array}$ & $\begin{array}{l}2.9 \mathrm{E}-02 \\
2.2 \mathrm{E}-02 \\
2.2 \mathrm{E}-02 \\
2.2 \mathrm{E}-02 \\
3.0 \mathrm{E}-02 \\
3.2 \mathrm{E}-02 \\
2.1 \mathrm{E}-02 \\
2.0 \mathrm{E}-02 \\
2.1 \mathrm{E}-02 \\
2.7 \mathrm{E}-02 \\
3.2 \mathrm{E}-02 \\
3.2 \mathrm{E}-02 \\
3.8 \mathrm{E}-02 \\
3.9 \mathrm{E}-02 \\
4.6 \mathrm{E}-02 \\
3.8 \mathrm{E}-02\end{array}$ & $\begin{array}{l}2.2 \mathrm{E}-02 \\
1.8 \mathrm{E}-02 \\
1.8 \mathrm{E}-02 \\
1.7 \mathrm{E}-02 \\
2.3 \mathrm{E}-02 \\
2.4 \mathrm{E}-02 \\
1.7 \mathrm{E}-02 \\
1.5 \mathrm{E}-02 \\
1.7 \mathrm{E}-02 \\
2.1 \mathrm{E}-02 \\
2.5 \mathrm{E}-02 \\
2.5 \mathrm{E}-02 \\
2.9 \mathrm{E}-02 \\
2.9 \mathrm{E}-02 \\
3.3 \mathrm{E}-02 \\
2.8 \mathrm{E}-02\end{array}$ & $\begin{array}{l}1.9 \mathrm{E}-02 \\
1.6 \mathrm{E}-02 \\
1.6 \mathrm{E}-02 \\
1.5 \mathrm{E}-02 \\
1.9 \mathrm{E}-02 \\
2.0 \mathrm{E}-02 \\
1.5 \mathrm{E}-02 \\
1.4 \mathrm{E}-02 \\
1.5 \mathrm{E}-02 \\
1.8 \mathrm{E}-02 \\
2.1 \mathrm{E}-02 \\
2.1 \mathrm{E}-02 \\
2.4 \mathrm{E}-02 \\
2.4 \mathrm{E}-02 \\
2.7 \mathrm{E}-02 \\
2.4 \mathrm{E}-02\end{array}$ & $\begin{array}{l}1.7 \mathrm{E}-02 \\
1.4 \mathrm{E}-02 \\
1.4 \mathrm{E}-02 \\
1.4 \mathrm{E}-02 \\
1.7 \mathrm{E}-02 \\
1.8 \mathrm{E}-02 \\
1.4 \mathrm{E}-02 \\
1.3 \mathrm{E}-02 \\
1.4 \mathrm{E}-02 \\
1.6 \mathrm{E}-02 \\
1.8 \mathrm{E}-02 \\
1.9 \mathrm{E}-02 \\
2.1 \mathrm{E}-02 \\
2.1 \mathrm{E}-02 \\
2.4 \mathrm{E}-02 \\
2.1 \mathrm{E}-02\end{array}$ \\
\hline
\end{tabular}


INDIVIDUAL LIFETIME RISK (deaths)

(All Radionuclides and Pathways)

\begin{tabular}{|c|c|c|c|c|c|}
\hline \multirow[b]{2}{*}{ Direction } & \multicolumn{5}{|c|}{ Distance (m) } \\
\hline & 1 & 2 & 3 & 4 & 5 \\
\hline $\begin{array}{r}N \\
N N W \\
N W \\
W N W \\
W \\
W S W \\
S W \\
S S W \\
S \\
S S E \\
S E \\
E S E \\
E \\
E N E \\
N E \\
N N E\end{array}$ & $\begin{array}{l}2.6 E-07 \\
1.9 E-07 \\
2.0 E-07 \\
1.9 E-07 \\
2.8 E-07 \\
3.0 E-07 \\
1.8 E-07 \\
1.6 E-07 \\
1.8 E-07 \\
2.5 E-07 \\
3.1 E-07 \\
3.0 E-07 \\
3.6 E-07 \\
3.7 E-07 \\
4.6 E-07 \\
3.6 E-07\end{array}$ & $\begin{array}{l}1.6 \mathrm{E}-07 \\
1.2 \mathrm{E}-07 \\
1.2 \mathrm{E}-07 \\
1.2 \mathrm{E}-07 \\
1.6 \mathrm{E}-07 \\
1.7 \mathrm{E}-07 \\
1.2 \mathrm{E}-07 \\
1.1 \mathrm{E}-07 \\
1.1 \mathrm{E}-07 \\
1.5 \mathrm{E}-07 \\
1.8 \mathrm{E}-07 \\
1.8 \mathrm{E}-07 \\
2.1 \mathrm{E}-07 \\
2.1 \mathrm{E}-07 \\
2.5 \mathrm{E}-07 \\
2.1 \mathrm{E}-07\end{array}$ & $\begin{array}{l}1.2 \mathrm{E}-07 \\
9.6 \mathrm{E}-08 \\
9.7 \mathrm{E}-08 \\
9.4 \mathrm{E}-08 \\
1.2 \mathrm{E}-07 \\
1.3 \mathrm{E}-07 \\
9.4 \mathrm{E}-08 \\
8.7 \mathrm{E}-08 \\
9.2 \mathrm{E}-08 \\
1.1 \mathrm{E}-07 \\
1.3 \mathrm{E}-07 \\
1.4 \mathrm{E}-07 \\
1.6 \mathrm{E}-07 \\
1.6 \mathrm{E}-07 \\
1.8 \mathrm{E}-07 \\
1.5 \mathrm{E}-07\end{array}$ & $\begin{array}{l}1.0 \mathrm{E}-07 \\
8.4 \mathrm{E}-08 \\
8.4 \mathrm{E}-08 \\
8.2 \mathrm{E}-08 \\
1.0 \mathrm{E}-07 \\
1.1 \mathrm{E}-07 \\
8.3 \mathrm{E}-08 \\
7.7 \mathrm{E}-08 \\
8.1 \mathrm{E}-08 \\
9.7 \mathrm{E}-08 \\
1.1 \mathrm{E}-07 \\
1.1 \mathrm{E}-07 \\
1.3 \mathrm{E}-07 \\
1.3 \mathrm{E}-07 \\
1.5 \mathrm{E}-07 \\
1.3 \mathrm{E}-07\end{array}$ & $\begin{array}{l}9.2 \mathrm{E}-08 \\
7.7 \mathrm{E}-08 \\
7.7 \mathrm{E}-08 \\
7.5 \mathrm{E}-08 \\
9.3 \mathrm{E}-08 \\
9.8 \mathrm{E}-08 \\
7.6 \mathrm{E}-08 \\
7.1 \mathrm{E}-08 \\
7.4 \mathrm{E}-08 \\
8.7 \mathrm{E}-08 \\
9.9 \mathrm{E}-08 \\
1.0 \mathrm{E}-07 \\
1.1 \mathrm{E}-07 \\
1.2 \mathrm{E}-07 \\
1.3 \mathrm{E}-07 \\
1.1 \mathrm{E}-07\end{array}$ \\
\hline
\end{tabular}




$$
\begin{aligned}
& \text { C A P } 88-\text { P C } \\
& \text { Version } 2.00
\end{aligned}
$$

Clean Air Act Assessment Package - 1988

$$
\begin{gathered}
\text { S Y N O P S I S R E P O R T } \\
\text { Non-Radon Individual Assessment } \\
\text { Dec 16, } 199702: 25 \mathrm{pm}
\end{gathered}
$$

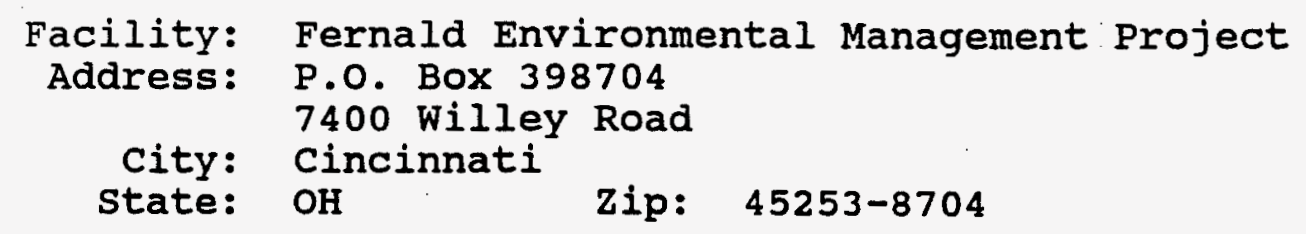

Source Category: Remediation site Source Type: Stack

Emission Year: 1997

Comments: Dataset \#1. Individual

Evaluative Test Run with one source

Effective Dose Equivalent

(mrem/year)

8. 28E-02

At This Location: 1 Meters Northeast

Dataset Name: Dataset12

Dataset Date: Dec 16, $199702: 24$ pm

Wind File: F:\CAP88PC2\WNDFILES $\backslash 1996$.WND 
iec $16,199702: 25 \mathrm{pm}$

MAXIMALLY EXPOSED INDIVIDUAL

Location of The Individual:

Lifetime Fatal Cancer Risk:

1 Meters Northeast

$4.56 \mathrm{E}-07$

ORGAN DOSE EQUIVALENT SUMMARY

\begin{tabular}{lc} 
Organ & $\begin{array}{c}\text { Dose } \\
\text { Equivalent } \\
(\mathrm{mrem} / \mathrm{Y})\end{array}$ \\
\hline GONADS & $3.97 \mathrm{E}-03$ \\
BREAST & $4.59 \mathrm{E}-03$ \\
R MAR & $8.83 \mathrm{E}-02$ \\
LUNGS & $3.62 \mathrm{E}-03$ \\
THYROID & $3.85 \mathrm{E}-03$ \\
ENDOST & $1.25 \mathrm{E}+00$ \\
RMNDR & $1.09 \mathrm{E}-01$ \\
EFFEC & $8.28 \mathrm{E}-02$
\end{tabular}


RADIONUCLIDE EMISSIONS DURING THE YEAR 1997

\begin{tabular}{|c|c|c|c|c|}
\hline Muc ide & Class & size & $\begin{array}{c}\text { Source } \\
\# 1 \\
c i / y\end{array}$ & $\begin{array}{r}\text { TOTAL } \\
\mathrm{Ci} / \mathrm{Y}\end{array}$ \\
\hline $\mathrm{TH}-228$ & $Y$ & 0.30 & $4.3 E-07$ & $4.3 E-07$ \\
\hline $\mathrm{TH}-230$ & $Y$ & 0.30 & $1.3 E-06$ & $1.3 E-06$ \\
\hline $\mathrm{TH}-232$ & $Y$ & 0.30 & $6.8 \mathrm{E}-08$ & $6.8 E-08$ \\
\hline $\mathrm{TH}-2.34$ & $Y$ & 0.30 & $6.4 \mathrm{E}-05$ & $6.4 E-05$ \\
\hline$U-23 t$ & $\bar{Y}$ & 0.30 & $1.4 \mathrm{E}-05$ & $1.4 E-05$ \\
\hline$U-23 j$ & $\mathrm{Y}$ & 1.00 & $7.6 \mathrm{E}-07$ & $7.6 \mathrm{E}-07$ \\
\hline$U-236$ & $\mathrm{Y}$ & 1.00 & $5.6 \mathrm{E}-07$ & $5.6 E-07$ \\
\hline$(I-2) 3$ & $Y$ & 0.30 & $1.6 \mathrm{E}-05$ & $1.6 E-05$ \\
\hline$R A-\ddot{2} \geq 6$ & W & 0.30 & 1. $0 \mathrm{E}-08$ & 1. $0 E-08$ \\
\hline$R A-228$ & $\mathrm{~W}$ & 0.30 & $4.1 E-08$ & $4.1 E-08$ \\
\hline
\end{tabular}

SITE INFORMATION

Temperature: Precipitation: Mixing Height: 
leec 15, $199702: 25 \mathrm{pm}$

\title{
SOURCE INFORMATION
}

\author{
Source Number: 1 \\ stack Height (m): \\ Diameter $(m)$ : \\ 8. \\ 6. \\ Plume Rise \\ Momentum (m/s): \\ (Exit Velocity) \\ 123 .
}

AGRICULTURAL DATA

$\begin{array}{ccccc} & \text { Vegetable } & \text { Milk } & \text { Meat } \\ \text { Fraction Home Produced: } & -0.700 & & -399 & 0.442 \\ \text { Fraction From Assessment Area: } & 0.300 & 0.601 & 0.558 \\ \text { Fraction Imported: } & 0.000 & 0.000 & 0.000\end{array}$

Food Arrays were not generated for this run. Default Values used.

DISTANCES (M) USED FOR MAXIMUM INDIVIDUAL ASSESSMENT

1

2

3

4

5 
C A P 8 \& - P C

Version 2.00

Clean Air Act Assessment Package - 1988

() O S E A N D R I S K E Q U I V A L E N T S U M M A R I E S Non-Radon Individual Assessment Dec 16, $199702: 25 \mathrm{pm}$

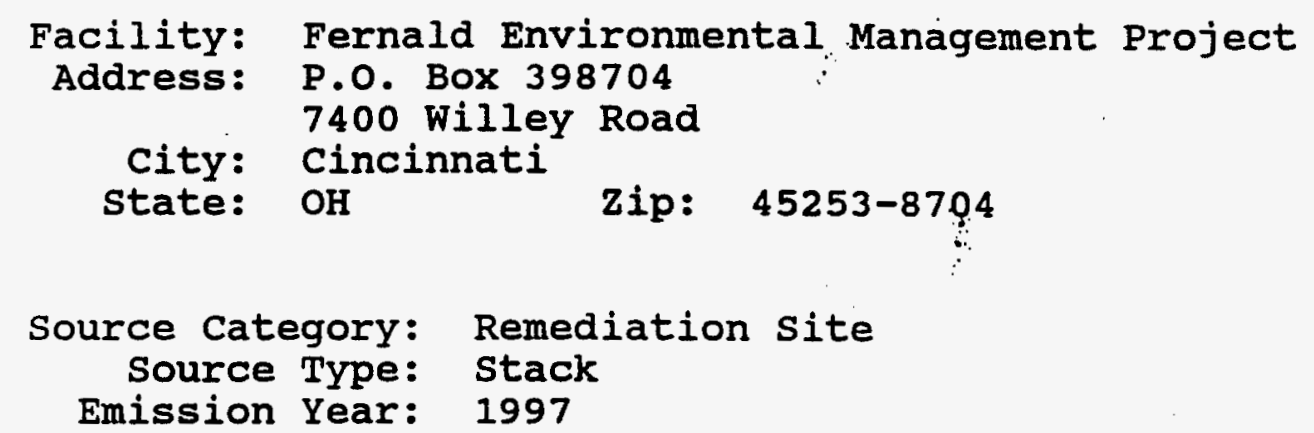

Comments: Dataset \#1. Individual

Evaluative Test Run with one source

Dataset Name: Dataset12

Dataset Date: Dec 16, 1997 02:24 pm

Wind File: F: \CAP88PC2 \WNDFILES \1996.WND 
ORGAN DOSE EQUIVALENT SUMMARY

\begin{tabular}{lc} 
Organ & $\begin{array}{c}\text { Selected } \\
\text { Individual } \\
\text { (mrem/Y) }\end{array}$ \\
\hline GONADS & $3.97 \mathrm{E}-03$ \\
BREAST & $4.59 \mathrm{E}-03$ \\
R MAR & $8.83 \mathrm{E}-02$ \\
LUNGS & $3.62 \mathrm{E}-03$ \\
THYROID & $3.85 \mathrm{E}-03$ \\
ENDOST & $1.25 \mathrm{E}+00$ \\
RMNDR & $1.09 \mathrm{E}-01$ \\
EFFEC & $8.28 \mathrm{E}-02$
\end{tabular}

PATHWAY EFFECTIVE DOSE EQUIVALENT SUMMARY

Selected

Individual

Pathway

(mrem/y)

INGESTION

8. 19E-02

INHALATION

$0.00 \mathrm{E}+00$

AIR IMMERSION

$0.00 \mathrm{E}+00$

GROUND SURFACE

$9.04 \mathrm{E}-04$

INTERNAL

EXTERNAL

8. 19E-02

$9.04 \mathrm{E}-04$

TOTAL

$8 \cdot 28 E-02$ 
NUCLIDE EFFECTIVE DOSE EQUIVALENT SUMMARY

Nuclide

TH-228

TH -230

TH-232

TH-234

U-234

$U-235$

$\mathrm{U}-236$

$\mathrm{U}-238$

RA-226

RA-228

TOTAL selected

Individual

(mrem/Y)

1. $78 \mathrm{E}-04$

1. $54 \mathrm{E}-03$

$7.34 \mathrm{E}-05$

9. $51 \mathrm{E}-04$

3. $.74 \mathrm{E}-02$

2. $69 \mathrm{E}-03$

1. $42 \mathrm{E}-03$

3. 85E-02

3. $23 E-05$

.6. $74 \mathrm{E}-05$

8. 28E-02 
CANCER RISK SUMMARY

\begin{tabular}{lc} 
& $\begin{array}{c}\text { Selected Individual } \\
\text { Total Lifetime } \\
\text { Fatal Cancer Risk }\end{array}$ \\
\hline Cancer & $9.98 \mathrm{E}-08$ \\
BEUKEMIA & $6.64 \mathrm{E}-08$ \\
THYROID & $9.24 \mathrm{E}-10$ \\
BREAST & $1.07 \mathrm{E}-08$ \\
LUNG & $8.82 \mathrm{E}-09$ \\
STOMACH & $6.75 \mathrm{E}-09$ \\
BOWEL & $1.70 \mathrm{E}-08$ \\
LIVER & $5.79 \mathrm{E}-09$ \\
PANCREAS & $3.96 \mathrm{E}-09$ \\
URINARY & $2.30 \mathrm{E}-07$ \\
OTHER & $4.84 \mathrm{E}-09$ \\
TOTAL & $4.56 \mathrm{E}-07$
\end{tabular}

PATHWAY RISK SUMMARY

Pathway

INGESTION

INHALATION

AIR IMMERSION GROUND SURFACE

INTERNAL

EXTERNAL

TOTAL
Selected Individual Total Lifetime Fatal Cancer Risk

4. 35E-07

$0.00 \mathrm{E}+00$

$0.00 \mathrm{E}+00$

2. $08 \mathrm{E}-08$

$4.35 E-07$

$2.08 \mathrm{E}-08$

$4.56 \mathrm{E}-07$ 


\section{NUCLIDE RISK SUMMARY}

Nuclide

TH-228

$\mathrm{TH}-230$

TH-232

TH -234

$\mathrm{U}-234$

$\mathrm{U}-235$

$\mathrm{U}-236$

$\mathrm{U}-238$

RA-226

$\mathrm{RA}-228$

TOTAL
Selected Individual Total Lifetime Fatal Cancer Risk
$8.54 \mathrm{E}-10$
5. 00E-09
2. $34 \mathrm{E}-10$
1. $08 \mathrm{E}-08$
1. $89 \mathrm{E}-07$
$2.77 \mathrm{E}-08$
7. $16 \mathrm{E}-09$
$2.14 \mathrm{E}-07$
$1.71 \mathrm{E}-10$
$4.80 \mathrm{E}-10$
4. 56E-07 
INDIVIDUAL EFFECTIVE DOSE EQUIVALENT RATE (mrem/Y) (All Radionuclides and Pathways)

Distance (m)

Direction

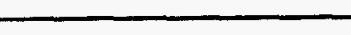

12

$\begin{array}{llll}3 & 4 & \because & 5\end{array}$

\begin{tabular}{|c|c|c|c|c|c|}
\hline $\mathrm{N}$ & $4.8 E-02$ & $2.9 \mathrm{E}-02$ & $2.2 E-02$ & 1.9E-02 & 1. $7 \mathrm{E}-02$ \\
\hline NNW & $3.5 E-02$ & $2.2 E-02$ & $1.8 \mathrm{E}-02$ & 1. $6 \mathrm{E}-02$ & $1.4 \mathrm{E}-02$ \\
\hline NW & $3.6 \mathrm{E}-02$ & $2.2 \mathrm{E}-02$ & $1.8 \mathrm{E}-02$ & $1.6 E-02$ & $1.4 \mathrm{E}-02$ \\
\hline WNW & $3.4 \mathrm{E}-02$ & $2.2 \mathrm{E}-02$ & $1.7 \mathrm{E}-02$ & $1.5 E-02$ & $1.4 \mathrm{E}-02$ \\
\hline$W$ & $5.1 E-02$ & 3. $0 \mathrm{E}-02$ & $2.3 E-02$ & $1.9 \mathrm{E}-02$ & $1.7 \mathrm{E}-02$ \\
\hline WSW & $5.5 E-02$ & $3.2 E-02$ & $2.4 E-02$ & 2. $0 \mathrm{E}-02$ & $1.8 \mathrm{E}-02$ \\
\hline SW & $3.3 E-02$ & $2.1 E-02$ & $1.7 E-02$ & $1.5 \mathrm{E}-02$ & $1.4 \mathrm{E}-02$ \\
\hline SSW & $3.0 E-02$ & $2.0 E-02$ & $1.6 E-02$ & $1.4 \mathrm{E}-02$ & $1.3 E-02$ \\
\hline$S$ & $3.2 E-02$ & $2.1 \mathrm{E}-02$ & $1.7 \mathrm{E}-02$ & $1.5 \mathrm{E}-02$ & $1.4 \mathrm{E}-02$ \\
\hline SSE & $4.5 E-02$ & $2.7 E-02$ & $2.1 E-02$ & $1.8 E-02$ & $1.6 E-02$ \\
\hline$S E$ & $5.6 \mathrm{E}-02$ & $3.2 \mathrm{E}-02$ & $2.5 E-02$ & $2.1 E-02$ & $1.8 E-02$ \\
\hline ESE & $5.5 \mathrm{E}-02$ & $3.2 E-02$ & $2.5 E-02$ & 2. $1 \mathrm{E}-02$ & $1.9 E-02$ \\
\hline$E$ & $6.6 E-02$ & $3.8 E-02$ & $2.9 \mathrm{E}-02$ & $2.4 \mathrm{E}-02$ & 2. $1 E-02$ \\
\hline ENE & $6.7 E-02$ & $3.9 E-02$ & $2.9 E-02$ & $2.4 \mathrm{E}-02$ & $2.1 E-02$ \\
\hline$N E$ & $8.3 E-02$ & $4.6 E-02$ & $3.3 E-02$ & 2. $7 \mathrm{E}-02$ & $2.4 \mathrm{E}-02$ \\
\hline NNF & $6.5 E-02$ & $3.8 E-02$ & $2.8 E-02$ & $2.4 \mathrm{E}-02$ & $2.1 E-02$ \\
\hline
\end{tabular}


INDIVIDUAL LIFETIME RISK (deaths)

(All Radionuclides and Pathways)

\section{Distance (m)}

\section{Direction}

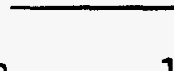

12

3

4

5

\begin{tabular}{rlllll}
\hline$N$ & $2.6 \mathrm{E}-07$ & $1.6 \mathrm{E}-07$ & $1.2 \mathrm{E}-07$ & $1.0 \mathrm{E}-07$ & $9.2 \mathrm{E}-08$ \\
$\mathrm{~N} W$ & $1.9 \mathrm{E}-07$ & $1.2 \mathrm{E}-07$ & $9.6 \mathrm{E}-08$ & $8.4 \mathrm{E}-08$ & $7.7 \mathrm{E}-08$ \\
$N W$ & $2.0 \mathrm{E}-07$ & $1.2 \mathrm{E}-07$ & $9.7 \mathrm{E}-08$ & $8.4 \mathrm{E}-08$ & $7.7 \mathrm{E}-08$ \\
$\mathrm{WNW}$ & $1.9 \mathrm{E}-07$ & $1.2 \mathrm{E}-07$ & $9.4 \mathrm{E}-08$ & $8.2 \mathrm{E}-08$ & $7.5 \mathrm{E}-08$ \\
$\mathrm{~W}$ & $2.8 \mathrm{E}-07$ & $1.6 \mathrm{E}-07$ & $1.2 \mathrm{E}-07$ & $1.0 \mathrm{E}-07$ & $9.3 \mathrm{E}-08$ \\
$\mathrm{WSW}$ & $3.0 \mathrm{E}-07$ & $1.7 \mathrm{E}-07$ & $1.3 \mathrm{E}-07$ & $1.1 \mathrm{E}-07$ & $9.8 \mathrm{E}-08$ \\
$\mathrm{SW}$ & $1.8 \mathrm{E}-07$ & $1.2 \mathrm{E}-07$ & $9.4 \mathrm{E}-08$ & $8.3 \mathrm{E}-08$ & $7.6 \mathrm{E}-08$ \\
$\mathrm{SSW}$ & $1.6 \mathrm{E}-07$ & $1.1 \mathrm{E}-07$ & $8.7 \mathrm{E}-08$ & $7.7 \mathrm{E}-08$ & $7.1 \mathrm{E}-08$ \\
$\mathrm{~S}$ & $1.8 \mathrm{E}-07$ & $1.1 \mathrm{E}-07$ & $9.2 \mathrm{E}-08$ & $8.1 \mathrm{E}-08$ & $7.4 \mathrm{E}-08$ \\
$\mathrm{SSE}$ & $2.5 \mathrm{E}-07$ & $1.5 \mathrm{E}-07$ & $1.1 \mathrm{E}-07$ & $9.7 \mathrm{E}-08$ & $8.7 \mathrm{E}-08$ \\
$\mathrm{SE}$ & $3.1 \mathrm{E}-07$ & $1.8 \mathrm{E}-07$ & $1.3 \mathrm{E}-07$ & $1.1 \mathrm{E}-07$ & $9.9 \mathrm{E}-08$ \\
$\mathrm{ESE}$ & $3.0 \mathrm{E}-07$ & $1.8 \mathrm{E}-07$ & $1.4 \mathrm{E}-07$ & $1.1 \mathrm{E}-07$ & $1.0 \mathrm{E}-07$ \\
$\mathrm{E}$ & $3.6 \mathrm{E}-07$ & $2.1 \mathrm{E}-07$ & $1.6 \mathrm{E}-07$ & $1.3 \mathrm{E}-07$ & $1.1 \mathrm{E}-07$ \\
$\mathrm{ENE}$ & $3.7 \mathrm{E}-07$ & $2.1 \mathrm{E}-07$ & $1.6 \mathrm{E}-07$ & $1.3 \mathrm{E}-07$ & $1.2 \mathrm{E}-07$ \\
$N E$ & $4.6 \mathrm{E}-07$ & $2.5 \mathrm{E}-07$ & $1.8 \mathrm{E}-07$ & $1.5 \mathrm{E}-07$ & $1.3 \mathrm{E}-07$ \\
$\mathrm{NNE}$ & $3.6 \mathrm{E}-07$ & $2.1 \mathrm{E}-07$ & $1.5 \mathrm{E}-07$ & $1.3 \mathrm{E}-07$ & $1.1 \mathrm{E}-07$ \\
& & & & & \\
\hline
\end{tabular}


ATTACHMENT A - DATASET \#2,

\author{
CAP88-PC v1.0 and v2.0 \\ SYNOPSIS and SUMMARY FILES
}


$C A P 88-P C$

Version 1.00

Clean Air Act Assessment Package - 1988

SYNOP S I S REPORT

Non-Radon Individual Assessment

Dec 15,1997 . 12:38 am

Facility: FERNALD ENVIRONMENTAL MANAGEMENT PROJECT

Address: P.O. BOX 398704

7400 WILLEY ROAD

City: CINCINNATI

State: $\mathrm{OH} \quad$ Zip: $45253-8704$ 


\begin{tabular}{c}
$\begin{array}{c}\text { Effective Dose Equivalent } \\
\text { (mrem/year) }\end{array}$ \\
\hline $8.04 E-02$ \\
\hline
\end{tabular}

At This Location: 364 Meters Northeast

Source Category: REMEDIATION SITE

Source Type: Stack

Emission Year: 1997

Comments: Dataset \#2. Individual
Evaluative Test Run with two sources.

Dataset Name: Dataset \#2

Dataset Date: Dec 15, 1997 12:38 am

Wind File: WNDFILES 11996. WND 
Dec 15, 1997 12:38 am

SYNOPSIS

Page 1

MAXIMALLY EXPOSED INDIVIDUAL

Location of The Individual: 364 Meters Northeast

Lifetime Fatal Cancer Risk: 1.04E-06

ORGAN DOSE EQUIVALENT SUMMARY

\begin{tabular}{lc} 
Organ & $\begin{array}{c}\text { Dose } \\
\text { Equivalent } \\
\text { (mrem/y) }\end{array}$ \\
\hline GONADS & $1.54 E-04$ \\
BREAST & $1.74 E-04$ \\
R MAR & $8.89 E-03$ \\
LUNGS & $6.22 E-01$ \\
THYROID & $1.50 E-04$ \\
ENDOST & $1.15 E-01$ \\
RMNDR & $3.71 E-03$ \\
EFFEC & $8.04 E-02$
\end{tabular}


Dec 15, 1997 12:38 am SYNOPSIS

RADIONUCLIDE EMISSIONS DURING THE YEAR 1997

\begin{tabular}{|c|c|c|c|c|c|}
\hline Nuclide & Class & Size & $\begin{array}{c}\text { Source } \\
\# 1 \\
\mathrm{Ci} / \mathrm{y}\end{array}$ & $\begin{array}{c}\text { Source } \\
\# 2 \\
\mathrm{Ci} / \mathrm{y}\end{array}$ & $\begin{array}{l}\text { TOTAL } \\
\mathrm{Ci} / \mathrm{y}\end{array}$ \\
\hline $\begin{array}{l}\text { TH-228 } \\
\text { TH-230 } \\
\text { TH-232 } \\
\text { TH-234 } \\
U-234 \\
U-235 \\
U-236 \\
U-238 \\
\text { RA- } 226 \\
\text { RA- } 228\end{array}$ & $\begin{array}{l}Y \\
Y \\
Y \\
y \\
Y \\
Y \\
Y \\
Y \\
W \\
W\end{array}$ & $\begin{array}{l}0.30 \\
0.30 \\
0.30 \\
0.30 \\
0.30 \\
1.00 \\
1.00 \\
0.30 \\
0.30 \\
0.30\end{array}$ & $\begin{array}{l}4.3 \mathrm{E}-07 \\
1.3 \mathrm{E}-06 \\
6.8 \mathrm{E}-08 \\
6.4 \mathrm{E}-05 \\
1.4 \mathrm{E}-05 \\
7.6 \mathrm{E}-07 \\
5.6 \mathrm{E}-07 \\
1.6 \mathrm{E}-05 \\
1.0 \mathrm{E}-08 \\
4.1 \mathrm{E}-08\end{array}$ & $\begin{array}{l}4.3 \mathrm{E}-07 \\
1.3 \mathrm{E}-06 \\
6.8 \mathrm{E}-08 \\
6.4 \mathrm{E}-05 \\
1.4 \mathrm{E}-05 \\
7.6 \mathrm{E}-07 \\
5.6 \mathrm{E}-07 \\
1.6 \mathrm{E}-05 \\
1.0 \mathrm{E}-08 \\
4.1 \mathrm{E}-08\end{array}$ & $\begin{array}{l}8.6 \mathrm{E}-07 \\
2.6 \mathrm{E}-06 \\
1.4 \mathrm{E}-07 \\
1.3 \mathrm{E}-04 \\
2.8 \mathrm{E}-05 \\
1.5 \mathrm{E}-06 \\
1.1 \mathrm{E}-06 \\
3.2 \mathrm{E}-05 \\
2.0 \mathrm{E}-08 \\
8.2 \mathrm{E}-08\end{array}$ \\
\hline
\end{tabular}

\section{SITE INFORMATION}

Temperature: 9 degrees $C$ Precipitation: $\quad 150 \mathrm{~cm} / \mathrm{y}$ Mixing Height: $\quad 950 \mathrm{~m}$ 
Dec 15, 1997 12:38 am

SYNOPSIS

Page 3

SOURCE INFORMATION

$\begin{array}{rrr}\text { Source Number: } & 1 & 2 \\ \text { Stack Height }(\mathrm{m}): & 8.00 & 8.00 \\ \text { Diameter }(\mathrm{m}): & 0.30 & 0.30 \\ \text { P1 ume Rise } & & \\ \text { Momentum (m/s): } & 1.00 \mathrm{E}+00 & 1.00 \mathrm{E}+00 \\ \text { (Exit Velocity) } & & \end{array}$

AGRICULTURAL DATA

Vegetable Milk Meat

Fraction Home Produced: $\quad 0.700 \quad 0.399 \quad 0.442$

$\begin{array}{rlll}\text { Fraction From Assessment Area: } & 0.300 & 0.601 & 0.558 \\ \text { Fraction Imported: } & 0.000 & 0.000 & 0.000\end{array}$

Food Arrays were not generated for this run. Default values used.

DISTANCES USED FOR MAXIMUM INDIVIDUAL ASSESSMENT

$\begin{array}{rrrrrrrrrr}364 & 377 & 569 & 578 & 751 & 880 & 896 & 972 & 1004 & 1111 \\ 1239 & 1243 & 1345 & 1416 & 1553 & & & & & \end{array}$




$$
\begin{gathered}
\text { CA P } 88-P C \\
\text { Version } 1.00 \\
\text { Clean Air Act Assessment Package - } 1988
\end{gathered}
$$

DOSE AND RISK EQUIVALENT SUMMARIES Non-Radon Individual Assessment Dec 15, 1997 12:38 am

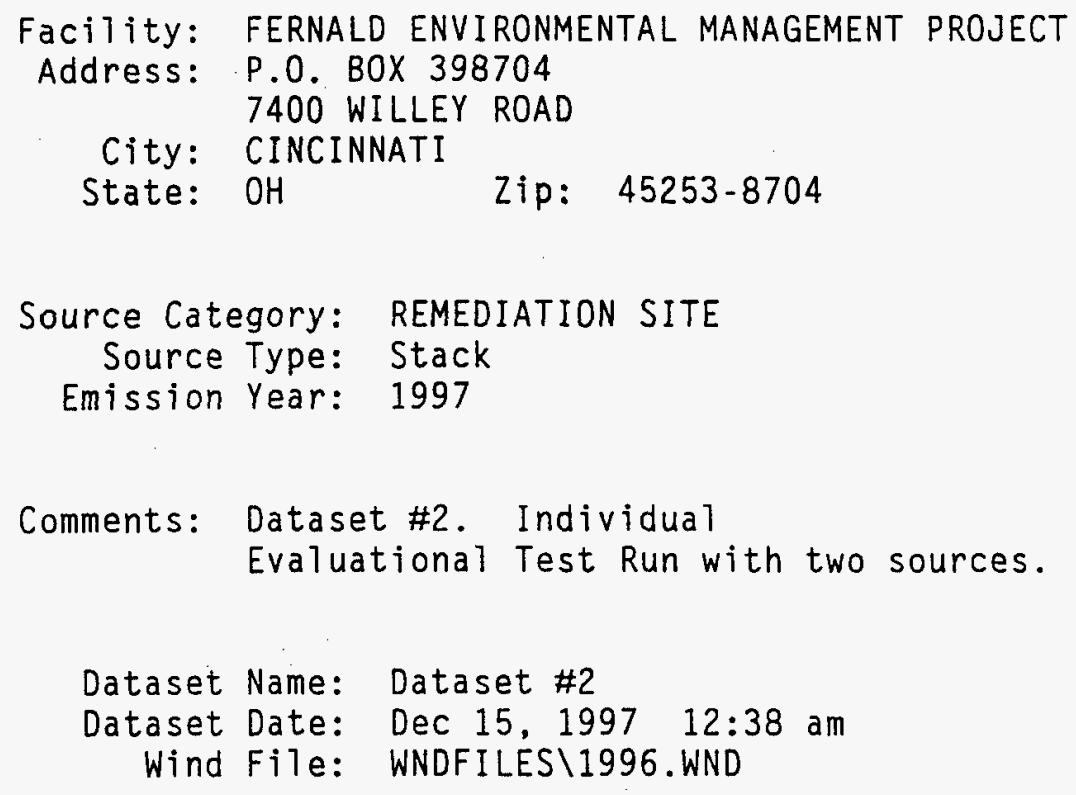


Dec 15, 1997 12:38 am

ORGAN DOSE EQUIVALENT SUMMARY

Organ

Selected

Individua 1

(mrem/y)

$\begin{array}{ll}\text { GONADS } & 1.54 \mathrm{E}-04 \\ \text { BREAST } & 1.74 \mathrm{E}-04 \\ \text { R MAR } & 8.89 \mathrm{E}-03 \\ \text { LUNGS } & 6.22 \mathrm{E}-01 \\ \text { THYROID } & 1.50 \mathrm{E}-04 \\ \text { ENDOST } & 1.15 \mathrm{E}-01 \\ \text { RMNDR } & 3.71 \mathrm{E}-03 \\ & \\ \text { EFFEC } & 8.04 \mathrm{E}-02\end{array}$

PATHWAY EFFECTIVE DOSE EQUIVALENT SUMMARY

Pathway

INGESTION

INHALATION

AIR IMMERSION

GROUND SURFACE

INTERNAL

EXTERNAL

TOTAL
Selected

Individual

(mrem/y)

$2.59 E-03$

$7.78 \mathrm{E}-02$

$3.89 E-09$

2. $92 \mathrm{E}-05$

8.03E-02

2. $92 \mathrm{E}-05$

$8.04 E-02$ 
NUCLIDE EFFECTIVE DOSE EQUIVALENT SUMMARY

Nuctide

TH -228

TH -230

TH- 232

TH -234

U-234

$U-235$

$U-236$

U -238

RA -226

RA- 228

TOTAL
Selected

Individua 1

(mrem/y)

$1.98 E-03$

$5.82 E-03$

$4.43 \mathrm{E}-04$

$6.64 \mathrm{E}-05$

$3.48 \mathrm{E}-02$

$1.06 \mathrm{E}-03$

7. $79 E-04$

$3.54 \mathrm{E}-02$

2.52E-06

3. 73 E- 06

8.04E-02 
CANCER RISK SUMMARY

Cancer

\begin{tabular}{l}
\hline LEUKEMIA \\
BONE \\
THYROID \\
BREAST \\
LUNG \\
STOMACH \\
BOWEL \\
LIVER \\
PANCREAS \\
URINARY \\
OTHER
\end{tabular}

TOTAL

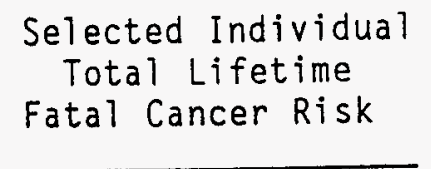

8. 36E- 09

5. $52 \mathrm{E}-09$

3. $40 \mathrm{E}-11$

3. $80 \mathrm{E}-10$

1. $02 E-06$

2. $55 \mathrm{E}-10$

$6.57 E-10$

3. $70 E-10$

1. $50 \mathrm{E}-10$

7. $64 \mathrm{E}-09$

1. $83 \mathrm{E}-10$

$1.04 E-06$

PATHWAY RISK SUMMARY

\begin{tabular}{lc} 
Pathway & $\begin{array}{c}\text { Selected Individual } \\
\text { Total Lifetime } \\
\text { Fatal Cancer Risk }\end{array}$ \\
\cline { 2 - 2 } INGESTION & $1.38 E-08$ \\
INHALATION & $1.02 E-06$ \\
AIR IMMERSION & $8.68 E-14$ \\
GROUND SURFACE & $6.70 \mathrm{E}-10$ \\
INTERNAL & $1.04 \mathrm{E}-06$ \\
EXTERNAL & $6.70 \mathrm{E}-10$ \\
TOTAL & $1.04 \mathrm{E}-06$
\end{tabular}


NUCLIDE RISK SUMMARY

Nuclide

TH -228

$\mathrm{TH}-230$

$\mathrm{TH}-232$

TH -234

$\mathrm{U}-234$

$\mathrm{U}-235$

$\mathrm{U}-236$

$U-238$

$R A-226$

$R A-228$

TOTAL
Selected Individual

Total Lifetime

Fatal Cancer Risk

4.01E-08

$4.88 \mathrm{E}-08$

$2.52 E-09$

2.72E- 09

$4.55 \mathrm{E}-07$

1. $39 E-08$

$1.00 E-08$

$4.66 \mathrm{E}-07$

4. $10 E-11$

4. $41 E-11$

$1.04 E-06$ 
Dec 15, 1997 12:38 am

SUMMARY

Page 5

INDIVIDUAL EFFECTIVE DOSE EQUIVALENT RATE (mrem/y)

(A]l Radionuclides and Pathways)

\begin{tabular}{|c|c|c|c|c|c|c|c|}
\hline \multirow[b]{2}{*}{ Direction } & \multicolumn{7}{|c|}{ Distance $(m)$} \\
\hline & 364 & 377 & 569 & 578 & 751 & 880 & 896 \\
\hline $\begin{array}{r}N \\
N N W \\
N W \\
W N W \\
W \\
W S W \\
S W \\
S S W \\
S \\
S S E \\
S E \\
E S E \\
E \\
E N E \\
N E \\
N N E\end{array}$ & $\begin{array}{l}4.4 \mathrm{E}-02 \\
2.9 \mathrm{E}-02 \\
2.6 \mathrm{E}-02 \\
2.4 \mathrm{E}-02 \\
4.2 \mathrm{E}-02 \\
4.9 \mathrm{E}-02 \\
3.0 \mathrm{E}-02 \\
2.6 \mathrm{E}-02 \\
2.8 \mathrm{E}-02 \\
4.0 \mathrm{E}-02 \\
5.3 \mathrm{E}-02 \\
6.1 \mathrm{E}-02 \\
7.5 \mathrm{E}-02 \\
7.6 \mathrm{E}-02 \\
8.0 \mathrm{E}-02 \\
7.0 \mathrm{E}-02\end{array}$ & $\begin{array}{l}4.2 \mathrm{E}-02 \\
2.8 \mathrm{E}-02 \\
2.5 \mathrm{E}-02 \\
2.3 \mathrm{E}-02 \\
4.0 \mathrm{E}-02 \\
4.7 \mathrm{E}-02 \\
2.8 \mathrm{E}-02 \\
2.5 \mathrm{E}-02 \\
2.7 \mathrm{E}-02 \\
3.8 \mathrm{E}-02 \\
5.1 \mathrm{E}-02 \\
5.9 \mathrm{E}-02 \\
7.2 \mathrm{E}-02 \\
7.3 \mathrm{E}-02 \\
7.7 \mathrm{E}-02 \\
6.7 \mathrm{E}-02\end{array}$ & $\begin{array}{l}2.4 \mathrm{E}-02 \\
1.6 \mathrm{E}-02 \\
1.4 \mathrm{E}-02 \\
1.4 \mathrm{E}-02 \\
2.2 \mathrm{E}-02 \\
2.6 \mathrm{E}-02 \\
1.5 \mathrm{E}-02 \\
1.4 \mathrm{E}-02 \\
1.5 \mathrm{E}-02 \\
2.1 \mathrm{E}-02 \\
2.8 \mathrm{E}-02 \\
3.3 \mathrm{E}-02 \\
4.0 \mathrm{E}-02 \\
4.1 \mathrm{E}-02 \\
4.4 \mathrm{E}-02 \\
3.8 \mathrm{E}-02\end{array}$ & $\begin{array}{l}2.3 \mathrm{E}-02 \\
1.6 \mathrm{E}-02 \\
1.4 \mathrm{E}-02 \\
1.4 \mathrm{E}-02 \\
2.2 \mathrm{E}-02 \\
2.6 \mathrm{E}-02 \\
1.5 \mathrm{E}-02 \\
1.3 \mathrm{E}-02 \\
1.5 \mathrm{E}-02 \\
2.1 \mathrm{E}-02 \\
2.8 \mathrm{E}-02 \\
3.2 \mathrm{E}-02 \\
3.9 \mathrm{E}-02 \\
4.0 \mathrm{E}-02 \\
4.3 \mathrm{E}-02 \\
3.7 \mathrm{E}-02\end{array}$ & $\begin{array}{l}1.5 E-02 \\
1.1 E-02 \\
9.3 E-03 \\
9.2 E-03 \\
1.4 E-02 \\
1.7 E-02 \\
9.9 E-03 \\
8.9 E-03 \\
9.9 E-03 \\
1.4 E-02 \\
1.8 E-02 \\
2.1 E-02 \\
2.6 E-02 \\
2.6 E-02 \\
2.8 E-02 \\
2.5 E-02\end{array}$ & $\begin{array}{l}1.2 \mathrm{E}-02 \\
8.2 \mathrm{E}-03 \\
7.1 \mathrm{E}-03 \\
7.1 \mathrm{E}-03 \\
1.1 \mathrm{E}-02 \\
1.3 \mathrm{E}-02 \\
7.6 \mathrm{E}-03 \\
6.9 \mathrm{E}-03 \\
7.6 \mathrm{E}-03 \\
1.1 \mathrm{E}-02 \\
1.4 \mathrm{E}-02 \\
1.6 \mathrm{E}-02 \\
2.0 \mathrm{E}-02 \\
2.0 \mathrm{E}-02 \\
2.2 \mathrm{E}-02 \\
1.9 \mathrm{E}-02\end{array}$ & $\begin{array}{l}1.1 \mathrm{E}-02 \\
8.0 \mathrm{E}-03 \\
6.9 \mathrm{E}-03 \\
6.9 \mathrm{E}-03 \\
1.1 \mathrm{E}-02 \\
1.3 \mathrm{E}-02 \\
7.3 \mathrm{E}-03 \\
6.7 \mathrm{E}-03 \\
7.4 \mathrm{E}-03 \\
1.0 \mathrm{E}-02 \\
1.4 \mathrm{E}-02 \\
1.6 \mathrm{E}-02 \\
1.9 \mathrm{E}-02 \\
1.9 \mathrm{E}-02 \\
2.1 \mathrm{E}-02 \\
1.8 \mathrm{E}-02\end{array}$ \\
\hline
\end{tabular}

Distance $(m)$

\begin{tabular}{|c|c|c|c|c|c|c|c|}
\hline Direction & 972 & 1004 & 1111 & 1239 & 1243 & 1345 & 1416 \\
\hline $\begin{array}{r}N \\
N N W \\
N W \\
W N W \\
W \\
W S W \\
S W \\
S S W \\
S \\
S S E \\
S E \\
E S E \\
E \\
E N E \\
N E \\
N N E\end{array}$ & $\begin{array}{l}1.0 \mathrm{E}-02 \\
7.0 \mathrm{E}-03 \\
6.1 \mathrm{E}-03 \\
6.0 \mathrm{E}-03 \\
9.2 \mathrm{E}-03 \\
1.1 \mathrm{E}-02 \\
6.4 \mathrm{E}-03 \\
5.8 \mathrm{E}-03 \\
6.4 \mathrm{E}-03 \\
9.0 \mathrm{E}-03 \\
1.2 \mathrm{E}-02 \\
1.4 \mathrm{E}-02 \\
1.7 \mathrm{E}-02 \\
1.7 \mathrm{E}-02 \\
1.8 \mathrm{E}-02 \\
1.6 \mathrm{E}-02\end{array}$ & $\begin{array}{l}9.4 \mathrm{E}-03 \\
6.6 \mathrm{E}-03 \\
5.7 \mathrm{E}-03 \\
5.7 \mathrm{E}-03 \\
8.7 \mathrm{E}-03 \\
1.0 \mathrm{E}-02 \\
6.1 \mathrm{E}-03 \\
5.5 \mathrm{E}-03 \\
6.1 \mathrm{E}-03 \\
8.5 \mathrm{E}-03 \\
1.1 \mathrm{E}-02 \\
1.3 \mathrm{E}-02 \\
1.6 \mathrm{E}-02 \\
1.6 \mathrm{E}-02 \\
1.7 \mathrm{E}-02 \\
1.5 \mathrm{E}-02\end{array}$ & $\begin{array}{l}8.0 \mathrm{E}-03 \\
5.7 \mathrm{E}-03 \\
4.9 \mathrm{E}-03 \\
4.9 \mathrm{E}-03 \\
7.4 \mathrm{E}-03 \\
8.8 \mathrm{E}-03 \\
5.2 \mathrm{E}-03 \\
4.7 \mathrm{E}-03 \\
5.2 \mathrm{E}-03 \\
7.2 \mathrm{E}-03 \\
9.6 \mathrm{E}-03 \\
1.1 \mathrm{E}-02 \\
1.3 \mathrm{E}-02 \\
1.4 \mathrm{E}-02 \\
1.5 \mathrm{E}-02 \\
1.3 \mathrm{E}-02\end{array}$ & $\begin{array}{l}6.8 \mathrm{E}-03 \\
4.8 \mathrm{E}-03 \\
4.1 \mathrm{E}-03 \\
4.1 \mathrm{E}-03 \\
6.2 \mathrm{E}-03 \\
7.4 \mathrm{E}-03 \\
4.4 \mathrm{E}-03 \\
4.0 \mathrm{E}-03 \\
4.4 \mathrm{E}-03 \\
6.1 \mathrm{E}-03 \\
8.0 \mathrm{E}-03 \\
9.4 \mathrm{E}-03 \\
1.1 \mathrm{E}-02 \\
1.1 \mathrm{E}-02 \\
1.2 \mathrm{E}-02 \\
1.1 \mathrm{E}-02\end{array}$ & $\begin{array}{l}6.7 E-03 \\
4.7 E-03 \\
4.1 E-03 \\
4.1 E-03 \\
6.2 E-03 \\
7.4 E-03 \\
4.3 E-03 \\
4.0 E-03 \\
4.4 E-03 \\
6.1 E-03 \\
8.0 E-03 \\
9.4 E-03 \\
1.1 E-02 \\
1.1 E-02 \\
1.2 E-02 \\
1.1 E-02\end{array}$ & $\begin{array}{l}5.9 \mathrm{E}-03 \\
4.2 \mathrm{E}-03 \\
3.6 \mathrm{E}-03 \\
3.6 \mathrm{E}-03 \\
5.5 \mathrm{E}-03 \\
6.5 \mathrm{E}-03 \\
3.8 \mathrm{E}-03 \\
3.5 \mathrm{E}-03 \\
3.9 \mathrm{E}-03 \\
5.3 \mathrm{E}-03 \\
7.0 \mathrm{E}-03 \\
8.3 \mathrm{E}-03 \\
9.9 \mathrm{E}-03 \\
1.0 \mathrm{E}-02 \\
1.1 \mathrm{E}-02 \\
9.4 \mathrm{E}-03\end{array}$ & $\begin{array}{l}5.5 E-03 \\
3.9 E-03 \\
3.4 E-03 \\
3.4 E-03 \\
5.0 E-03 \\
6.0 E-03 \\
3.5 E-03 \\
3.2 E-03 \\
3.6 E-03 \\
4.9 E-03 \\
6.5 E-03 \\
7.6 E-03 \\
9.1 E-03 \\
9.2 E-03 \\
9.9 E-03 \\
8.7 E-03\end{array}$ \\
\hline
\end{tabular}


Dec $15,1997 \quad 12: 38$ am

SUMMARY

Page 6

INDIVIDUAL EFFECTIVE DOSE EQUIVALENT RATE (mrem/y)

(AII Radionuclides and Pathways)

\begin{tabular}{rl} 
& \\
& \\
\cline { 2 - 2 } Direction & 1553 \\
\hline$N$ & $4.7 \mathrm{E}-03$ \\
NNW & $3.3 \mathrm{E}-03$ \\
$N W$ & $2.9 \mathrm{E}-03$ \\
WNW & $2.9 \mathrm{E}-03$ \\
$W$ & $4.3 \mathrm{E}-03$ \\
WSW & $5.2 \mathrm{E}-03$ \\
SW & $3.1 \mathrm{E}-03$ \\
SSW & $2.8 \mathrm{E}-03$ \\
$\mathrm{~S}$ & $3.1 \mathrm{E}-03$ \\
SSE & $4.3 \mathrm{E}-03$ \\
$\mathrm{SE}$ & $5.6 \mathrm{E}-03$ \\
$\mathrm{ESE}$ & $6.6 \mathrm{E}-03$ \\
$\mathrm{E}$ & $7.8 \mathrm{E}-03$ \\
$\mathrm{ENE}$ & $7.9 \mathrm{E}-03$ \\
$N E$ & $8.5 \mathrm{E}-03$ \\
$\mathrm{NNE}$ & $7.5 \mathrm{E}-03$ \\
\hline
\end{tabular}


INDIVIDUAL LIFETIME RISK (deaths)

(All Radionuclides and Pathways)

\begin{tabular}{|c|c|c|c|c|c|c|c|}
\hline \multirow[b]{2}{*}{ Direction } & \multicolumn{6}{|c|}{ Distance $(m)$} & \multirow[b]{2}{*}{896} \\
\hline & 364 & 377 & 569 & 578 & 751 & 880 & \\
\hline $\begin{array}{r}N \\
N N W \\
N W \\
W N W \\
W \\
W S W \\
S W \\
S S W \\
S \\
S S E \\
S E \\
E S E \\
E \\
E N E \\
N E \\
N N E\end{array}$ & $\begin{array}{l}5.7 \mathrm{E}-07 \\
3.8 \mathrm{E}-07 \\
3.3 \mathrm{E}-07 \\
3.1 \mathrm{E}-07 \\
5.4 \mathrm{E}-07 \\
6.4 \mathrm{E}-07 \\
3.8 \mathrm{E}-07 \\
3.3 \mathrm{E}-07 \\
3.6 \mathrm{E}-07 \\
5.2 \mathrm{E}-07 \\
6.9 \mathrm{E}-07 \\
7.9 \mathrm{E}-07 \\
9.7 \mathrm{E}-07 \\
9.9 \mathrm{E}-07 \\
1.0 \mathrm{E}-06 \\
9.0 \mathrm{E}-07\end{array}$ & $\begin{array}{l}5.5 E-07 \\
3.6 \mathrm{E}-07 \\
3.2 \mathrm{E}-07 \\
3.0 \mathrm{E}-07 \\
5.1 \mathrm{E}-07 \\
6.1 \mathrm{E}-07 \\
3.7 \mathrm{E}-07 \\
3.2 \mathrm{E}-07 \\
3.5 \mathrm{E}-07 \\
5.0 \mathrm{E}-07 \\
6.6 \mathrm{E}-07 \\
7.6 \mathrm{E}-07 \\
9.3 \mathrm{E}-07 \\
9.4 \mathrm{E}-07 \\
9.9 \mathrm{E}-07 \\
8.6 \mathrm{E}-07\end{array}$ & $\begin{array}{l}3.1 \mathrm{E}-07 \\
2.1 \mathrm{E}-07 \\
1.8 \mathrm{E}-07 \\
1.8 \mathrm{E}-07 \\
2.9 \mathrm{E}-07 \\
3.4 \mathrm{E}-07 \\
2.0 \mathrm{E}-07 \\
1.8 \mathrm{E}-07 \\
2.0 \mathrm{E}-07 \\
2.8 \mathrm{E}-07 \\
3.7 \mathrm{E}-07 \\
4.3 \mathrm{E}-07 \\
5.2 \mathrm{E}-07 \\
5.3 \mathrm{E}-07 \\
5.6 \mathrm{E}-07 \\
4.9 \mathrm{E}-07\end{array}$ & $\begin{array}{l}3.0 \mathrm{E}-07 \\
2.0 \mathrm{E}-07 \\
1.8 \mathrm{E}-07 \\
1.7 \mathrm{E}-07 \\
2.8 \mathrm{E}-07 \\
3.3 \mathrm{E}-07 \\
1.9 \mathrm{E}-07 \\
1.7 \mathrm{E}-07 \\
1.9 \mathrm{E}-07 \\
2.7 \mathrm{E}-07 \\
3.6 \mathrm{E}-07 \\
4.2 \mathrm{E}-07 \\
5.1 \mathrm{E}-07 \\
5.1 \mathrm{E}-07 \\
5.5 \mathrm{E}-07 \\
4.8 \mathrm{E}-07\end{array}$ & $\begin{array}{l}2.0 \mathrm{E}-07 \\
1.4 \mathrm{E}-07 \\
1.2 \mathrm{E}-07 \\
1.2 \mathrm{E}-07 \\
1.8 \mathrm{E}-07 \\
2.2 \mathrm{E}-07 \\
1.3 \mathrm{E}-07 \\
1.1 \mathrm{E}-07 \\
1.3 \mathrm{E}-07 \\
1.8 \mathrm{E}-07 \\
2.4 \mathrm{E}-07 \\
2.8 \mathrm{E}-07 \\
3.3 \mathrm{E}-07 \\
3.4 \mathrm{E}-07 \\
3.6 \mathrm{E}-07 \\
3.2 \mathrm{E}-07\end{array}$ & $\begin{array}{l}1.5 \mathrm{E}-07 \\
1.0 \mathrm{E}-07 \\
9.1 \mathrm{E}-08 \\
9.0 \mathrm{E}-08 \\
1.4 \mathrm{E}-07 \\
1.7 \mathrm{E}-07 \\
9.6 \mathrm{E}-08 \\
8.7 \mathrm{E}-08 \\
9.7 \mathrm{E}-08 \\
1.4 \mathrm{E}-07 \\
1.8 \mathrm{E}-07 \\
2.1 \mathrm{E}-07 \\
2.5 \mathrm{E}-07 \\
2.6 \mathrm{E}-07 \\
2.8 \mathrm{E}-07 \\
2.4 \mathrm{E}-07\end{array}$ & $\begin{array}{l}1.5 E-07 \\
1.0 E-07 \\
8.8 E-08 \\
8.8 E-08 \\
1.3 E-07 \\
1.6 E-07 \\
9.3 E-08 \\
8.4 E-08 \\
9.4 E-08 \\
1.3 E-07 \\
1.7 E-07 \\
2.1 E-07 \\
2.5 E-07 \\
2.5 E-07 \\
2.7 E-07 \\
2.3 E-07\end{array}$ \\
\hline \multicolumn{8}{|c|}{ Distance (m) } \\
\hline Direction & 972 & 1004 & 1111 & 1239 & 1243 & 1345 & 1416 \\
\hline $\begin{array}{r}N \\
N N W \\
N W \\
W N W \\
W \\
W S W \\
S W \\
S S W \\
S \\
S S E \\
S E \\
E S E \\
E \\
E N E \\
N E \\
N N E\end{array}$ & $\begin{array}{l}1.3 \mathrm{E}-07 \\
8.8 \mathrm{E}-08 \\
7.6 \mathrm{E}-08 \\
7.6 \mathrm{E}-08 \\
1.2 \mathrm{E}-07 \\
1.4 \mathrm{E}-07 \\
8.1 \mathrm{E}-08 \\
7.3 \mathrm{E}-08 \\
8.1 \mathrm{E}-08 \\
1.1 \mathrm{E}-07 \\
1.5 \mathrm{E}-07 \\
1.8 \mathrm{E}-07 \\
2.1 \mathrm{E}-07 \\
2.2 \mathrm{E}-07 \\
2.3 \mathrm{E}-07 \\
2.0 \mathrm{E}-07\end{array}$ & $\begin{array}{l}1.2 \mathrm{E}-07 \\
8.4 \mathrm{E}-08 \\
7.2 \mathrm{E}-08 \\
7.2 \mathrm{E}-08 \\
1.1 \mathrm{E}-07 \\
1.3 \mathrm{E}-07 \\
7.6 \mathrm{E}-08 \\
6.9 \mathrm{E}-08 \\
7.7 \mathrm{E}-08 \\
1.1 \mathrm{E}-07 \\
1.4 \mathrm{E}-07 \\
1.7 \mathrm{E}-07 \\
2.0 \mathrm{E}-07 \\
2.1 \mathrm{E}-07 \\
2.2 \mathrm{E}-07 \\
1.9 \mathrm{E}-07\end{array}$ & $\begin{array}{l}1.0 \mathrm{E}-07 \\
7.1 \mathrm{E}-08 \\
6.2 \mathrm{E}-08 \\
6.1 \mathrm{E}-08 \\
9.4 \mathrm{E}-08 \\
1.1 \mathrm{E}-07 \\
6.5 \mathrm{E}-08 \\
5.9 \mathrm{E}-08 \\
6.6 \mathrm{E}-08 \\
9.2 \mathrm{E}-08 \\
1.2 \mathrm{E}-07 \\
1.4 \mathrm{E}-07 \\
1.7 \mathrm{E}-07 \\
1.7 \mathrm{E}-07 \\
1.9 \mathrm{E}-07 \\
1.6 \mathrm{E}-07\end{array}$ & $\begin{array}{l}8.6 \mathrm{E}-08 \\
6.0 \mathrm{E}-08 \\
5.2 \mathrm{E}-08 \\
5.2 \mathrm{E}-08 \\
7.9 \mathrm{E}-08 \\
9.4 \mathrm{E}-08 \\
5.4 \mathrm{E}-08 \\
5.0 \mathrm{E}-08 \\
5.5 \mathrm{E}-08 \\
7.7 \mathrm{E}-08 \\
1.0 \mathrm{E}-07 \\
1.2 \mathrm{E}-07 \\
1.4 \mathrm{E}-07 \\
1.5 \mathrm{E}-07 \\
1.6 \mathrm{E}-07 \\
1.4 \mathrm{E}-07\end{array}$ & $\begin{array}{l}8.5 \mathrm{E}-08 \\
5.9 \mathrm{E}-08 \\
5.1 \mathrm{E}-08 \\
5.1 \mathrm{E}-08 \\
7.8 \mathrm{E}-08 \\
9.4 \mathrm{E}-08 \\
5.4 \mathrm{E}-08 \\
4.9 \mathrm{E}-08 \\
5.5 \mathrm{E}-08 \\
7.7 \mathrm{E}-08 \\
1.0 \mathrm{E}-07 \\
1.2 \mathrm{E}-07 \\
1.4 \mathrm{E}-07 \\
1.5 \mathrm{E}-07 \\
1.6 \mathrm{E}-07 \\
1.4 \mathrm{E}-07\end{array}$ & $\begin{array}{l}7.5 \mathrm{E}-08 \\
5.2 \mathrm{E}-08 \\
4.5 \mathrm{E}-08 \\
4.5 \mathrm{E}-08 \\
6.9 \mathrm{E}-08 \\
8.2 \mathrm{E}-08 \\
4.8 \mathrm{E}-08 \\
4.3 \mathrm{E}-08 \\
4.8 \mathrm{E}-08 \\
6.7 \mathrm{E}-08 \\
8.9 \mathrm{E}-08 \\
1.1 \mathrm{E}-07 \\
1.3 \mathrm{E}-07 \\
1.3 \mathrm{E}-07 \\
1.4 \mathrm{E}-07 \\
1.2 \mathrm{E}-07\end{array}$ & $\begin{array}{l}5.9 E-08 \\
4.8 E-08 \\
4.1 E-08 \\
4.2 E-08 \\
6.3 E-08 \\
7.6 E-08 \\
4.4 E-08 \\
4.0 E-08 \\
4.4 E-08 \\
6.2 E-08 \\
8.2 E-08 \\
9.7 E-08 \\
1.2 E-07 \\
1.2 E-07 \\
1.3 E-07 \\
1.1 E-07\end{array}$ \\
\hline
\end{tabular}


INDIVIDUAL LIFETIME RISK (deaths)

(A1l Radionuclides and Pathways)

\begin{tabular}{|c|c|}
\hline & Distance (m) \\
\hline Direction & 1553 \\
\hline $\begin{array}{r}N \\
N N W \\
N W \\
W N W \\
W \\
W S W \\
S W \\
S S W \\
S \\
S S E \\
S E \\
E S E \\
E \\
E N E \\
N E \\
N N E\end{array}$ & $\begin{array}{l}5.9 E-08 \\
4.1 E-08 \\
3.6 E-08 \\
3.6 E-08 \\
5.4 E-08 \\
6.5 E-08 \\
3.8 E-08 \\
3.4 E-08 \\
3.8 E-08 \\
5.3 E-08 \\
7.0 E-08 \\
8.3 E-08 \\
9.9 E-08 \\
1.0 E-07 \\
1.1 E-07 \\
9.5 E-08\end{array}$ \\
\hline
\end{tabular}




\section{A P 88 - P C \\ Version 2.00}

Clean Air Act Assessment Package - 1988

$$
\begin{gathered}
\text { S Y N O P S I S R E P O R T } \\
\text { Non-Radon Individual Assessment } \\
\text { Dec 16, } 199702: 10 \mathrm{pm}
\end{gathered}
$$

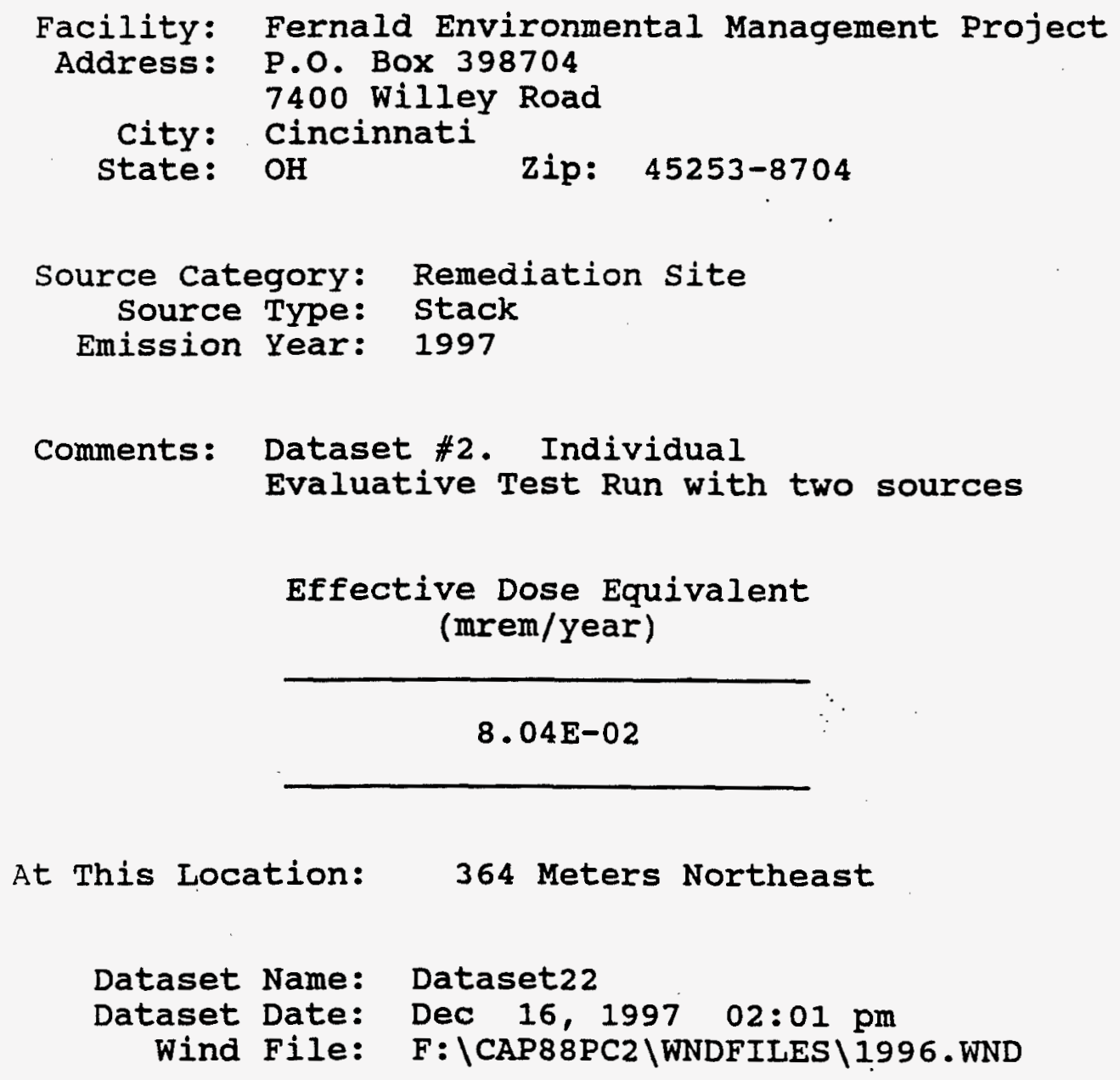




\section{MAXIMALLY EXPOSED INDIVIDUAL}

Location of The Individual: Lifetime Fatal Cancer Risk:

364 Meters Northeast

$$
1.04 \mathrm{E}-06
$$

ORGAN DOSE EQUIVALENT SUMMARY

\begin{tabular}{lc} 
Organ & $\begin{array}{c}c \\
\text { Dose } \\
\text { Equivalent } \\
(\text { mrem } / y)\end{array}$ \\
\cline { 2 - 2 } GONADS & $1.54 \mathrm{E}-04$ \\
BREAST & $1.74 \mathrm{E}-04$ \\
R MAR & $8.89 \mathrm{E}-03$ \\
LUNGS & $6.22 \mathrm{E}-01$ \\
THYROID & $1.50 \mathrm{E}-04$ \\
ENDOST & $1.15 \mathrm{E}-01$ \\
RMNDR & $3.71 \mathrm{E}-03$ \\
EFFEC & $8.04 \mathrm{E}-02$
\end{tabular}




\section{RADIONUCLIDE EMISSIONS DURING THE YEAR 1997}

Source source

\#1 \#2 TOTAL

\begin{tabular}{|c|c|c|c|c|c|}
\hline Nuclide & Class & Size & $\mathrm{Ci} / \mathrm{Y}$ & $\mathrm{Ci} / \mathrm{Y}$ & $\mathrm{Ci} / \mathrm{Y}$ \\
\hline$T H-228$ & $\mathrm{Y}$ & 0.30 & 4. $3 E-07$ & $4.3 E-07$ & $8.6 \mathrm{E}-07$ \\
\hline $7 \mathrm{H}-2: 0$ & $Y$ & 0.30 & $1.3 E-06$ & $1.3 E-06$ & $2.6 \mathrm{E}-06$ \\
\hline $\mathrm{TH}-2: 2$ & $Y$ & 0.30 & $6.8 E-08$ & $6.8 E-08$ & $1.4 \mathrm{E}-07$ \\
\hline $\mathrm{T} H-2: \hat{4}$ & $\bar{Y}$ & 0.30 & $6.4 \mathrm{E}-05$ & $6.4 \mathrm{E}-05$ & 1. $3 E-04$ \\
\hline$(i-23 ;$ & $Y$ & 0.30 & $1.4 \mathrm{E}-05$ & $1.4 \mathrm{E}-05$ & $2.8 E-05$ \\
\hline$U-235$ & $\mathrm{Y}$ & 1.00 & $7.6 E-07$ & $7.6 E-07$ & $1.5 \mathrm{E}-06$ \\
\hline$U-236$ & $Y$ & 1.00 & $5.6 E-07$ & $5.6 E-07$ & 1. $1 \mathrm{E}-06$ \\
\hline$U-238$ & $Y$ & 0.30 & 1. $6 E-05$ & $1.6 \mathrm{E}-05$ & $3.2 E-05$ \\
\hline$R A-226$ & $W$ & 0.30 & $1.0 \mathrm{E}-08$ & $1.0 \mathrm{E}-08$ & 2. $0 E-08$ \\
\hline$R A-228$ & W & 0.30 & $4.1 E-08$ & $4.1 E-08$ & $8.2 E-08$ \\
\hline
\end{tabular}

\section{SITE INFORMATION}

Temperature: Precipitation: Mixing Height:
9 degrees $\mathrm{C}$ $150 \mathrm{~cm} / \mathrm{Y}$ $950 \mathrm{~m}$ 
ilec $11,199702: 10 \mathrm{pm}$

SOURCE INFORMATION

\begin{tabular}{|c|c|c|}
\hline Source Number: & & \\
\hline $\begin{array}{r}\text { stack Height }(m): \\
\text { Diameter }(m):\end{array}$ & $\begin{array}{l}8 . \\
0 .\end{array}$ & $\begin{array}{l}8 . \\
0 .\end{array}$ \\
\hline $\begin{array}{l}\text { Plume Rise } \\
\text { Momentum (m/s): } \\
\text { (Exit Velocity) }\end{array}$ & 1. & 1. \\
\hline
\end{tabular}

AGRICULTURAL DATA

\begin{tabular}{|c|c|c|c|}
\hline & Vegetable & Milk & Meat \\
\hline $\begin{array}{l}\text { Fraction Home Produced: } \\
\text { Fraction From Assessment Area: } \\
\text { Fraction Imported: }\end{array}$ & $\begin{array}{l}0.700 \\
0.300 \\
0.000\end{array}$ & $\begin{array}{l}0.399 \\
0.601 \\
0.000\end{array}$ & $\begin{array}{l}0.442 \\
0.558 \\
0.000\end{array}$ \\
\hline
\end{tabular}

Food Arrays were not generated for this run. Default values used.

DISTANCES (M) USED FOR MAXIMUM INDIVIDUAL ASSESSMENT

$\begin{array}{rrrrrrrrrr}364 & 377 & 569 & 578 & 751 & 880 & 896 & 972 & 1004 & 1111 . \\ 1239 & 1243 & 1345 & 1416 & 1553 & & & & & \end{array}$


C A P 8 8 - P C

Version 2.00

Clean Air Act Assessment Package - 1988

D O S E A N D R I S K E Q U I V A I E N T S U M M A R I E S

Non-Radon Individual Assessment

Dec 16, $199702: 10 \mathrm{pm}$

$\begin{aligned} & \text { Facility: Fernald Environmental Management Project } \\ & \text { Address: } \text { P.O. Box } 398704 \\ & 7400 \text { Willey Road } \\ & \text { City: Cincinnati } \text { Zip: } 45253-8704 \\ & \text { State: } \text { OH }\end{aligned}$

Source Category: Remediation site

Source Type: stack

Emission Year: 1997

Comments: Dataset \#2. Individual

Evaluative Test Run with two sources

Dataset Name:

Dataset22

Dataset Date: Dec 16, 1997 02:01 pm

Wind File: F:\CAP88PC2 \WNDFILES\1996.WND 


\begin{tabular}{lr} 
ORGAN DOSE EQUIVALENT SUMMARY \\
Organ & $\begin{array}{c}\text { Selected } \\
\text { Individual } \\
(\text { mrem/y) }\end{array}$ \\
\cline { 2 - 2 } GONADS & $1.54 \mathrm{E}-04$ \\
BREAST & $1.74 \mathrm{E}-04$ \\
R MAR & $8.89 \mathrm{E}-03$ \\
LUNGS & $6.22 \mathrm{E}-01$ \\
THYROID & $1.50 \mathrm{E}-04$ \\
ENDOST & $1.15 \mathrm{E}-01$ \\
RMNDR & $3.71 \mathrm{E}-03$ \\
EFFEC & $8.04 \mathrm{E}-02$
\end{tabular}

PATHWAY EFFECTIVE DOSE EQUIVALENT SUMMARY

Selected

Individual

Pathway

(mrem/y)

INGESTION

2.59E-03

INHAIATION

$7.78 \mathrm{E}-02$

AIR IMMERSION

$3.89 E-09$

GROUND SURFACE

$2.92 E-05$

INTERNAL

EXTERNAL

8. 03E-02

2. $92 E-05$

TOTAL

8. $04 E-02$ 
vec $16,199702: 10 \mathrm{pm}$

NUCLIDE EFFECTIVE DOSE EQUIVALENT SUMMARY

Nuclide

$\mathrm{TH}-228$

TH -230

$\mathrm{TH}-232$

TH -234

$\mathrm{U}-234$

$\mathrm{U}-235$

$\mathrm{U}-236$

$\mathrm{U}-238$

$R A-226$

$R A-228$

TOTAL
Selected

Individual

(mrem/y)

1. $98 \mathrm{E}-03$

$5.82 \mathrm{E}-03$

4. $43 \mathrm{E}-04$

$6.64 \mathrm{E}-05$

3. $48 \mathrm{E}-02$

1. $06 \mathrm{E}-03$

7. 79E-04

3. $54 \mathrm{E}-02$

2. 52E-06

3. $73 \mathrm{E}-06$

$8.04 E-02$ 


\begin{tabular}{lc} 
& $\begin{array}{c}\text { Selected Individual } \\
\text { Total Lifetime } \\
\text { Fatal Cancer Risk }\end{array}$ \\
\hline LEUKEMIA & $8.36 \mathrm{E}-09$ \\
BONE & $5.52 \mathrm{E}-09$ \\
THYROID & $3.40 \mathrm{E}-11$ \\
BREAST & $3.80 \mathrm{E}-10$ \\
LUNG & $1.02 \mathrm{E}-06$ \\
STOMACH & $2.55 \mathrm{E}-10$ \\
BOWEL & $6.57 \mathrm{E}-10$ \\
LIVER & $3.70 \mathrm{E}-10$ \\
PANCREAS & $1.50 \mathrm{E}-10$ \\
URINARY & $7.64 \mathrm{E}-09$ \\
OTHER & $1.83 \mathrm{E}-10$ \\
TOTAL & $1.04 \mathrm{E}-06$
\end{tabular}

PATHWAY RISK SUMMARY

Pathway

INGESTION

INHALATION

AIR IMMERSION

GROUND SURFACE

INTERNAL

EXTERNAL

TOTAL
Selected Individual Total Lifetime Fatal Cancer Risk

1. $.38 \mathrm{E}-08$

$1.02 \mathrm{E}-06$

8. $68 \mathrm{E}-14$

$6.70 \mathrm{E}-10$

1. $04 \mathrm{E}-06$

$6.70 \mathrm{E}-10$

$1.04 \mathrm{E}-06$ 


\section{NUCLIDE RISK SUMMARY}

Selected Individual

Total Iifetime

Nuclide

TH-228

$\mathrm{TH}-230$

$\mathrm{TH}-232$

$\mathrm{TH}-234$

$\mathrm{U}-234$

$\mathrm{U}-235$

$\mathrm{U}-236$

$\mathrm{U}-238$

$R A-226$

$R A-228$

TOTAL
Fatal Cancer Risk

$4.01 E-08$

$4.88 \mathrm{E}-08$

2. $52 E-09$

2.72E-09

4. 55E-07

1. $39 \mathrm{E}-08$

$1.00 \mathrm{E}-08$

4. 66E-07

4. $10 \mathrm{E}-11$

4. $41 \mathrm{E}-11$

1. $04 \mathrm{E}-06$ 
INDIVIDUAL EFFECTIVE DOSE EQUIVALENT RATE (mrem/Y) (All Radionuclides and Pathways)

\section{Distance (m)}

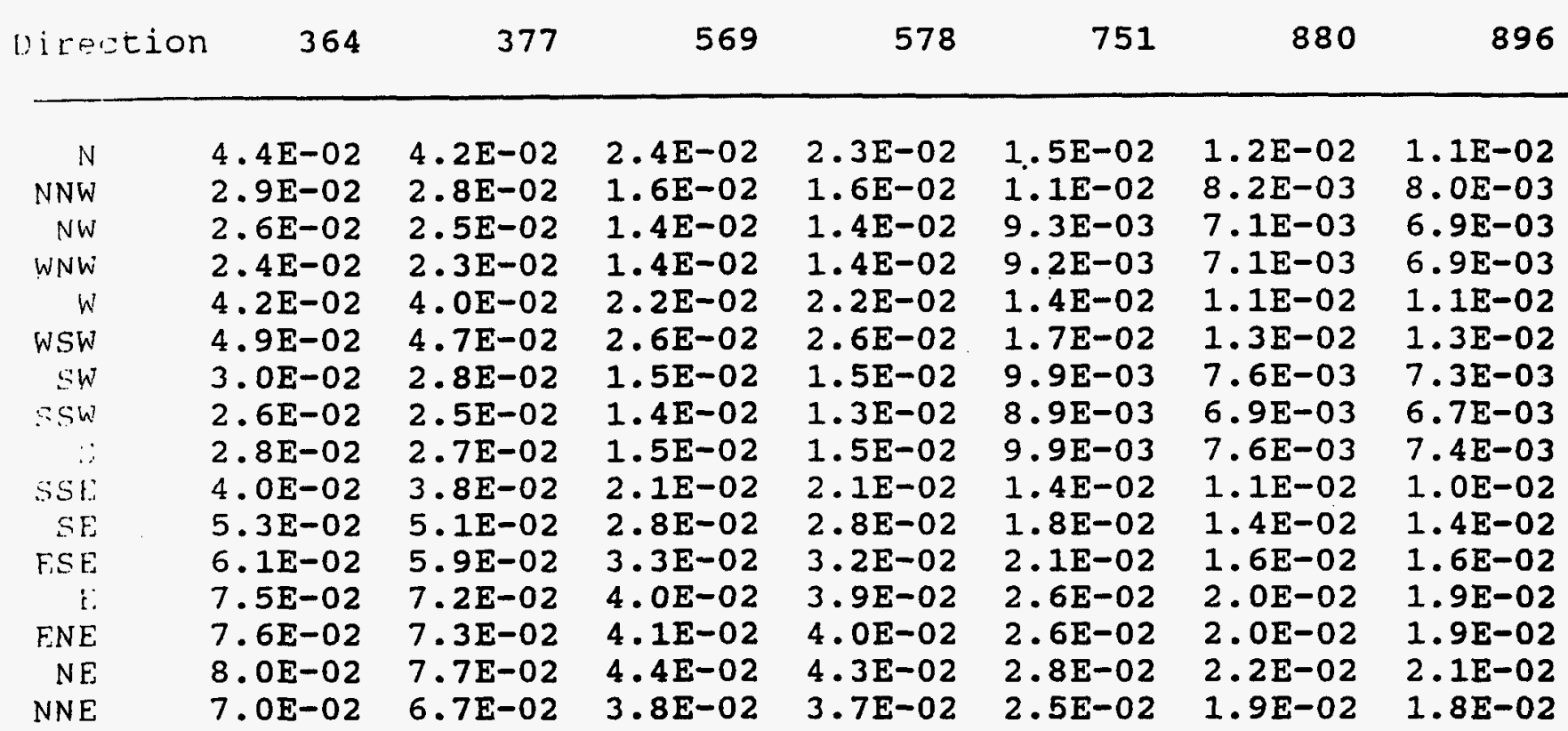

Distance (m)

$\begin{array}{llllllll}\text { Direction } & 972 & 1004 & 1111 & 1239 & 1243 & 1345 & 1416\end{array}$

\begin{tabular}{|c|c|c|c|c|c|c|c|}
\hline$N$ & 1. $0 \mathrm{E}-02$ & $9.4 \mathrm{E}-03$ & $8.0 E-03$ & $6.8 E-03$ & $6: 7 E-03$ & $5.9 E-03$ & $5.5 E-03$ \\
\hline NNW & $7.0 E-03$ & $6.6 \mathrm{E}-03$ & $5.7 E-03$ & $4.8 \mathrm{E}-03$ & $4.7 E-03$ & $4.2 E-03$ & $3.9 E-03$ \\
\hline $\mathrm{NW}$ & $6.1 E-03$ & $5.7 E-03$ & $4.9 E-03$ & $4 \cdot 1 \cdot E-03$ & $4.1 E-03$ & $3.6 E-03$ & $3.4 \mathrm{E}-03$ \\
\hline WNW & $6.0 E-03$ & $5.7 E-03$ & $4.9 \mathrm{E}-03$ & $4 \cdot 1 \mathrm{E}-03$ & $4.1 E-03$ & $3.6 \mathrm{E}-03$ & $3.4 \mathrm{E}-03$ \\
\hline$\Leftrightarrow$ & $9.2 \mathrm{E}-03$ & $8.7 E-03$ & $7.4 \mathrm{E}-03$ & $6.2 \mathrm{E}-03$ & $6.2 \mathrm{E}-03$ & $5.5 E-03$ & $5.0 E-03$ \\
\hline WSW & 1. $1 E-02$ & 1. $0 \mathrm{E}-02$ & $8.8 E-03$ & $7.4 E-03$ & $7.4 E-03$ & $6.5 E-03$ & $6.0 E-03$ \\
\hline$S h$ & $6.4 E-03$ & $6.1 E-03$ & $5.2 E-03$ & $4.4 E-03$ & $4.3 E-03$ & $3.8 E-03$ & $3.5 E-03$ \\
\hline$s s h^{\prime}$ & $5.8 E-03$ & $5.5 E-03$ & $4.7 \mathrm{E}-03$ & 4. $0 \mathrm{E}-03$ & $4.0 E-03$ & $3.5 E-03$ & $3.2 E-03$ \\
\hline 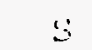 & $6.4 E-03$ & $6.1 \mathrm{E}-03$ & $5.2 E-03$ & $4.4 \mathrm{E}-03$ & $4: 4 \mathrm{E}-03$ & $3.9 E-03$ & $3.6 \mathrm{E}-03$ \\
\hline$\because S E$ & $9.0 \mathrm{E}-03$ & $8.5 E-03$ & $7.2 E-03$ & $6.1 E-03$ & $6.1 E-03$ & $5.3 E-03$ & $4.9 E-03$ \\
\hline$S r$ & $1.2 \mathrm{E}-02$ & 1. $1 \mathrm{E}-02$ & $9.6 \mathrm{E}-03$ & $8.0 E-03$ & $8.0 E-03$ & $7.0 \mathrm{E}-03$ & $6.5 \mathrm{E}-03$ \\
\hline F.SE & $1.4 \mathrm{E}-02$ & 1. $3 E-02$ & $1.1 \mathrm{E}-02$ & $9.4 \mathrm{E}-03$ & $9.4 \mathrm{E}-03$ & $8.3 E-03$ & $7.6 \mathrm{E}-03$ \\
\hline$E$ & $1.7 \mathrm{E}-02$ & $1.6 \mathrm{E}-02$ & 1. $3 E-02$ & 1. IE-02 & 1. $1 E-02$ & $9.9 E-03$ & $9.1 E-03$ \\
\hline ENE & $1.7 \mathrm{E}-02$ & 1. $6 \mathrm{E}-02$ & $1.4 \mathrm{E}-02$ & 1. $1 E-02$ & 1. $1 \mathrm{E}-02$ & 1. $0 \mathrm{E}-02$ & $9.2 \mathrm{E}-03$ \\
\hline $\mathrm{NE}$ & $1.8 \mathrm{E}-02$ & $1.7 \mathrm{E}-02$ & $1.5 \mathrm{E}-02$ & 1. $2 \mathrm{E}-02$ & $1.2 E-02$ & 1. $1 \mathrm{E}-02$ & $9.9 E-03$ \\
\hline NNE & $1.6 \mathrm{E}-02$ & $1.5 E-02$ & $1.3 E-02$ & $1.1 \mathrm{E}-02$ & 1. $1 E-02$ & $9.4 \mathrm{E}-03$ & $8.7 E-03$ \\
\hline
\end{tabular}


INDIVIDUAL EFFECTIVE DOSE EQUIVALENT RATE (mrem/Y)

(All Radionuclides and Pathways)

\section{Distance (m)}

\section{Direction 1553}

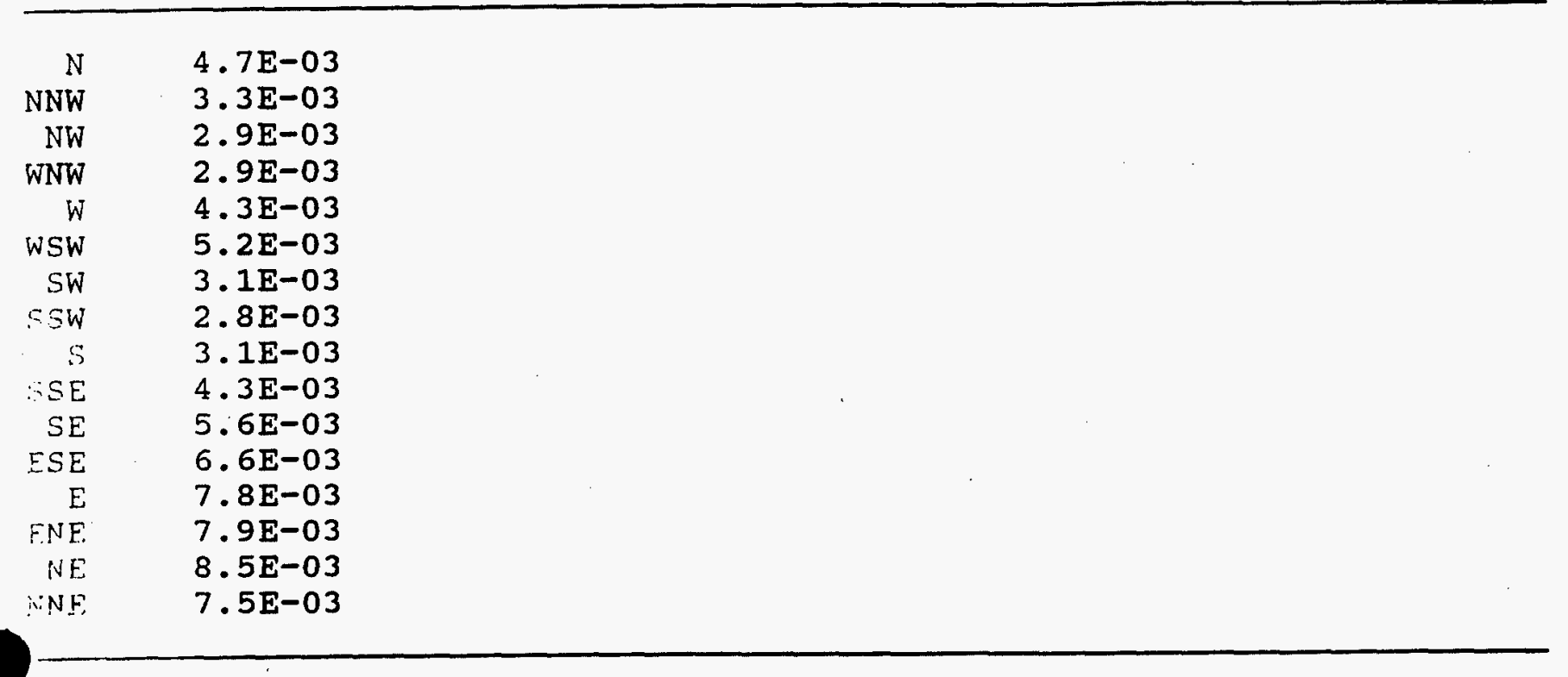


Dec $16,199702: 10 \mathrm{pm}$

INDIVIDUAL LIFETIME RISK (deaths)

(AIl Radionuclides and Pathways)

Distance $(\mathrm{m})$

\begin{tabular}{|c|c|c|c|c|c|c|c|}
\hline nirortion & 364 & 377 & 569 & 578 & 751 & 880 & 896 \\
\hline $\begin{array}{r}: 1 \\
N N W \\
N W \\
W N W \\
\vdots \\
W S W \\
S W \\
S S W \\
\vdots \\
S S E \\
S E \\
P S E \\
E \\
\text { ENE } \\
N E \\
N N E\end{array}$ & $\begin{array}{l}5.7 \mathrm{E}-07 \\
3.8 \mathrm{E}-07 \\
3.3 \mathrm{E}-07 \\
3.1 \mathrm{E}-07 \\
5.4 \mathrm{E}-07 \\
6.4 \mathrm{E}-07 \\
3.8 \mathrm{E}-07 \\
3.3 \mathrm{E}-07 \\
3.6 \mathrm{E}-07 \\
5.2 \mathrm{E}-07 \\
6.9 \mathrm{E}-07 \\
7.9 \mathrm{E}-07 \\
9.7 \mathrm{E}-07 \\
9.9 \mathrm{E}-07 \\
1.0 \mathrm{E}-06 \\
9.0 \mathrm{E}-07\end{array}$ & $\begin{array}{l}5.5 \mathrm{E}-07 \\
3.6 \mathrm{E}-07 \\
3.2 \mathrm{E}-07 \\
3.0 \mathrm{E}-07 \\
5.1 \mathrm{E}-07 \\
6.1 \mathrm{E}-07 \\
3.7 \mathrm{E}-07 \\
3.2 \mathrm{E}-07 \\
3.5 \mathrm{E}-07 \\
5.0 \mathrm{E}-07 \\
6.6 \mathrm{E}-07 \\
7.6 \mathrm{E}-07 \\
9.3 \mathrm{E}-07 \\
9.4 \mathrm{E}-07 \\
9.9 \mathrm{E}-07 \\
8.6 \mathrm{E}-07\end{array}$ & $\begin{array}{l}3.1 \mathrm{E}-07 \\
2.1 \mathrm{E}-07 \\
1.8 \mathrm{E}-07 \\
1.8 \mathrm{E}-07 \\
2.9 \mathrm{E}-07 \\
3.4 \mathrm{E}-07 \\
2.0 \mathrm{E}-07 \\
1.8 \mathrm{E}-07 \\
2.0 \mathrm{E}-07 \\
2.8 \mathrm{E}-07 \\
3.7 \mathrm{E}-07 \\
4.3 \mathrm{E}-07 \\
5.2 \mathrm{E}-07 \\
5.3 \mathrm{E}-07 \\
5.6 \mathrm{E}-07 \\
4.9 \mathrm{E}-07\end{array}$ & $\begin{array}{l}3.0 \mathrm{E}-07 \\
2.0 \mathrm{E}-07 \\
1.8 \mathrm{E}-07 \\
1.7 \mathrm{E}-07 \\
2.8 \mathrm{E}-07 \\
3.3 \mathrm{E}-07 \\
1.9 \mathrm{E}-07 \\
1.7 \mathrm{E}-07 \\
1.9 \mathrm{E}-07 \\
2.7 \mathrm{E}-07 \\
3.6 \mathrm{E}-07 \\
4.2 \mathrm{E}-07 \\
5.1 \mathrm{E}-07 \\
5.1 \mathrm{E}-07 \\
5.5 \mathrm{E}-07 \\
4.8 \mathrm{E}-07\end{array}$ & $\begin{array}{l}2.0 \mathrm{E}-07 \\
1.4 \mathrm{E}-07 \\
1.2 \mathrm{E}-07 \\
1.2 \mathrm{E}-07 \\
1.8 \mathrm{E}-07 \\
2.2 \mathrm{E}-07 \\
1.3 \mathrm{E}-07 \\
1.1 \mathrm{E}-07 \\
1.3 \mathrm{E}-07 \\
1.8 \mathrm{E}-07 \\
2 \cdot 4 \mathrm{E}-07 \\
2.8 \mathrm{E}-07 \\
3.3 \mathrm{E}-07 \\
3 \cdot 4 \mathrm{E}-07 \\
3.6 \mathrm{E}-07 \\
3.2 \mathrm{E}-07\end{array}$ & $\begin{array}{l}1.5 \mathrm{E}-07 \\
1.0 \mathrm{E}-07 \\
9.1 \mathrm{E}-08 \\
9.0 \mathrm{E}-08 \\
1.4 \mathrm{E}-07 \\
1.7 \mathrm{E}-07 \\
9.6 \mathrm{E}-08 \\
8.7 \mathrm{E}-08 \\
9.7 \mathrm{E}-08 \\
1.4 \mathrm{E}-07 \\
1.8 \mathrm{E}-07 \\
2.1 \mathrm{E}-07 \\
2.5 \mathrm{E}-07 \\
2.6 \mathrm{E}-07 \\
2.8 \mathrm{E}-07 \\
2.4 \mathrm{E}-07\end{array}$ & $\begin{array}{l}1.5 \mathrm{E}-07 \\
1.0 \mathrm{E}-07 \\
8.8 \mathrm{E}-08 \\
8.8 \mathrm{E}-08 \\
1.3 \mathrm{E}-07 \\
1.6 \mathrm{E}-07 \\
9.3 \mathrm{E}-08 \\
8.4 \mathrm{E}-08 \\
9.4 \mathrm{E}-08 \\
1.3 \mathrm{E}-07 \\
1.7 \mathrm{E}-07 \\
2.1 \mathrm{E}-07 \\
2.5 \mathrm{E}-07 \\
2.5 \mathrm{E}-07 \\
2.7 \mathrm{E}-07 \\
2.3 \mathrm{E}-07\end{array}$ \\
\hline
\end{tabular}

Distance (m)

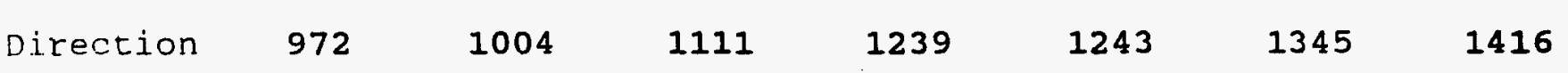

\begin{tabular}{rlllllll}
\hline & $1.3 \mathrm{E}-07$ & $1.2 \mathrm{E}-07$ & $1.0 \mathrm{E}-07$ & $8.6 \mathrm{E}-08$ & $8.5 \mathrm{E}-08$ & $7.5 \mathrm{E}-08$ & $6.9 \mathrm{E}-08$ \\
NNW & $8.8 \mathrm{E}-08$ & $8.4 \mathrm{E}-08$ & $7.1 \mathrm{E}-08$ & $6.0 \mathrm{E}-08$ & $5.9 \mathrm{E}-08$ & $5.2 \mathrm{E}-08$ & $4.8 \mathrm{E}-08$ \\
NW & $7.6 \mathrm{E}-08$ & $7.2 \mathrm{E}-08$ & $6.2 \mathrm{E}-08$ & $5.2 \mathrm{E}-08$ & $5.1 \mathrm{E}-08$ & $4.5 \mathrm{E}-08$ & $4.1 \mathrm{E}-08$ \\
WNW & $7.6 \mathrm{E}-08$ & $7.2 \mathrm{E}-08$ & $6.1 \mathrm{E}-08$ & $5.2 \mathrm{E}-08$ & $5.1 \mathrm{E}-08$ & $4.5 \mathrm{E}-08$ & $4.2 \mathrm{E}-08$ \\
$\mathrm{~N}$ & $1.2 \mathrm{E}-07$ & $1.1 \mathrm{E}-07$ & $9.4 \mathrm{E}-08$ & $7.9 \mathrm{E}-08$ & $7.8 \mathrm{E}-08$ & $6.9 \mathrm{E}-08$ & $6.3 \mathrm{E}-08$ \\
WSW & $1.4 \mathrm{E}-07$ & $1.3 \mathrm{E}-07$ & $1.1 \mathrm{E}-07$ & $9.4 \mathrm{E}-08$ & $9.4 \mathrm{E}-08$ & $8.2 \mathrm{E}-08$ & $7.6 \mathrm{E}-08$ \\
SW & $8.1 \mathrm{E}-08$ & $7.6 \mathrm{E}-08$ & $6.5 \mathrm{E}-08$ & $5.4 \mathrm{E}-08$ & $5.4 \mathrm{E}-08$ & $4.8 \mathrm{E}-08$ & $4.4 \mathrm{E}-08$ \\
SSW & $7.3 \mathrm{E}-08$ & $6.9 \mathrm{E}-08$ & $5.9 \mathrm{E}-08$ & $5.0 \mathrm{E}-08$ & $4.9 \mathrm{E}-08$ & $4.3 \mathrm{E}-08$ & $4.0 \mathrm{E}-08$ \\
$\mathrm{~S}$ & $8.1 \mathrm{E}-08$ & $7.7 \mathrm{E}-08$ & $6.6 \mathrm{E}-08$ & $5.5 \mathrm{E}-08$ & $5.5 \mathrm{E}-08$ & $4.8 \mathrm{E}-08$ & $4.4 \mathrm{E}-08$ \\
SSE & $1.1 \mathrm{E}-07$ & $1.1 \mathrm{E}-07$ & $9.2 \mathrm{E}-08$ & $7.7 \mathrm{E}-08$ & $7.7 \mathrm{E}-08$ & $6.7 \mathrm{E}-08$ & $6.2 \mathrm{E}-08$ \\
SE & $1.5 \mathrm{E}-07$ & $1.4 \mathrm{E}-07$ & $1.2 \mathrm{E}-07$ & $1.0 \mathrm{E}-07$ & $1.0 \mathrm{E}-07$ & $8.9 \mathrm{E}-08$ & $8.2 \mathrm{E}-08$ \\
$\mathrm{ESE}$ & $1.8 \mathrm{E}-07$ & $1.7 \mathrm{E}-07$ & $1.4 \mathrm{E}-07$ & $1.2 \mathrm{E}-07$ & $1.2 \mathrm{E}-07$ & $1.1 \mathrm{E}-07$ & $9.7 \mathrm{E}-08$ \\
$\mathrm{E}$ & $2.1 \mathrm{E}-07$ & $2.0 \mathrm{E}-07$ & $1.7 \mathrm{E}-07$ & $1.4 \mathrm{E}-07$ & $1.4 \mathrm{E}-07$ & $1.3 \mathrm{E}-07$ & $1.2 \mathrm{E}-07$ \\
$\mathrm{ENE}$ & $2.2 \mathrm{E}-07$ & $2.1 \mathrm{E}-07$ & $1.7 \mathrm{E}-07$ & $1.5 \mathrm{E}-07$ & $1.5 \mathrm{E}-07$ & $1.3 \mathrm{E}-07$ & $1.2 \mathrm{E}-07$ \\
$N E$ & $2.3 \mathrm{E}-07$ & $2.2 \mathrm{E}-07$ & $1.9 \mathrm{E}-07$ & $1.6 \mathrm{E}-07$ & $1.6 \mathrm{E}-07$ & $1.4 \mathrm{E}-07$ & $1.3 \mathrm{E}-07$ \\
$N N F$ & $2.0 \mathrm{E}-07$ & $1.9 \mathrm{E}-07$ & $1.6 \mathrm{E}-07$ & $1.4 \mathrm{E}-07$ & $1.4 \mathrm{E}-07$ & $1.2 \mathrm{E}-07$ & $1.1 \mathrm{E}-07$
\end{tabular}


nes 15, 1997,02:10 pm

INDIVIDUAL LIFETIME RISK (deaths)

(All Radionuclides and Pathways)

\section{Distance (m)}

nirertion 1553

\begin{tabular}{rl}
\hline$N$ & $5.9 E-08$ \\
$N N W$ & $4.1 \mathrm{E}-08$ \\
$N W$ & $3.6 \mathrm{E}-08$ \\
$W N W$ & $3.6 \mathrm{E}-08$ \\
$W$ & $5.4 \mathrm{E}-08$ \\
$W S W$ & $6.5 \mathrm{E}-08$ \\
$S W$ & $3.8 \mathrm{E}-08$ \\
$\mathrm{SSW}$ & $3.4 \mathrm{E}-08$ \\
$\mathrm{~S}$ & $3.8 \mathrm{E}-08$ \\
$\therefore S E$ & $5.3 \mathrm{E}-08$ \\
$S E$ & $7.0 \mathrm{E}-08$ \\
$\mathrm{ESE}$ & $8.3 \mathrm{E}-08$ \\
$\mathrm{E}$ & $9.9 \mathrm{E}-08$ \\
$\mathrm{ENE}$ & $1.0 \mathrm{E}-07$ \\
$N E$ & $1.1 \mathrm{E}-07$ \\
$N N E$ & $9.5 \mathrm{E}-08$ \\
\hline
\end{tabular}


ATTACHMENT A - DATASET \#3,

CAP88-PC v1.0 and v2.0

SYNOPSIS and SUMMARY FILES 
$C A P 88-P C$

Version 1.00

Clean Air Act Assessment Package - 1988

\author{
$S Y N O P S I S \quad R E P O R T$ \\ Non-Radon Population Assessment \\ Dec 15,1997 10:43 am
}

Facility: FERNALD ENVIRONMENTAL MANAGEMENT PROJECT

Address: P.0. BOX 398704

7400 WILLEY ROAD

City: CINCINNATI

State: $\mathrm{OH} \quad$ Zip: 45253-8704 


\section{Effective Dose Equivalent (mrem/year) \\ $3.46 E+02$}

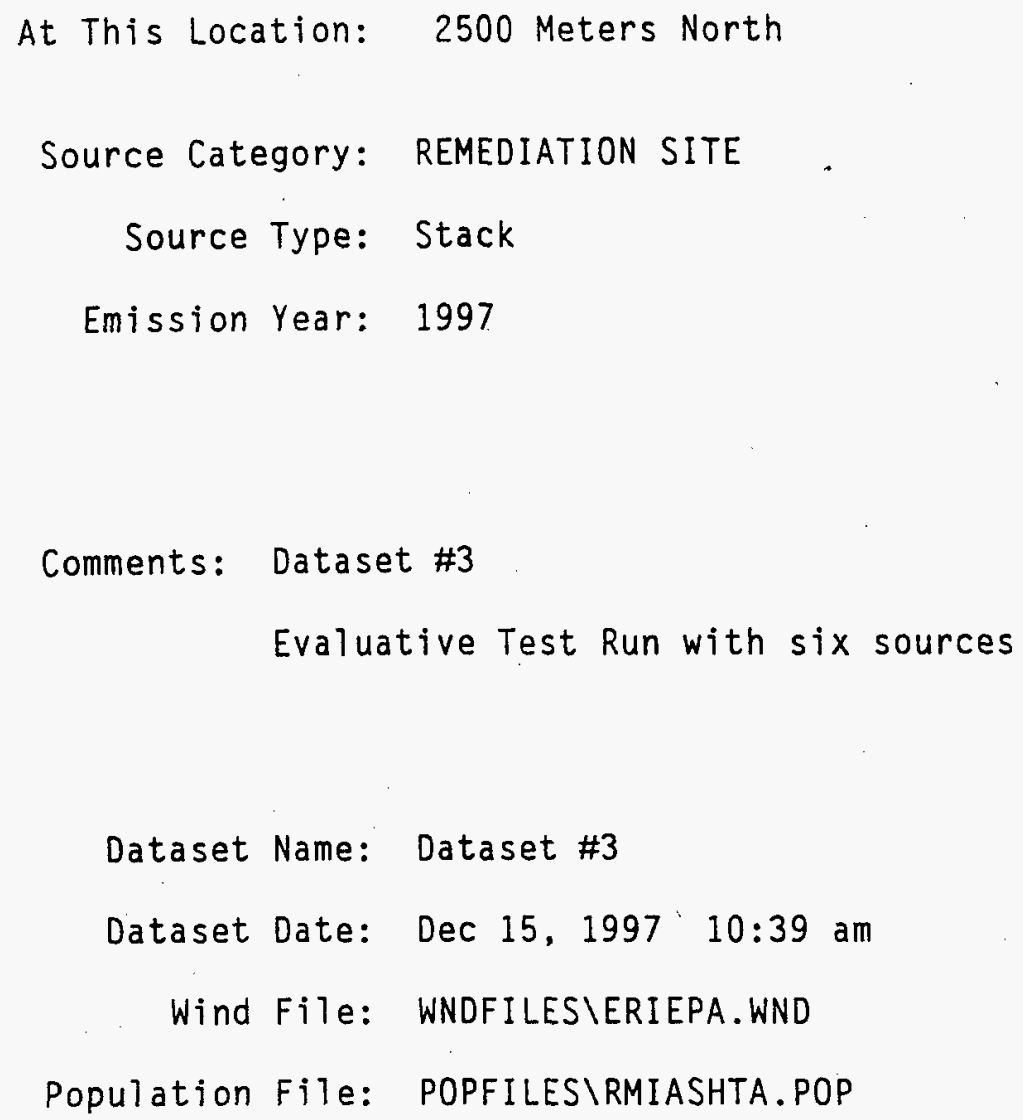


C $15,1997 \quad 10: 43$ am

NOPSIS

Page 1

MAXIMALLY EXPOSED INDIVIDUAL

Location of The Individual: 2500 Meters North

Lifetime Fatal Cancer Risk: $3.24 \mathrm{E}-03$

ORGAN DOSE EQUIVALENT SUMMARY

\begin{tabular}{lcc} 
Organ & $\begin{array}{c}\text { Selected } \\
\text { Individual } \\
\text { (mrem/y) }\end{array}$ & $\begin{array}{c}\text { Collective } \\
\text { Population } \\
\text { (person-rem/y) }\end{array}$ \\
\cline { 2 - 2 } GONADS & $2.06 E+01$ & $3.60 E+02$ \\
BREAST & $3.36 E+00$ & $5.56 E+01$ \\
R MAR & $2.72 E+02$ & $4.35 E+03$ \\
LUNGS & $1.54 E+03$ & $2.15 E+04$ \\
THYROID & $3.01 E+00$ & $5.02 E+01$ \\
ENDOST & $3.37 E+03$ & $5.39 E+04$ \\
RMNDR & $6.89 E+01$ & $1.23 E+03$ \\
EFFEC & $3.46 E+02$ & $5.19 E+03$
\end{tabular}

FREQUENCY DISTRIBUTION OF LIFETIME FATAL CANCER RISKS igher

Risk Range

$\begin{array}{cccc}\text { Number of } & \text { Number of People } & \text { Deaths/Year } & \text { Deaths/Year } \\ \text { People This Risk } & \text { In This } & \text { In This Risk } \\ \text { Range Or Higher } & \text { Risk Range } & \text { Range Or }\end{array}$

$\therefore .0 E+00$ TO $1.0 E-01$

0
0
3038
64673
1305208
27255
0

0
0
3038
67711
1372919
1400174
1400174

$0.00 E+00$

$0.00 E+00$

$0.00 \mathrm{E}+00$

$8.97 E-02$

$3.40 E-01$

$6.68 E-01$

$6.71 E-01$

6.71E-01 
Dec 15, 1997 10:43 am

SYNOPSIS

Page 2

RADIONUCLIDE EMISSIONS DURING THE YEAR 1997

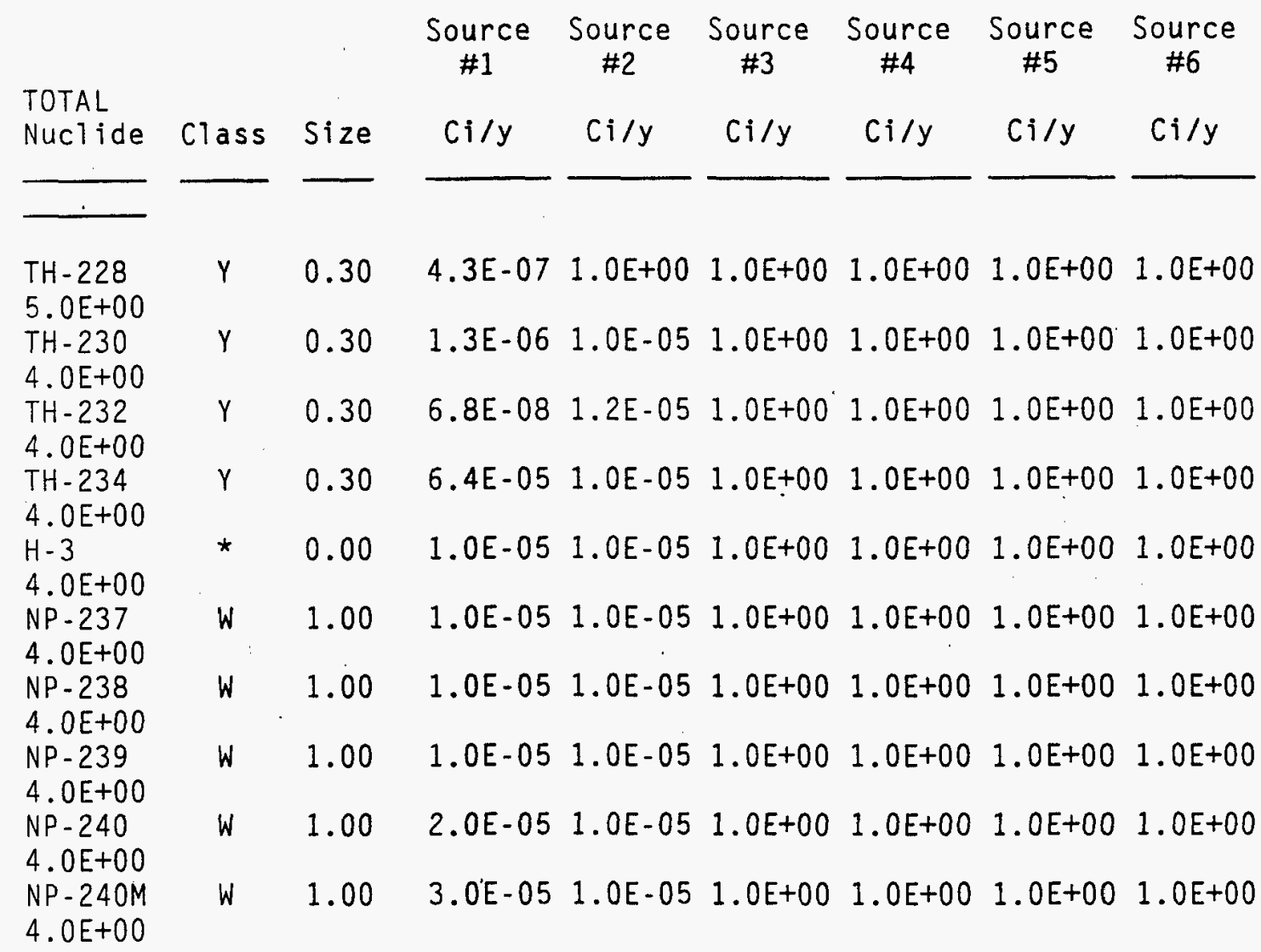

SITE INFORMATION

Temperature: 9 degrees $C$

Precipitation: $\quad 150 \mathrm{~cm} / \mathrm{y}$

Mixing Height: $\quad 950 \mathrm{~m}$

$\mathrm{Ci} / \mathrm{y}$ 


\section{SOURCE INFORMATION}

\begin{tabular}{|c|c|c|c|c|c|c|}
\hline Source Number: & 1 & 2 & 3 & 4 & 5 & 6 \\
\hline $\begin{array}{r}\text { Stack Height }(m): \\
\text { Diameter }(m):\end{array}$ & $\begin{array}{l}8.00 \\
6.00\end{array}$ & $\begin{array}{l}9.00 \\
6.00\end{array}$ & $\begin{array}{r}10.00 \\
5.00\end{array}$ & $\begin{array}{l}9.00 \\
4.00\end{array}$ & $\begin{array}{l}7.00 \\
1.00\end{array}$ & $\begin{array}{r}20.00 \\
1.00\end{array}$ \\
\hline $\begin{array}{l}\text { Plume Rise } \\
\text { Momentum }(\mathrm{m} / \mathrm{s}) \text { : } \\
\text { (Exit Velocity) }\end{array}$ & $1.23 E+02$ & $1.30 E+02$ & $1.20 E+02$ & $1.00 E+02$ & $1.50 E+02$ & $1.65 \mathrm{E}+02$ \\
\hline
\end{tabular}

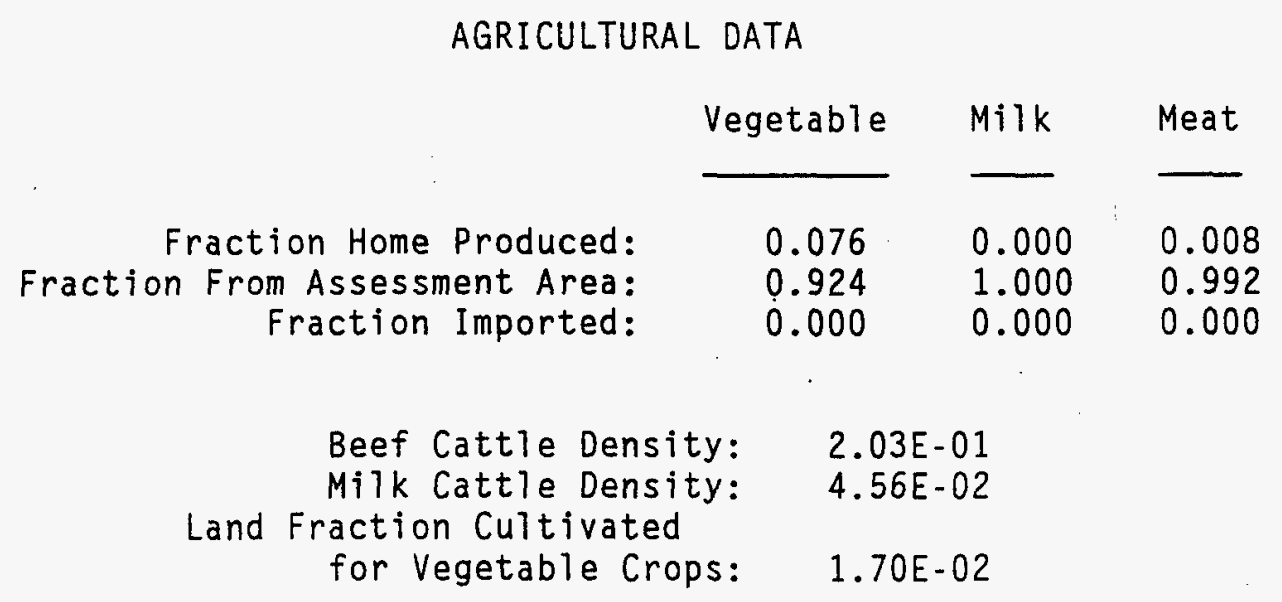


Dec 15, 1997 10:43 am

SYNOPSIS

Page 4

POPULATION DATA

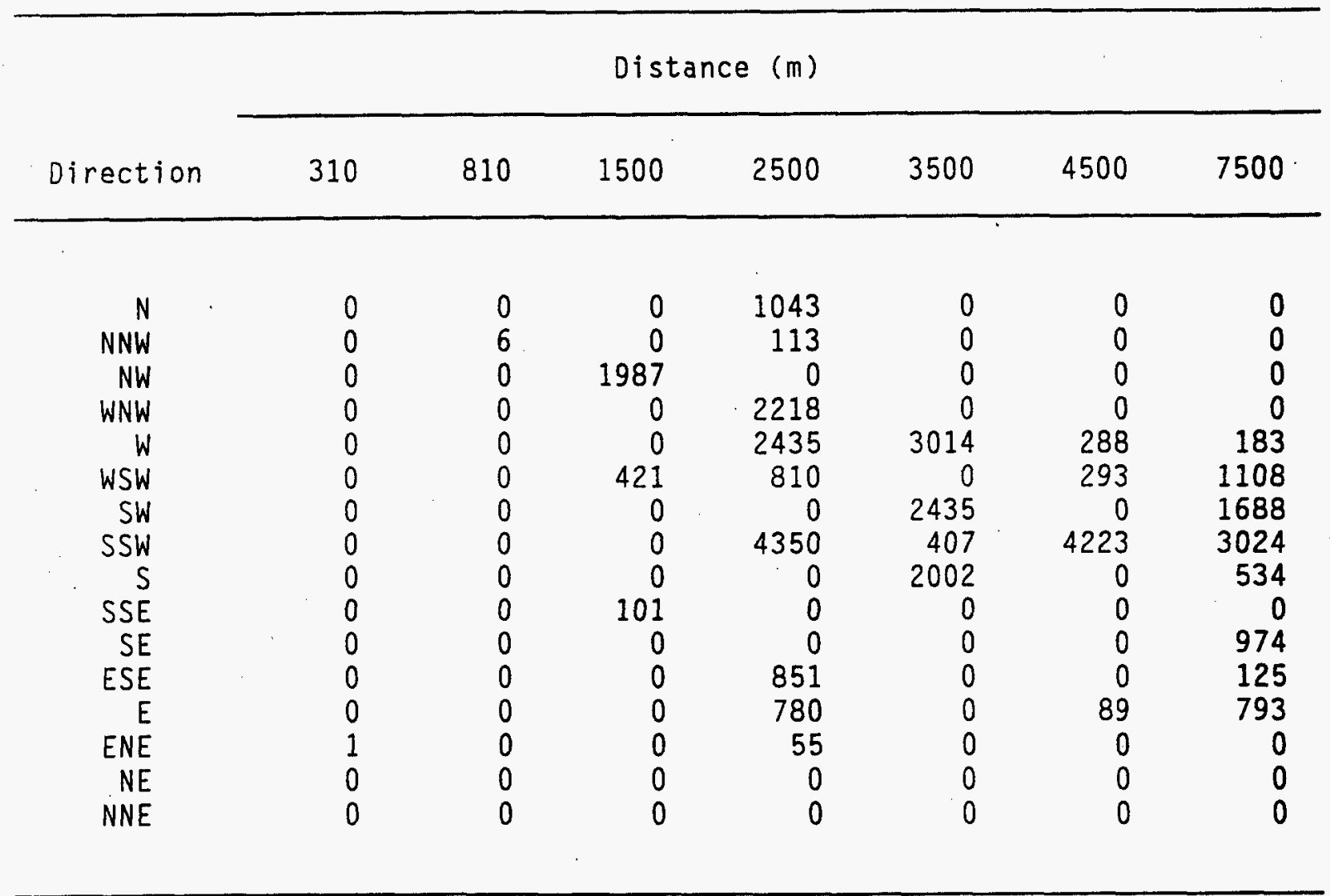

Distance (m)

\begin{tabular}{lllllll}
\cline { 2 - 3 } Direction & 15000 & 25000 & 35000 & 45000 & 55000 & 70000 \\
\hline
\end{tabular}

$\begin{array}{rrrrrrr}N & 0 & 0 & 0 & 0 & 0 & 0 \\ \text { NNW } & 0 & 0 & 0 & 0 & 0 & 0 \\ \text { NW } & 0 & 0 & 0 & 0 & 0 & 0 \\ \text { WNW } & 0 & 0 & 0 & 0 & 0 & 0 \\ W & 380 & 0 & 0 & 0 & 0 & 0 \\ W S W & 779 & 15354 & 8786 & 48943 & 63357 & 319944 \\ \text { SW } & 3376 & 3448 & 7591 & 6999 & 23331 & 187240 \\ \text { SSW } & 3059 & 3396 & 3128 & 6847 & 8940 & 27255 \\ \text { S } & 8224 & 2252 & 2388 & 5752 & 7858 & 140074 \\ \text { SSE } & 984 & 1792 & 3659 & 3759 & 7416 & 80933 \\ \text { SE } & 1388 & 2021 & 3148 & 5123 & 4351 & 14935 \\ \text { ESE } & 3161 & 827 & 3252 & 3712 & 25127 & 15531 \\ E & 265 & 1693 & 5397 & 1338 & 11236 & 17820 \\ \text { ENE } & 14641 & 6332 & 5986 & 13324 & 45833 & 150138 \\ \text { NE } & 0 & 0 & 0 & 0 & 0 & 0 \\ \text { NNE } & 0 & 0 & 0 & 0 & 0 & 0\end{array}$




\author{
C A P $88-P C$ \\ Version 1.00 \\ Clean Air Act Assessment Package - 1988
}

DOSE AND RISK EQUIVALENT SUMMARIES Non-Radon Population Assessment Dec 15, 1997 10:43 am

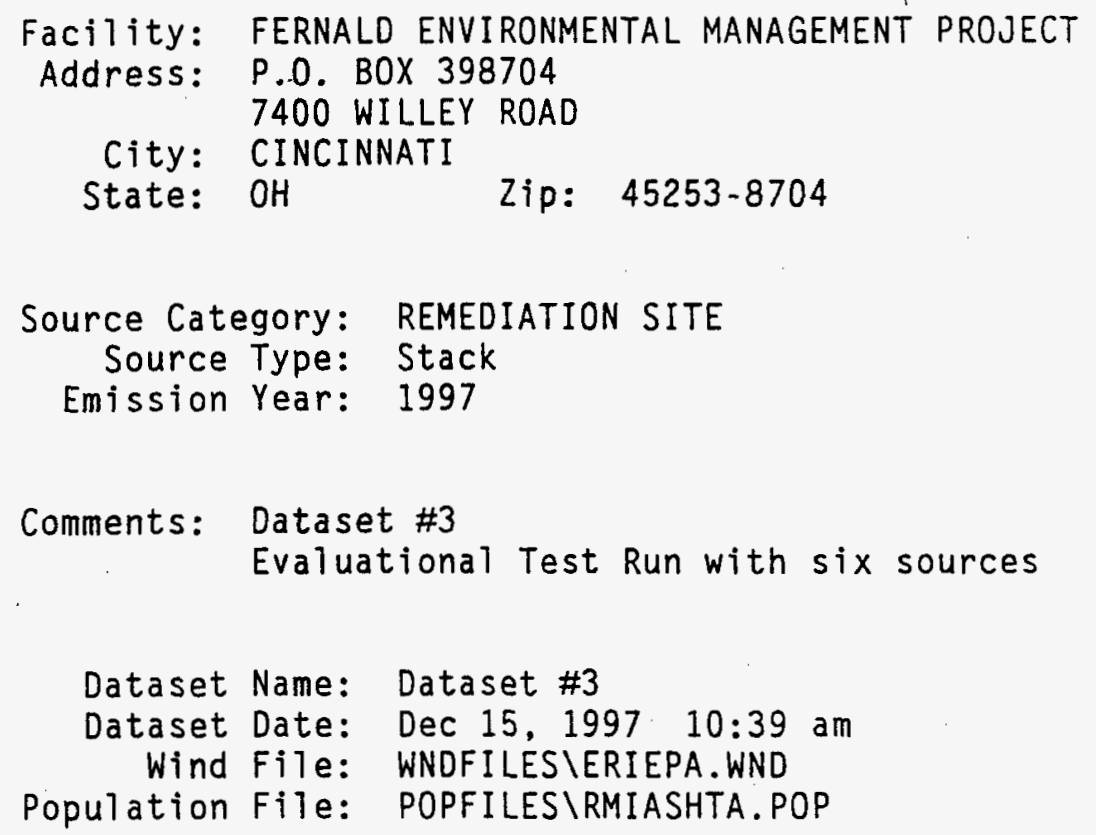




\begin{tabular}{lcc} 
Organ & $\begin{array}{c}\text { Selected } \\
\text { Individual } \\
\text { (mrem/y) }\end{array}$ & $\begin{array}{c}\text { Collective } \\
\text { Population } \\
\text { (person-rem/y) }\end{array}$ \\
\cline { 2 - 2 } GONADS & $2.06 E+01$ & $3.60 E+02$ \\
BREAST & $3.36 E+00$ & $5.56 E+01$ \\
R MAR & $2.72 E+02$ & $4.35 E+03$ \\
LUNGS & $1.54 E+03$ & $2.15 E+04$ \\
THYROID & $3.01 E+00$ & $5.02 E+01$ \\
ENDOST & $3.37 E+03$ & $5.39 E+04$ \\
RMNDR & $6.89 E+01$ & $1.23 E+03$ \\
EFFEC & $3.46 E+02$ & $5.19 E+03$
\end{tabular}

PATHWAY EFFECTIVE DOSE EQUIVALENT SUMMARY

Pathway

\begin{tabular}{l}
\hline \\
INGESTION \\
INHALATION \\
AIR IMMERSION \\
GROUND SURFACE \\
INTERNAL \\
EXTERNAL
\end{tabular}

TOTAL

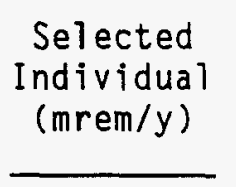

2. $35 \mathrm{E}+00$

$3.43 E+02$

2. $19 \mathrm{E}-04$

$3.44 \mathrm{E}-01$

$3.45 E+02$

$3.45 \mathrm{E}-01$

$3.46 \mathrm{E}+02$
Collective

Population (person-rem/y)

$4.86 E+02$

$4.69 E+03$

2. $10 \mathrm{E}-03$

$5.20 E+00$

$5.18 \mathrm{E}+03$

$5.20 E+00$

$5.18 E+03$ 
NUCLIDE EFFECTIVE DOSE EQUIVALENT SUMMARY

\begin{tabular}{lcc} 
Nuclides & $\begin{array}{c}\text { Selected } \\
\text { Individual } \\
\text { (mrem/y) }\end{array}$ & $\begin{array}{c}\text { Collective } \\
\text { Population } \\
\text { person-rem/y) }\end{array}$ \\
\hline TH-228 & $7.70 E+01$ & $1.15 E+03$ \\
TH-230 & $7.38 E+01$ & $1.03 E+03$ \\
TH-232 & $1.07 E+02$ & $1.48 E+03$ \\
TH-234 & $1.22 E-02$ & $6.66 E-01$ \\
$H-3$ & $3.01 E-05$ & $2.70 E-03$ \\
NP-237 & $8.74 E+01$ & $1.53 E+03$ \\
NP-238 & $6.71 E-03$ & $9.02 E-02$ \\
NP-239 & $9.07 E-04$ & $1.33 E-02$ \\
NP-240 & $1.82 E-04$ & $1.43 E-03$ \\
NP-240M & $2.06 E-05$ & $1.02 E-04$ \\
TOTAL & $3.46 E+02$ & $5.18 E+03$
\end{tabular}




\section{CANCER RISK SUMMARY}

\begin{tabular}{lcc} 
Cancer & $\begin{array}{c}\text { Selected Individual } \\
\text { Total Lifetime } \\
\text { Fatal Cancer Risk }\end{array}$ & $\begin{array}{c}\text { Total Collective } \\
\text { Population Fatal } \\
\text { Cancer Risk } \\
\text { (Deaths/y) }\end{array}$ \\
\cline { 2 - 3 } LEUKEMIA & $2.32 E-04$ & $5.26 E-02$ \\
BONE & $1.51 E-04$ & $3.42 E-02$ \\
THYROID & $4.85 E-07$ & $1.12 E-04$ \\
BREAST & $5.09 E-06$ & $1.14 E-03$ \\
LUNG & $2.61 E-03$ & $5.22 E-01$ \\
STOMACH & $2.69 E-06$ & $6.52 E-04$ \\
BOWEL & $1.92 E-06$ & $9.47 E-04$ \\
LIVER & $2.35 E-04$ & $5.81 E-02$ \\
PANCREAS & $1.88 E-06$ & $4.36 E-04$ \\
URINARY & $1.12 E-06$ & $2.58 E-04$ \\
OTHER & $2.30 E-06$ & $5.33 E-04$ \\
TOTAL & $3.24 E-03$ & $6.71 E-01$
\end{tabular}

\section{PATHWAY RISK SUMMARY}

Pathway

INGESTION

I NHA LATION

AIR IMMERSION GROUND SURFACE

INTERNAL

EXTERNAL

TOTAL

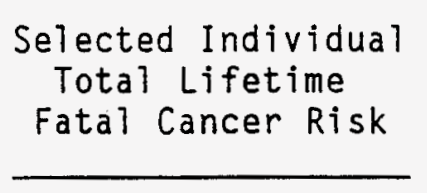

$1.05 E-05$

$3.22 E-03$

$5.22 \mathrm{E}-09$

$7.52 \mathrm{E}-06$

$3.23 \mathrm{E}-03$

$7.52 E-06$

$3.24 E-03$
Total. Collective Population Fatal Cancer Risk (Deaths/y)

3. $08 E-02$

$6.38 \mathrm{E}-01$

$7.07 \mathrm{E}-07$

$1.60 E-03$

$6.69 \mathrm{E}-01$

$1.60 \mathrm{E}-03$

$6.71 E-01$ 
PATHWAY GENETIC RISK SUMMARY

(Collective Population)

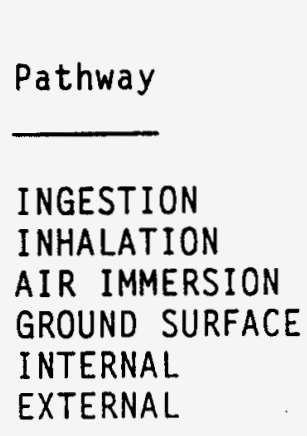

TOTAL

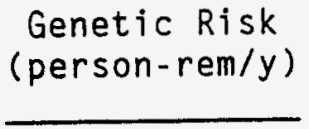

$3.75 E+00$

1. $14 \mathrm{E}+01$

$2.05 \mathrm{E}-03$

$4.37 E+00$

1. $52 \mathrm{E}+01$

$4.38 E+00$

$1.96 \mathrm{E}+01$ 


\section{NUCLIDE RISK SUMMARY}

\begin{tabular}{lc} 
Nuclide & $\begin{array}{c}\text { Selected Individual } \\
\text { Total Lifetime } \\
\text { Fatal Cancer Risk }\end{array}$ \\
\hline TH-228 & $1.56 E-03$ \\
TH-230 & $6.21 E-04$ \\
TH-232 & $6.13 E-04$ \\
TH-234 & $6.49 E-07$ \\
H-3 & $8.22 E-10$ \\
NP-237 & $4.47 E-04$ \\
NP-238 & $6.98 E-08$ \\
NP-239 & $2.57 E-08$ \\
NP-240 & $4.50 E-09$ \\
NP-240M & $5.03 E-10$ \\
TOTAL & $3.24 E-03$
\end{tabular}

Total Collective

Population Fatal Cancer Risk (Deaths/y)

3.25E- 01

$1.19 \mathrm{E}-01$

$1.18 \mathrm{E}-01$

$2.03 E-04$

$1.03 E-06$

$1.09 E-01$

1.36E- 05

$5.11 E-06$

$4.97 \mathrm{E}-07$

3. $50 \mathrm{E}-08$

$6.71 E-01$ 
INDIVIDUAL EFFECTIVE DOSE EQUIVALENT RATE $(\mathrm{mrem} / \mathrm{y})$ (AlT Radionuclides and Pathways)

\begin{tabular}{|c|c|c|c|c|c|c|c|}
\hline \multirow[b]{2}{*}{ Direction } & \multicolumn{7}{|c|}{ Distance $(m)$} \\
\hline & 310 & 810 & 1500 & 2500 & 3500 & 4500 & 7500 \\
\hline $\begin{array}{r}N \\
N N W \\
N W \\
W N W \\
W \\
W S W \\
S W \\
S S W \\
S \\
S S E \\
S E \\
E S E \\
E \\
E N E \\
N E \\
N N E\end{array}$ & $\begin{array}{l}0.0 E+00 \\
0.0 E+00 \\
0.0 E+00 \\
0.0 E+00 \\
0.0 E+00 \\
0.0 E+00 \\
0.0 E+00 \\
0.0 E+00 \\
0.0 E+00 \\
0.0 E+00 \\
0.0 E+00 \\
0.0 E+00 \\
0.0 E+00 \\
4.4 E+01 \\
0.0 E+00 \\
0.0 E+00\end{array}$ & $\begin{array}{l}0.0 E+00 \\
2.2 E+02 \\
0.0 E+00 \\
0.0 E+00 \\
0.0 E+00 \\
0.0 E+00 \\
0.0 E+00 \\
0.0 E+00 \\
0.0 E+00 \\
0.0 E+00 \\
0.0 E+00 \\
0.0 E+00 \\
0.0 E+00 \\
0.0 E+00 \\
0.0 E+00 \\
0.0 E+00\end{array}$ & $\begin{array}{l}0.0 E+00 \\
0.0 E+00 \\
6.1 E+01 \\
0.0 E+00 \\
0.0 E+00 \\
1.0 E+02 \\
0.0 E+00 \\
0.0 E+00 \\
0.0 E+00 \\
1.3 E+02 \\
0.0 E+00 \\
0.0 E+00 \\
0.0 E+00 \\
0.0 E+00 \\
0.0 E+00 \\
0.0 E+00\end{array}$ & $\begin{array}{l}3.5 E+02 \\
1.1 E+02 \\
0.0 E+00 \\
2.4 E+01 \\
3.9 E+01 \\
7.3 E+01 \\
0.0 E+00 \\
5.9 E+01 \\
0.0 E+00 \\
0.0 E+00 \\
0.0 E+00 \\
1.3 E+02 \\
2.0 E+02 \\
1.4 E+02 \\
0.0 E+00 \\
0.0 E+00\end{array}$ & $\begin{array}{l}0.0 E+00 \\
0.0 E+00 \\
0.0 E+00 \\
0.0 E+00 \\
3.1 E+01 \\
0.0 E+00 \\
6.8 E+01 \\
4.2 E+01 \\
7.0 E+01 \\
0.0 E+00 \\
0.0 E+00 \\
0.0 E+00 \\
0.0 E+00 \\
0.0 E+00 \\
0.0 E+00 \\
0.0 E+00\end{array}$ & $\begin{array}{l}0.0 E+00 \\
0.0 E+00 \\
0.0 E+00 \\
0.0 E+00 \\
2.4 E+01 \\
4.2 E+01 \\
0.0 E+00 \\
3.2 E+01 \\
0.0 E+00 \\
0.0 E+00 \\
0.0 E+00 \\
0.0 E+00 \\
1.1 E+02 \\
0.0 E+00 \\
0.0 E+00 \\
0.0 E+00\end{array}$ & $\begin{array}{l}0.0 \mathrm{E}+00 \\
0.0 \mathrm{E}+00 \\
0.0 \mathrm{E}+00 \\
0.0 \mathrm{E}+00 \\
1.5 \mathrm{E}+01 \\
2.4 \mathrm{E}+01 \\
2.9 \mathrm{E}+01 \\
1.8 \mathrm{E}+01 \\
2.8 \mathrm{E}+01 \\
0.0 \mathrm{E}+00 \\
2.4 \mathrm{E}+01 \\
3.6 \mathrm{E}+01 \\
5.7 \mathrm{E}+01 \\
0.0 \mathrm{E}+00 \\
0.0 \mathrm{E}+00 \\
0.0 \mathrm{E}+00\end{array}$ \\
\hline
\end{tabular}

Distance $(m)$

\begin{tabular}{rcccccc}
\cline { 3 - 6 } Direction & 15000 & 25000 & 35000 & 45000 & 55000 & 70000 \\
\hline$N$ & $0.0 E+00$ & $0.0 E+00$ & $0.0 E+00$ & $0.0 E+00$ & $0.0 E+00$ & $0.0 E+00$ \\
$N W$ & $0.0 E+00$ & $0.0 E+00$ & $0.0 E+00$ & $0.0 E+00$ & $0.0 E+00$ & $0.0 E+00$ \\
$N W$ & $0.0 E+00$ & $0.0 E+00$ & $0.0 E+00$ & $0.0 E+00$ & $0.0 E+00$ & $0.0 E+00$ \\
WNW & $0.0 E+00$ & $0.0 E+00$ & $0.0 E+00$ & $0.0 E+00$ & $0.0 E+00$ & $0.0 E+00$ \\
$W$ & $6.8 E+00$ & $0.0 E+00$ & $0.0 E+00$ & $0.0 E+00$ & $0.0 E+00$ & $0.0 E+00$ \\
WSW & $1.0 E+01$ & $5.3 E+00$ & $3.4 E+00$ & $2.5 E+00$ & $1.9 E+00$ & $1.4 E+00$ \\
SW & $1.2 E+01$ & $6.0 E+00$ & $3.8 E+00$ & $2.7 E+00$ & $2.1 E+00$ & $1.5 E+00$ \\
SSW & $7.4 E+00$ & $3.8 E+00$ & $2.5 E+00$ & $1.8 E+00$ & $1.4 E+00$ & $1.1 E+00$ \\
$S$ & $1.2 E+01$ & $6.1 E+00$ & $3.9 E+00$ & $2.8 E+00$ & $2.1 E+00$ & $1.6 E+00$ \\
SSE & $9.5 E+00$ & $4.8 E+00$ & $3.1 E+00$ & $2.3 E+00$ & $1.8 E+00$ & $1.3 E+00$ \\
SE & $1.0 E+01$ & $5.0 E+00$ & $3.2 E+00$ & $2.3 E+00$ & $1.8 E+00$ & $1.3 E+00$ \\
$E S E$ & $1.5 E+01$ & $7.2 E+00$ & $4.6 E+00$ & $3.2 E+00$ & $2.4 E+00$ & $1.8 E+00$ \\
$E$ & $2.3 E+01$ & $1.1 E+01$ & $7.0 E+00$ & $4.9 E+00$ & $3.6 E+00$ & $2.6 E+00$ \\
$E N E$ & $1.7 E+01$ & $8.4 E+00$ & $5.3 E+00$ & $3.7 E+00$ & $2.8 E+00$ & $2.0 E+00$ \\
$N E$ & $0.0 E+00$ & $0.0 E+00$ & $0.0 E+00$ & $0.0 E+00$ & $0.0 E+00$ & $0.0 E+00$ \\
$N E$ & $0.0 E+00$ & $0.0 E+00$ & $0.0 E+00$ & $0.0 E+00$ & $0.0 E+00$ & $0.0 E+00$ \\
$N N E$ & & & & & & \\
\hline
\end{tabular}


COLLECTIVE EFFECTIVE DOSE EQUIVALENT (person rem/y)

(A11 Radionuclides and Pathways)

\begin{tabular}{|c|c|c|c|c|c|c|c|}
\hline \multirow[b]{2}{*}{ Direction } & \multicolumn{7}{|c|}{ Distance (m) } \\
\hline & 310 & 810 & 1500 & 2500 & 3500 & 4500 & 7500 \\
\hline $\begin{array}{r}N \\
N N W \\
N W \\
W N W \\
W \\
W S W \\
S W \\
S S W \\
S \\
S S E \\
S E \\
E S E \\
E \\
E N E \\
N E \\
N N E\end{array}$ & $\begin{array}{l}0.0 E+00 \\
0.0 E+00 \\
0.0 E+00 \\
0.0 E+00 \\
0.0 E+00 \\
0.0 E+00 \\
0.0 E+00 \\
0.0 E+00 \\
0.0 E+00 \\
0.0 E+00 \\
0.0 E+00 \\
0.0 E+00 \\
0.0 E+00 \\
4.4 E-02 \\
0.0 E+00 \\
0.0 E+00\end{array}$ & $\begin{array}{l}0.0 E+00 \\
1.3 E+00 \\
0.0 E+00 \\
0.0 E+00 \\
0.0 E+00 \\
0.0 E+00 \\
0.0 E+00 \\
0.0 E+00 \\
0.0 E+00 \\
0.0 E+00 \\
0.0 E+00 \\
0.0 E+00 \\
0.0 E+00 \\
0.0 E+00 \\
0.0 E+00 \\
0.0 E+00\end{array}$ & $\begin{array}{l}0.0 E+00 \\
0.0 E+00 \\
1.2 E+02 \\
0.0 E+00 \\
0.0 E+00 \\
4.4 E+01 \\
0.0 E+00 \\
0.0 E+00 \\
0.0 E+00 \\
1.3 E+01 \\
0.0 E+00 \\
0.0 E+00 \\
0.0 E+00 \\
0.0 E+00 \\
0.0 E+00 \\
0.0 E+00\end{array}$ & $\begin{array}{l}3.6 E+02 \\
1.3 E+01 \\
0.0 E+00 \\
5.3 E+01 \\
9.6 E+01 \\
5.9 E+01 \\
0.0 E+00 \\
2.6 E+02 \\
0.0 E+00 \\
0.0 E+00 \\
0.0 E+00 \\
1.1 E+02 \\
1.6 E+02 \\
7.5 E+00 \\
0.0 E+00 \\
0.0 E+00\end{array}$ & $\begin{array}{l}0.0 E+00 \\
0.0 E+00 \\
0.0 E+00 \\
0.0 E+00 \\
9.2 E+01 \\
0.0 E+00 \\
1.7 E+02 \\
1.7 E+01 \\
1.4 E+02 \\
0.0 E+00 \\
0.0 E+00 \\
0.0 E+00 \\
0.0 E+00 \\
0.0 E+00 \\
0.0 E+00 \\
0.0 E+00\end{array}$ & $\begin{array}{l}0.0 E+00 \\
0.0 E+00 \\
0.0 E+00 \\
0.0 E+00 \\
7.0 E+00 \\
1.2 E+01 \\
0.0 E+00 \\
1.3 E+02 \\
0.0 E+00 \\
0.0 E+00 \\
0.0 E+00 \\
0.0 E+00 \\
9.5 E+00 \\
0.0 E+00 \\
0.0 E+00 \\
0.0 E+00\end{array}$ & $\begin{array}{l}0.0 E+00 \\
0.0 E+00 \\
0.0 E+00 \\
0.0 E+00 \\
2.7 E+00 \\
2.7 E+01 \\
4.9 E+01 \\
5.3 E+01 \\
1.5 E+01 \\
0.0 E+00 \\
2.3 E+01 \\
4.5 E+00 \\
4.5 E+01 \\
0.0 E+00 \\
0.0 E+00 \\
0.0 E+00\end{array}$ \\
\hline
\end{tabular}

Distance $(m)$

\begin{tabular}{|c|c|c|c|c|c|c|}
\hline Direction & 15000 & 25000 & 35000 & 45000 & 55000 & 70000 \\
\hline $\begin{array}{r}N \\
N N W \\
N W \\
W N W \\
W \\
W S W \\
S W \\
S S W \\
S \\
S S E \\
S E \\
E S E \\
E \\
E N E \\
N E \\
N N E\end{array}$ & $\begin{array}{l}0.0 E+00 \\
0.0 E+00 \\
0.0 E+00 \\
0.0 E+00 \\
2.6 E+00 \\
8.1 E+00 \\
4.1 E+01 \\
2.3 E+01 \\
9.9 E+01 \\
9.3 E+00 \\
1.4 E+01 \\
4.6 E+01 \\
6.1 E+00 \\
2.5 E+02 \\
0.0 E+00 \\
0.0 E+00\end{array}$ & $\begin{array}{l}0.0 \mathrm{E}+00 \\
0.0 \mathrm{E}+00 \\
0.0 \mathrm{E}+00 \\
0.0 \mathrm{E}+00 \\
0.0 \mathrm{E}+00 \\
8.2 \mathrm{E}+01 \\
2.1 \mathrm{E}+01 \\
1.3 \mathrm{E}+01 \\
1.4 \mathrm{E}+01 \\
8.7 \mathrm{E}+00 \\
1.0 \mathrm{E}+01 \\
6.0 \mathrm{E}+00 \\
1.9 \mathrm{E}+01 \\
5.3 \mathrm{E}+01 \\
0.0 \mathrm{E}+00 \\
0.0 \mathrm{E}+00\end{array}$ & $\begin{array}{l}0.0 E+00 \\
0.0 E+00 \\
0.0 E+00 \\
0.0 E+00 \\
0.0 E+00 \\
3.0 E+01 \\
2.9 E+01 \\
7.7 E+00 \\
9.3 E+00 \\
1.1 E+01 \\
1.0 E+01 \\
1.5 E+01 \\
3.8 E+01 \\
3.2 E+01 \\
0.0 E+00 \\
0.0 E+00\end{array}$ & $\begin{array}{l}0.0 E+00 \\
0.0 E+00 \\
0.0 E+00 \\
0.0 E+00 \\
0.0 E+00 \\
1.2 E+02 \\
1.9 E+01 \\
1.2 E+01 \\
1.6 E+01 \\
8.5 E+00 \\
1.2 E+01 \\
1.2 E+01 \\
6.5 E+00 \\
5.0 E+01 \\
0.0 E+00 \\
0.0 E+00\end{array}$ & $\begin{array}{l}0.0 E+00 \\
0.0 E+00 \\
0.0 E+00 \\
0.0 E+00 \\
0.0 E+00 \\
1.2 E+02 \\
4.9 E+01 \\
1.3 E+01 \\
1.7 E+01 \\
1.3 E+01 \\
7.8 E+00 \\
6.1 E+01 \\
4.1 E+01 \\
1.3 E+02 \\
0.0 E+00 \\
0.0 E+00\end{array}$ & $\begin{array}{l}0.0 E+00 \\
0.0 E+00 \\
0.0 E+00 \\
0.0 E+00 \\
0.0 E+00 \\
4.4 E+02 \\
2.8 E+02 \\
2.9 E+01 \\
2.2 E+02 \\
1.1 E+02 \\
2.0 E+01 \\
2.7 E+01 \\
4.6 E+01 \\
3.0 E+02 \\
0.0 E+00 \\
0.0 E+00\end{array}$ \\
\hline
\end{tabular}


Dec 15, 1997 10:43 am

SUMMARY

Page 8

AVERAGE COLLECTIVE GENETIC DOSE EQUIVALENT

(person rem)

(All Radionuclides and Pathways)

\begin{tabular}{|c|c|c|c|c|c|c|c|}
\hline \multirow[b]{2}{*}{ Direction } & \multicolumn{7}{|c|}{ Distance $(\mathrm{m})$} \\
\hline & 310 & 810 & 1500 & 2500 & 3500 & 4500 & 7500 \\
\hline $\begin{array}{r}N \\
N N W \\
N W \\
W N W \\
W \\
W S W \\
S W \\
S S W \\
S \\
S S E \\
S E \\
E S E \\
E \\
E N E \\
N E \\
N N E\end{array}$ & $\begin{array}{l}0.0 E+00 \\
0.0 E+00 \\
0.0 E+00 \\
0.0 E+00 \\
0.0 E+00 \\
0.0 E+00 \\
0.0 E+00 \\
0.0 E+00 \\
0.0 E+00 \\
0.0 E+00 \\
0.0 E+00 \\
0.0 E+00 \\
0.0 E+00 \\
4.2 E-02 \\
0.0 E+00 \\
0.0 E+00\end{array}$ & $\begin{array}{l}0.0 E+00 \\
7.9 E-01 \\
0.0 E+00 \\
0.0 E+00 \\
0.0 E+00 \\
0.0 E+00 \\
0.0 E+00 \\
0.0 E+00 \\
0.0 E+00 \\
0.0 E+00 \\
0.0 E+00 \\
0.0 E+00 \\
0.0 E+00 \\
0.0 E+00 \\
0.0 E+00 \\
0.0 E+00\end{array}$ & $\begin{array}{l}0.0 E+00 \\
0.0 E+00 \\
7.2 E+01 \\
0.0 E+00 \\
0.0 E+00 \\
2.6 E+01 \\
0.0 E+00 \\
0.0 E+00 \\
0.0 E+00 \\
7.4 E+00 \\
0.0 E+00 \\
0.0 E+00 \\
0.0 E+00 \\
0.0 E+00 \\
0.0 E+00 \\
0.0 E+00\end{array}$ & $\begin{array}{l}2.1 \mathrm{E}+02 \\
7.4 \mathrm{E}+00 \\
0.0 \mathrm{E}+00 \\
3.3 \mathrm{E}+01 \\
5.8 \mathrm{E}+01 \\
3.4 \mathrm{E}+01 \\
0.0 \mathrm{E}+00 \\
1.5 \mathrm{E}+02 \\
0.0 \mathrm{E}+00 \\
0.0 \mathrm{E}+00 \\
0.0 \mathrm{E}+00 \\
6.4 \mathrm{E}+01 \\
9.0 \mathrm{E}+01 \\
4.3 \mathrm{E}+00 \\
0.0 \mathrm{E}+00 \\
0.0 \mathrm{E}+00\end{array}$ & $\begin{array}{l}0.0 E+00 \\
0.0 E+00 \\
0.0 E+00 \\
0.0 E+00 \\
5.5 E+01 \\
0.0 E+00 \\
9.5 E+01 \\
9.9 E+00 \\
8.1 E+01 \\
0.0 E+00 \\
0.0 E+00 \\
0.0 E+00 \\
0.0 E+00 \\
0.0 E+00 \\
0.0 E+00 \\
0.0 E+00\end{array}$ & $\begin{array}{l}0.0 E+00 \\
0.0 E+00 \\
0.0 E+00 \\
0.0 E+00 \\
4.2 E+00 \\
7.1 E+00 \\
0.0 E+00 \\
7.8 E+01 \\
0.0 E+00 \\
0.0 E+00 \\
0.0 E+00 \\
0.0 E+00 \\
5.4 E+00 \\
0.0 E+00 \\
0.0 E+00 \\
0.0 E+00\end{array}$ & $\begin{array}{l}0.0 E+00 \\
0.0 E+00 \\
0.0 E+00 \\
0.0 E+00 \\
1.6 E+00 \\
1.6 E+01 \\
2.8 E+01 \\
3.1 E+01 \\
8.8 E+00 \\
0.0 E+00 \\
1.4 E+01 \\
2.6 E+00 \\
2.6 E+01 \\
0.0 E+00 \\
0.0 E+00 \\
0.0 E+00\end{array}$ \\
\hline
\end{tabular}

Distance (m)

\begin{tabular}{|c|c|c|c|c|c|c|}
\hline Direction & 15000 & 25000 & 35000 & 45000 & 55000 & 70000 \\
\hline
\end{tabular}

\begin{tabular}{rllllll}
\hline$N$ & $0.0 E+00$ & $0.0 E+00$ & $0.0 E+00$ & $0.0 E+00$ & $0.0 E+00$ & $0.0 E+00$ \\
$N N W$ & $0.0 E+00$ & $0.0 E+00$ & $0.0 E+00$ & $0.0 E+00$ & $0.0 E+00$ & $0.0 E+00$ \\
$N W$ & $0.0 E+00$ & $0.0 E+00$ & $0.0 E+00$ & $0.0 E+00$ & $0.0 E+00$ & $0.0 E+00$ \\
$W N W$ & $0.0 E+00$ & $0.0 E+00$ & $0.0 E+00$ & $0.0 E+00$ & $0.0 E+00$ & $0.0 E+00$ \\
$W$ & $1.6 E+00$ & $0.0 E+00$ & $0.0 E+00$ & $0.0 E+00$ & $0.0 E+00$ & $0.0 E+00$ \\
$W W$ & $4.9 E+00$ & $5.2 E+01$ & $2.0 E+01$ & $8.7 E+01$ & $9.1 E+01$ & $3.7 E+02$ \\
SW & $2.4 E+01$ & $1.3 E+01$ & $1.9 E+01$ & $1.3 E+01$ & $3.6 E+01$ & $2.3 E+02$ \\
SSW & $1.4 E+01$ & $8.5 E+00$ & $5.5 E+00$ & $9.5 E+00$ & $1.0 E+01$ & $2.6 E+01$ \\
$S$ & $5.9 E+01$ & $8.6 E+00$ & $6.2 E+00$ & $1.1 E+01$ & $1.2 E+01$ & $1.8 E+02$ \\
$S S E$ & $5.6 E+00$ & $5.6 E+00$ & $7.8 E+00$ & $6.2 E+00$ & $1.0 E+01$ & $9.0 E+01$ \\
SE & $8.3 E+00$ & $6.5 E+00$ & $6.9 E+00$ & $8.6 E+00$ & $6.0 E+00$ & $1.7 E+01$ \\
$E S E$ & $2.7 E+01$ & $3.7 E+00$ & $9.5 E+00$ & $8.1 E+00$ & $4.4 E+01$ & $2.1 E+01$ \\
$E$ & $3.5 E+00$ & $1.1 E+01$ & $2.3 E+01$ & $4.2 E+00$ & $2.7 E+01$ & $3.3 E+01$ \\
$E N E$ & $1.4 E+02$ & $3.2 E+01$ & $2.0 E+01$ & $3.3 E+01$ & $9.0 E+01$ & $2.3 E+02$ \\
$N E$ & $0.0 E+00$ & $0.0 E+00$ & $0.0 E+00$ & $0.0 E+00$ & $0.0 E+00$ & $0.0 E+00$ \\
$N N E$ & $0.0 E+00$ & $0.0 E+00$ & $0.0 E+00$ & $0.0 E+00$ & $0.0 E+00$ & $0.0 E+00$ \\
\hline
\end{tabular}


INDIVIDUAL LIFETIME RISK (deaths)

(A) 1 Radionuclides and Pathways)

\begin{tabular}{|c|c|c|c|c|c|c|c|}
\hline \multirow[b]{2}{*}{ Direction } & \multicolumn{7}{|c|}{ Distance (m) } \\
\hline & 310 & 810 & 1500 & 2500 & 3500 & 4500 & 7500 \\
\hline $\begin{array}{r}N \\
N N W \\
N W \\
W N W \\
W \\
W S W \\
S W \\
S S W \\
S \\
S S E \\
S E \\
E S E \\
E \\
E N E \\
N E \\
N N E\end{array}$ & $\begin{array}{l}0.0 E+00 \\
0.0 E+00 \\
0.0 E+00 \\
0.0 E+00 \\
0.0 E+00 \\
0.0 E+00 \\
0.0 E+00 \\
0.0 E+00 \\
0.0 E+00 \\
0.0 E+00 \\
0.0 E+00 \\
0.0 E+00 \\
0.0 E+00 \\
4.0 E-04 \\
0.0 E+00 \\
0.0 E+00\end{array}$ & $\begin{array}{l}0.0 E+00 \\
2.1 E-03 \\
0.0 E+00 \\
0.0 E+00 \\
0.0 E+00 \\
0.0 E+00 \\
0.0 E+00 \\
0.0 E+00 \\
0.0 E+00 \\
0.0 E+00 \\
0.0 E+00 \\
0.0 E+00 \\
0.0 E+00 \\
0.0 E+00 \\
0.0 E+00 \\
0.0 E+00\end{array}$ & $\begin{array}{l}0.0 E+00 \\
0.0 E+00 \\
5.7 E-04 \\
0.0 E+00 \\
0.0 E+00 \\
9.7 E-04 \\
0.0 E+00 \\
0.0 E+00 \\
0.0 E+00 \\
1.2 E-03 \\
0.0 E+00 \\
0.0 E+00 \\
0.0 E+00 \\
0.0 E+00 \\
0.0 E+00 \\
0.0 E+00\end{array}$ & $\begin{array}{l}3.2 E-03 \\
1.1 E-03 \\
0.0 E+00 \\
2.2 E-04 \\
3.7 E-04 \\
6.8 E-04 \\
0.0 E+00 \\
5.6 E-04 \\
0.0 E+00 \\
0.0 E+00 \\
0.0 E+00 \\
1.2 E-03 \\
1.9 E-03 \\
1.3 E-03 \\
0.0 E+00 \\
0.0 E+00\end{array}$ & $\begin{array}{l}0.0 E+00 \\
0.0 E+00 \\
0.0 E+00 \\
0.0 E+00 \\
2.8 E-04 \\
0.0 E+00 \\
6.4 E-04 \\
4.0 E-04 \\
6.6 E-04 \\
0.0 E+00 \\
0.0 E+00 \\
0.0 E+00 \\
0.0 E+00 \\
0.0 E+00 \\
0.0 E+00 \\
0.0 E+00\end{array}$ & $\begin{array}{l}0.0 E+00 \\
0.0 E+00 \\
0.0 E+00 \\
0.0 E+00 \\
2.3 E-04 \\
3.9 E-04 \\
0.0 E+00 \\
3.0 E-04 \\
0.0 E+00 \\
0.0 E+00 \\
0.0 E+00 \\
0.0 E+00 \\
1.0 E-03 \\
0.0 E+00 \\
0.0 E+00 \\
0.0 E+00\end{array}$ & $\begin{array}{l}0.0 E+00 \\
0.0 E+00 \\
0.0 E+00 \\
0.0 E+00 \\
1.4 E-04 \\
2.3 E-04 \\
2.7 E-04 \\
1.7 E-04 \\
2.7 E-04 \\
0.0 E+00 \\
2.3 E-04 \\
3.5 E-04 \\
5.5 E-04 \\
0.0 E+00 \\
0.0 E+00 \\
0.0 E+00\end{array}$ \\
\hline
\end{tabular}

Distance $(m)$

\begin{tabular}{rcccccc} 
& & & & & \\
Direction & 15000 & 25000 & 35000 & 45000 & 55000 & 70000 \\
\hline$N$ & $0.0 E+00$ & $0.0 E+00$ & $0.0 E+00$ & $0.0 E+00$ & $0.0 E+00$ & $0.0 E+00$ \\
$N N W$ & $0.0 E+00$ & $0.0 E+00$ & $0.0 E+00$ & $0.0 E+00$ & $0.0 E+00$ & $0.0 E+00$ \\
$N W$ & $0.0 E+00$ & $0.0 E+00$ & $0.0 E+00$ & $0.0 E+00$ & $0.0 E+00$ & $0.0 E+00$ \\
WNW & $0.0 E+00$ & $0.0 E+00$ & $0.0 E+00$ & $0.0 E+00$ & $0.0 E+00$ & $0.0 E+00$ \\
$W$ & $6.3 E-05$ & $0.0 E+00$ & $0.0 E+00$ & $0.0 E+00$ & $0.0 E+00$ & $0.0 E+00$ \\
WSW & $9.8 E-05$ & $5.0 E-05$ & $3.2 E-05$ & $2.2 E-05$ & $1.7 E-05$ & $1.2 E-05$ \\
SW & $1.1 E-04$ & $5.7 E-05$ & $3.6 E-05$ & $2.5 E-05$ & $1.9 E-05$ & $1.3 E-05$ \\
SSW & $7.0 E-05$ & $3.5 E-05$ & $2.2 E-05$ & $1.6 E-05$ & $1.2 E-05$ & $8.6 E-06$ \\
S & $1.1 E-04$ & $5.7 E-05$ & $3.6 E-05$ & $2.6 E-05$ & $1.9 E-05$ & $1.4 E-05$ \\
SSE & $9.0 E-05$ & $4.5 E-05$ & $2.9 E-05$ & $2.0 E-05$ & $1.5 E-05$ & $1.1 E-05$ \\
SE & $9.5 E-05$ & $4.7 E-05$ & $3.0 E-05$ & $2.1 E-05$ & $1.6 E-05$ & $1.1 E-05$ \\
ESE & $1.4 E-04$ & $6.9 E-05$ & $4.3 E-05$ & $3.0 E-05$ & $2.2 E-05$ & $1.6 E-05$ \\
$E$ & $2.2 E-04$ & $1.1 E-04$ & $6.7 E-05$ & $4.6 E-05$ & $3.4 E-05$ & $2.4 E-05$ \\
ENE & $1.6 E-04$ & $8.0 E-05$ & $5.0 E-05$ & $3.5 E-05$ & $2.6 E-05$ & $1.8 E-05$ \\
$N E$ & $0.0 E+00$ & $0.0 E+00$ & $0.0 E+00$ & $0.0 E+00$ & $0.0 E+00$ & $0.0 E+00$ \\
$N N E$ & $0.0 E+00$ & $0.0 E+00$ & $0.0 E+00$ & $0.0 E+00$ & $0.0 E+00$ & $0.0 E+00$ \\
& & & & & & \\
\hline
\end{tabular}


COLLECTIVE FATAL CANCER RATE (deaths/y)

(A11 Radionuclides and Pathways)

\begin{tabular}{|c|c|c|c|c|c|c|c|}
\hline \multirow[b]{2}{*}{ Direction } & \multicolumn{6}{|c|}{ Distance (m) } & \multirow[b]{2}{*}{7500} \\
\hline & 310 & 810 & 1500 & 2500 & 3500 & 4500 & \\
\hline $\begin{array}{r}N \\
N N W \\
N W \\
W N W \\
W \\
W S W \\
S W \\
S S W \\
S \\
S S E \\
S E \\
E S E \\
E \\
E N E \\
N E \\
N N E\end{array}$ & $\begin{array}{l}0.0 \mathrm{E}+00 \\
0.0 \mathrm{E}+00 \\
0.0 \mathrm{E}+00 \\
0.0 \mathrm{E}+00 \\
0.0 \mathrm{E}+00 \\
0.0 \mathrm{E}+00 \\
0.0 \mathrm{E}+00 \\
0.0 \mathrm{E}+00 \\
0.0 \mathrm{E}+00 \\
0.0 \mathrm{E}+00 \\
0.0 \mathrm{E}+00 \\
0.0 \mathrm{E}+00 \\
0.0 \mathrm{E}+00 \\
5.7 \mathrm{E}-06 \\
0.0 \mathrm{E}+00 \\
0.0 \mathrm{E}+00\end{array}$ & $\begin{array}{l}0.0 E+00 \\
1.8 E-04 \\
0.0 E+00 \\
0.0 E+00 \\
0.0 E+00 \\
0.0 E+00 \\
0.0 E+00 \\
0.0 E+00 \\
0.0 E+00 \\
0.0 E+00 \\
0.0 E+00 \\
0.0 E+00 \\
0.0 E+00 \\
0.0 E+00 \\
0.0 E+00 \\
0.0 E+00\end{array}$ & $\begin{array}{l}0.0 \mathrm{E}+00 \\
0.0 \mathrm{E}+00 \\
1.6 \mathrm{E}-02 \\
0.0 \mathrm{E}+00 \\
0.0 \mathrm{E}+00 \\
5.8 \mathrm{E}-03 \\
0.0 \mathrm{E}+00 \\
0.0 \mathrm{E}+00 \\
0.0 \mathrm{E}+00 \\
1.7 \mathrm{E}-03 \\
0.0 \mathrm{E}+00 \\
0.0 \mathrm{E}+00 \\
0.0 \mathrm{E}+00 \\
0.0 \mathrm{E}+00 \\
0.0 \mathrm{E}+00 \\
0.0 \mathrm{E}+00\end{array}$ & $\begin{array}{l}4.8 \mathrm{E}-02 \\
1.7 \mathrm{E}-03 \\
0.0 \mathrm{E}+00 \\
6.9 \mathrm{E}-03 \\
1.3 \mathrm{E}-02 \\
7.8 \mathrm{E}-03 \\
0.0 \mathrm{E}+00 \\
3.4 \mathrm{E}-02 \\
0.0 \mathrm{E}+00 \\
0.0 \mathrm{E}+00 \\
0.0 \mathrm{E}+00 \\
1.5 \mathrm{E}-02 \\
2.1 \mathrm{E}-02 \\
1.0 \mathrm{E}-03 \\
0.0 \mathrm{E}+00 \\
0.0 \mathrm{E}+00\end{array}$ & $\begin{array}{l}0.0 \mathrm{E}+00 \\
0.0 \mathrm{E}+00 \\
0.0 \mathrm{E}+00 \\
0.0 \mathrm{E}+00 \\
1.2 \mathrm{E}-02 \\
0.0 \mathrm{E}+00 \\
2.2 \mathrm{E}-02 \\
2.3 \mathrm{E}-03 \\
1.9 \mathrm{E}-02 \\
0.0 \mathrm{E}+00 \\
0.0 \mathrm{E}+00 \\
0.0 \mathrm{E}+00 \\
0.0 \mathrm{E}+00 \\
0.0 \mathrm{E}+00 \\
0.0 \mathrm{E}+00 \\
0.0 \mathrm{E}+00 .\end{array}$ & $\begin{array}{l}0.0 E+00 \\
0.0 E+00 \\
0.0 E+00 \\
0.0 E+00 \\
9.2 E-04 \\
1.6 E-03 \\
0.0 E+00 \\
1.8 E-02 \\
0.0 E+00 \\
0.0 E+00 \\
0.0 E+00 \\
0.0 E+00 \\
1.3 E-03 \\
0.0 E+00 \\
0.0 E+00 \\
0.0 E+00\end{array}$ & $\begin{array}{l}0.0 E+00 \\
0.0 E+00 \\
0.0 E+00 \\
0.0 E+00 \\
3.5 E-04 \\
3.5 E-03 \\
6.5 E-03 \\
7.1 E-03 \\
2.0 E-03 \\
0.0 E+00 \\
3.1 E-03 \\
6.1 E-04 \\
6.1 E-03 \\
0.0 E+00 \\
0.0 E+00 \\
0.0 E+00\end{array}$ \\
\hline
\end{tabular}

Distance (m)

\begin{tabular}{rcccccc} 
& & & & & \\
\cline { 3 - 6 } Direction & 15000 & 25000 & 35000 & 45000 & 55000 & 70000 \\
$N$ & $0.0 E+00$ & $0.0 E+00$ & $0.0 E+00$ & $0.0 E+00$ & $0.0 E+00$ & $0.0 E+00$ \\
$N N W$ & $0.0 E+00$ & $0.0 E+00$ & $0.0 E+00$ & $0.0 E+00$ & $0.0 E+00$ & $0.0 E+00$ \\
$N W$ & $0.0 E+00$ & $0.0 E+00$ & $0.0 E+00$ & $0.0 E+00$ & $0.0 E+00$ & $0.0 E+00$ \\
WNW & $0.0 E+00$ & $0.0 E+00$ & $0.0 E+00$ & $0.0 E+00$ & $0.0 E+00$ & $0.0 E+00$ \\
$W$ & $3.4 E-04$ & $0.0 E+00$ & $0.0 E+00$ & $0.0 E+00$ & $0.0 E+00$ & $0.0 E+00$ \\
WSW & $1.1 E-03$ & $1.1 E-02$ & $3.9 E-03$ & $1.5 E-02$ & $1.5 E-02$ & $5.3 E-02$ \\
SW & $5.5 E-03$ & $2.8 E-03$ & $3.8 E-03$ & $2.5 E-03$ & $6.2 E-03$ & $3.5 E-02$ \\
SSW & $3.0 E-03$ & $1.7 E-03$ & $9.9 E-04$ & $1.5 E-03$ & $1.5 E-03$ & $3.3 E-03$ \\
$S$ & $1.3 E-02$ & $1.8 E-03$ & $1.2 E-03$ & $2.1 E-03$ & $2.1 E-03$ & $2.7 E-02$ \\
SSE & $1.3 E-03$ & $1.1 E-03$ & $1.5 E-03$ & $1.1 E-03$ & $1.6 E-03$ & $1.3 E-02$ \\
SE & $1.9 E-03$ & $1.4 E-03$ & $1.3 E-03$ & $1.5 E-03$ & $9.8 E-04$ & $2.4 E-03$ \\
ESE & $6.3 E-03$ & $8.1 E-04$ & $2.0 E-03$ & $1.6 E-03$ & $7.9 E-03$ & $3.4 E-03$ \\
$E$ & $8.3 E-04$ & $2.6 E-03$ & $5.1 E-03$ & $8.7 E-04$ & $5.4 E-03$ & $6.0 E-03$ \\
ENE & $3.4 E-02$ & $7.2 E-03$ & $4.2 E-03$ & $6.5 E-03$ & $1.7 E-02$ & $3.8 E-02$ \\
$N E$ & $0.0 E+00$ & $0.0 E+00$ & $0.0 E+00$ & $0.0 E+00$ & $0.0 E+00$ & $0.0 E+00$ \\
$N N E$ & $0.0 E+00$ & $0.0 E+00$ & $0.0 E+00$ & $0.0 E+00$ & $0.0 E+00$ & $0.0 E+00$
\end{tabular}


C A P 8 8 - P C

Version 2.00

Clean Air Act Assessment Package - 1988

$\begin{array}{lllllllllllll}S & Y & N & O & P & S & I & S & R & P & O & R & T\end{array}$

Non-Radon Population Assessment

Dec 16, 1997 01:11 pm

\footnotetext{
Facility: Fernald Environmental Management Project Address: P.O. Box 398704

7400 Willey Road

City: Cincinnati

State: OH Zip: 45253-8704
}
Source Category: Remediation Site Source Type: stack
Emission Year: 1997

Comments: Dataset \#3

Evaluative Test Run with six sources

Effective Dose Equivalent

(mrem/year)

$3.46 E+02$

At This Location: 2500 Meters North

Dataset Name: Dataset32.

Dataset Date: Dec 16, 1997 01:10 pm

Wind File: F: \CAP88PC2 $\backslash$ WNDFILES $\backslash$ ERIEPA. WND

Population File: F:|CAP88PC2 \POPFILES\RMIASHTA. POP 
Dec 16, 1997 01:11 pm

\section{MAXIMALIY EXPOSED INDIVIDUAL}

Location of The Individual: 2500 Meters North

Lifetime Fatal Cancer Risk: $3.24 \mathrm{E}-03$

ORGAN DOSE EQUIVALENT SUMMARY

\begin{tabular}{lcc} 
Organ & $\begin{array}{c}\text { Selected } \\
\text { Individual } \\
(\text { mrem/Y) }\end{array}$ & $\begin{array}{c}\text { Collective } \\
\text { Population } \\
\text { (person-rem/Y) }\end{array}$ \\
\hline GONADS & $2.06 \mathrm{E}+01$ & $3.60 \mathrm{E}+02$ \\
BREAST & $3.36 \mathrm{E}+00$ & $5.56 \mathrm{E}+01$ \\
R MAR & $2.72 \mathrm{E}+02$ & $4.35 \mathrm{E}+03$ \\
LUNGS & $1.54 \mathrm{E}+03$ & $2.15 \mathrm{E}+04$ \\
THYROID & $3.01 \mathrm{E}+00$ & $5.02 \mathrm{E}+01$ \\
ENDOST & $3.37 \mathrm{E}+03$ & $5.39 \mathrm{E}+04$ \\
RMNDR & $6.89 \mathrm{E}+01$ & $1.23 \mathrm{E}+03$ \\
EFFEC & $3.46 \mathrm{E}+02$ & $5.19 \mathrm{E}+03$
\end{tabular}

FREQUENCY DISTRIBUTION OF LIFETIME FATAL CANCER RISKS

Risk Range

$1.0 E+00$ TO $1.0 E-01$

1. OE-01 TO 1. OE-02

1. OE-02 TO $1.0 \mathrm{E}-03$

1. OE-03 TO 1. $1.0 \mathrm{E}-04$

$1.0 \mathrm{E}-04$ TO $1.0 \mathrm{E}-05$
$1.0 \mathrm{E}-05$ TO $1.0 \mathrm{E}-06$

LESS THAN $1.0 \mathrm{E}-06$
\# \# of People

\# of in This Risk

Deaths/Year in This People Range or Higher Risk Range

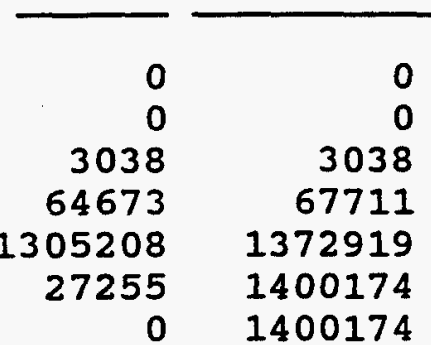

Deaths/Year in This Risk Range or Higher
$0.00 \mathrm{E}+00$

$0.00 \mathrm{E}+00$

$8.97 \mathrm{E}-02$

$3.40 \mathrm{E}-01$

$6.68 \mathrm{E}-01$

6. 71E-01

$6.71 \mathrm{E}-01$ 
RADIONUCLIDE EMISSIONS DURING THE YEAR 1997

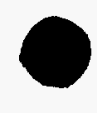

Source

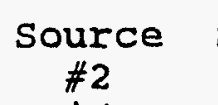

Source
$\# 3$
Ci

Source
$\# 4$
ci/y

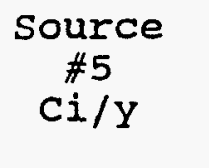

Source

Nuclide Class Size

$\mathrm{Ci} / \mathrm{Y}$ $\mathrm{Ci} / \mathrm{Y}$

$\mathrm{Ci} / \mathrm{Y}$

$\mathrm{Ci} / \mathrm{Y}$ \#6

$\mathrm{Ci} / \mathrm{Y}$

TOTAL

$\mathrm{Ci} / \mathrm{Y}$

$\mathrm{TH}-2 ? \mathrm{P}$

TH-2:0

0.30

$4.3 \mathrm{E}-07$

1. $0 E+00$

1. $O E+00$

1. $0 E+001.0 E+00$

1. $0 E+00$

1. $0 \mathrm{E}+00$ 1. $0 \mathrm{E}+00$

1. $0 E+00 \quad 5.0 E+00$

TH -232

0.30

1. $3 E-06$

$1.0 E-05$

1. $0 \mathrm{E}+00$

1. $0 \mathrm{E}+00$

1. $0 \mathrm{E}+00$

1. $0 \mathrm{E}+00$

4. $0 \mathrm{E}+00$

$\mathrm{TH}-234$

0.30

$6.8 E-081.2 E-05$

$1.0 \mathrm{E}+00$

1. $0 \mathrm{E}+00$

1. $0 \mathrm{E}+00 \quad 4.0 \mathrm{E}+00$

$\mathrm{H}-3$

$N P-237$

0.00

$6.4 E-05$

1. $0 E-05$

$1.0 E+00$

1. $0 \mathrm{E}+00$ 1. $0 \mathrm{E}+00$

1. $0 \mathrm{E}+00 \quad 4.0 \mathrm{E}+00$

$N P-238$

1.00

1. $0 E-05$

1.OE-05 1.OE+00

1. $0 \mathrm{E}+00$ 1. $0 \mathrm{E}+00$

1. $0 \mathrm{E}+00 \quad 4.0 \mathrm{E}+00$

$\mathrm{NP}-239$

1.00

1. $0 E-05$

1. OE-05 1.0E+00

1. $0 E+001.0 E+00$

1. $0 \mathrm{E}+00 \quad 4.0 \mathrm{E}+00$

$N P-240$

1.00

1. OE-05

1. OE-05 1. $0 E+00$

$1.0 \mathrm{E}+001.0 \mathrm{E}+00$

1. $0 E+00$

$4.0 \mathrm{E}+00$

$\mathrm{NP}-24 \mathrm{OM}$

$1.002 .0 E-05$
$1.003 .0 E-05$

$1.0 E-05$ 1. $0 E+00$

1. OE+00 1. $0 E+00$

$1.0 \mathrm{E}+00$

4. $0 \mathrm{E}+00$

W

$1.003 .0 \mathrm{E}-05$ 1. $0 \mathrm{E}-051.0 \mathrm{E}+00$

1. $0 E+00$

$1.0 \mathrm{E}+00$

$1.0 E+00$

4. $0 \mathrm{E}+00$

$4.0 \mathrm{E}+00$

\section{SITE INFORMATION}

Temperature: Precipitation: Mixing Height:

9 degrees C $150 \mathrm{~cm} / \mathrm{Y}$

$950 \mathrm{~m}$ 
SOURCE INFORMATION

\begin{tabular}{|c|c|c|c|c|c|c|}
\hline Source Number: & 1 & 2 & 3 & 4 & 5 & 6 \\
\hline $\begin{array}{r}\text { stack Height }(\mathrm{m}): \\
\text { Diameter }(\mathrm{m}):\end{array}$ & $\begin{array}{l}8 . \\
6 .\end{array}$ & $\begin{array}{l}9 . \\
6 .\end{array}$ & $\begin{aligned} 10 . \\
5 .\end{aligned}$ & $\begin{array}{l}9 . \\
4 .\end{array}$ & $\begin{array}{l}7 . \\
1 .\end{array}$ & $\begin{aligned} & 20 \\
& 1\end{aligned}$ \\
\hline $\begin{array}{l}\text { Plume Rise } \\
\text { Momentum (m/s): } \\
\text { (Exit Velocity) }\end{array}$ & 123 & 130 & 120. & 100 & 150 & 165 \\
\hline
\end{tabular}

AGRICULTURAI DATA

Fraction Home Produced: Fraction From Assessment Area: Vegetable Milk Meat Fraction Imported:

$\begin{array}{lll}0.076 & 0.000 & 0.008 \\ 0.924 & 1.000 & 0.992 \\ 0.000 & 0.000 & 0.000\end{array}$

Beef Cattle Density: $2.03 E-01$ Milk Cattle Density: $4.56 \mathrm{E}-02$

Land Fraction Cultivated for Vegetable Crops: $\quad 1.70 \mathrm{E}-02$ 
POPULATION DATA

\section{Distance (m)}

)ircotion 310

810

1500

2500

3500

4500

7500

$\frac{1}{2}$

NNW

Vin

WNW

WSW

$\because W$

SSW

$S$

SSE

$S E$

ESE

$E$
$E N E$

$N E$

NNE

i
$W$
$\vdots$
W
$E$
$E$
$E$
$E$
$E$

0
0
0
0
0
0
0
0
0
0
0
0
0
1
0
0

$\begin{array}{rr}0 & 0 \\ 6 & 0 \\ 0 & 1987 \\ 0 & 0 \\ 0 & 0 \\ 0 & 421 \\ 0 & 0 \\ 0 & 0 \\ 0 & 0 \\ 0 & 101 \\ 0 & 0 \\ 0 & 0 \\ 0 & 0 \\ 0 & 0 \\ 0 & 0 \\ 0 & 0\end{array}$

1043
113
0
2218
2435
810
0
4350
0
0
0
851
780
55
0
0

0

0

0

0

0

288

293

3014

2435

407

2002

4223

4223

0

0

0
0

0

89

0

0

0

0

0

0

0

1

Distance (m)

Direction $15000 \quad 25000 \quad 35000 \quad 45000 \quad 55000 \quad 70000$

$\begin{array}{rrrrrrr}N & 0 & 0 & 0 & 0 & 0 & 0 \\ N N W & 0 & 0 & 0 & 0 & 0 & 0 \\ N W & 0 & 0 & 0 & 0 & 0 & 0 \\ W N W & 0 & 0 & 0 & 0 & 0 & 0 \\ W & 380 & 0 & 0 & 0 & 0 & 0 \\ W S W & 779 & 15354 & 8786 & 48943 & 63357 & 319944 \\ S W & 3376 & 3448 & 7591 & 6999 & 23331 & 187240 \\ S S W & 3059 & 3396 & 3128 & 6847 & 8940 & 27255 \\ S & 8224 & 2252 & 2388 & 5752 & 7858 & 140074 \\ S S E & 984 & 1792 & 3659 & 3759 & 7416 & 80933 \\ S E & 1388 & 2021 & 3148 & 5123 & 4351 & 14935 \\ \text { ESE } & 3161 & 827 & 3252 & 3712 & 25127 & 15531 \\ E & 265 & 1693 & 5397 & 1338 & 11236 & 17820 \\ E N E & 14641 & 6332 & 5986 & 13324 & 45833 & 150138 \\ N E & 0 & 0 & 0 & 0 & 0 & 0 \\ N N E & 0 & 0 & 0 & 0 & 0 & 0 \\ N & & & & & 0 & \end{array}$


C A P 8 - P C

Version 2.00

Clean Air Act Assessment Package - 1988

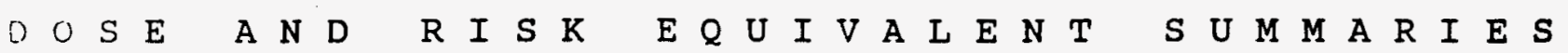

Non-Radon Population Assessment

Dec 16, 1997 01:11 pm

$\begin{aligned} & \text { Facility: Fernald Environmental Management Project } \\ & \text { Address: } \text { P.O. Box } 398704 \\ & 7400 \text { Willey Road } \\ & \text { City: Cincinnati } \\ & \text { State: OH } \text { Zip: } 45253-8704\end{aligned}$

Source Category: Remediation site

Source Type: Stack

Emission Year: 1997

Comments: Dataset \#3

Evaluative Test Run with six sources

Dataset Name: Dataset32.

Dataset Date: Dec 16,1997 01:10 pm

Wind File: F: \CAP88PC2 \WNDFILES \ERIEPA.WND

Population File: F:\CAP88PC2 \POPFILES \RMIASHTA.POP 


\begin{tabular}{lcc} 
Organ & $\begin{array}{c}\text { Selected } \\
\text { Individual } \\
\text { (mrem/y) }\end{array}$ & $\begin{array}{c}\text { Collective } \\
\text { Population } \\
\text { (person-rem/y) }\end{array}$ \\
\cline { 2 - 2 } GONADS & $2.06 \mathrm{E}+01$ & $3.60 \mathrm{E}+02$ \\
BREAST & $3.36 \mathrm{E}+00$ & $5.56 \mathrm{E}+01$ \\
R MAR & $2.72 \mathrm{E}+02$ & $4.35 \mathrm{E}+03$ \\
LUNGS & $1.54 \mathrm{E}+03$ & $2.15 \mathrm{E}+04$ \\
THYROID & $3.01 \mathrm{E}+00$ & $5.02 \mathrm{E}+01$ \\
ENDOST & $3.37 \mathrm{E}+03$ & $5.39 \mathrm{E}+04$ \\
RMNDR & $6.89 \mathrm{E}+01$ & $1.23 \mathrm{E}+03$ \\
EFFEC & $3.46 \mathrm{E}+02$ & $5.19 \mathrm{E}+03$
\end{tabular}

PATHWAY EFFECTIVE DOSE EQUIVALENT SUMMARY

\begin{tabular}{lcc} 
Pathway & $\begin{array}{c}\text { Selected } \\
\text { Individual } \\
\text { (mrem/Y) }\end{array}$ & $\begin{array}{c}\text { Collective } \\
\text { Population } \\
\text { (person-rem/y) }\end{array}$ \\
\cline { 2 - 2 } & $2.35 \mathrm{E}+00$ & \\
INGESTION & $3.43 \mathrm{E}+02$ & $4.86 \mathrm{E}+02$ \\
INHALATION & $2.19 \mathrm{E}-04$ & $4.69 \mathrm{E}+03$ \\
AIR IMMERSION & $3.44 \mathrm{E}-01$ & $2.10 \mathrm{E}-03$ \\
GROUND SURFACE & $3.45 \mathrm{E}+02$ & $5.20 \mathrm{E}+00$ \\
INTERNAL & $3.45 \mathrm{E}-01$ & $5.18 \mathrm{E}+03$ \\
EXTERNAL & $3.46 \mathrm{E}+02$ & $5.20 \mathrm{E}+00$ \\
TOTAL & & $5.18 \mathrm{E}+03$
\end{tabular}




\section{NUCLIDE EFFECTIVE DOSE EQUIVALENT SUMMARY}

\begin{tabular}{lcc} 
Nuclides & $\begin{array}{c}\text { Selected } \\
\text { Individual } \\
(\text { mrem/Y) }\end{array}$ & $\begin{array}{c}\text { Collective } \\
\text { Population } \\
\text { (person-rem } / Y \text { ) }\end{array}$ \\
\hline TH-228 & $7.70 \mathrm{E}+01$ & $1.15 \mathrm{E}+03$ \\
$\mathrm{TH}-230$ & $7.38 \mathrm{E}+01$ & $1.03 \mathrm{E}+03$ \\
$\mathrm{TH}-232$ & $1.07 \mathrm{E}+02$ & $1.48 \mathrm{E}+03$ \\
$\mathrm{TH}-234$ & $1.22 \mathrm{E}-02$ & $6.66 \mathrm{E}-01$ \\
$\mathrm{H}-3$ & $3.01 \mathrm{E}-05$ & $2.70 \mathrm{E}-03$ \\
$\mathrm{NP}-237$ & $8.74 \mathrm{E}+01$ & $1.53 \mathrm{E}+03$ \\
$\mathrm{NP}-238$ & $6.71 \mathrm{E}-03$ & $9.02 \mathrm{E}-02$ \\
$\mathrm{NP}-239$ & $9.07 \mathrm{E}-04$ & $1.33 \mathrm{E}-02$ \\
$\mathrm{NP}-240$ & $1.82 \mathrm{E}-04$ & $1.43 \mathrm{E}-03$ \\
$\mathrm{NP}-240 \mathrm{M}$ & $2.06 \mathrm{E}-05$ & $1.02 \mathrm{E}-04$ \\
TOTAL & $3.46 \mathrm{E}+02$ & $5.18 \mathrm{E}+03$
\end{tabular}


CANCER RISK SUMMARY

\begin{tabular}{|c|c|c|}
\hline Cancer & $\begin{array}{l}\text { Selected Individual } \\
\text { Total Lifetime } \\
\text { Fatal Cancer Risk }\end{array}$ & $\begin{array}{c}\text { Total Collective } \\
\text { Population Fatal } \\
\text { Cancer Risk } \\
\text { (Deaths/y) }\end{array}$ \\
\hline $\begin{array}{l}\text { LEUKEMTA } \\
\text { BONE } \\
\text { THYROID } \\
\text { BREAST } \\
\text { LUNG } \\
\text { STOMACH } \\
\text { BOWEL } \\
\text { LIVER } \\
\text { PANCREAS } \\
\text { URINARY } \\
\text { OTHER }\end{array}$ & $\begin{array}{l}2.32 \mathrm{E}-04 \\
1.51 \mathrm{E}-04 \\
4.85 \mathrm{E}-07 \\
5.09 \mathrm{E}-06 \\
2.61 \mathrm{E}-03 \\
2.69 \mathrm{E}-06 \\
1.92 \mathrm{E}-06 \\
2.35 \mathrm{E}-04 \\
1.88 \mathrm{E}-06 \\
1.12 \mathrm{E}-06 \\
2.30 \mathrm{E}-06\end{array}$ & $\begin{array}{l}5.26 \mathrm{E}-02 \\
3.42 \mathrm{E}-02 \\
1.12 \mathrm{E}-04 \\
1.14 \mathrm{E}-03 \\
5.22 \mathrm{E}-01 \\
6.52 \mathrm{E}-04 \\
9.47 \mathrm{E}-04 \\
5.81 \mathrm{E}-02 \\
4.36 \mathrm{E}-04 \\
2.58 \mathrm{E}-04 \\
5.33 \mathrm{E}-04\end{array}$ \\
\hline TOTAL & $3.24 \mathrm{E}-03$ & $6.71 \mathrm{E}-01$ \\
\hline
\end{tabular}

PATHWAY RISK SUMMARY

Pathway

Selected Individual Total Lifetime Fatal Cancer Risk

Total collective Population Fatal Cancer Risk (Deaths/Y)

INGESTION INHALATION AIR IMMERSION GROUND SURFACE INTERNAL EXTERNAL

TOTAL
1. 05E-05

3. 22E-03

5.22E-09

7. $52 \mathrm{E}-06$

$3.23 \mathrm{E}-03$

$7.52 \mathrm{E}-06$

$3.24 E-03$
$3.08 \mathrm{E}-02$

$6.38 \mathrm{E}-01$

7. 07E-07

1. $60 \mathrm{E}-03$

$6.69 \mathrm{E}-01$

1. $60 \mathrm{E}-03$

$6.71 E-01$ 


\section{PATHWAY GENETIC RISK SUMMARY}

(Collective Population)

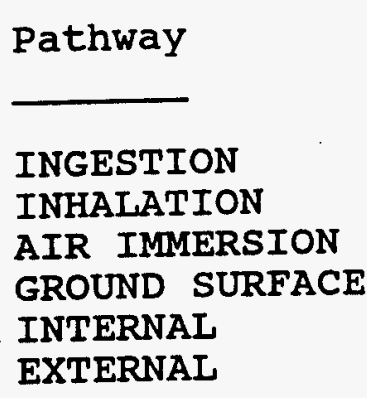

TOTAL
Genetic Risk (person-rem/Y)

3. $75 E+00$

$1.14 \mathrm{E}+01$

$2.05 \mathrm{E}-03$

$4.37 \mathrm{E}+00$

1. $52 \mathrm{E}+01$

4. $38 \mathrm{E}+00$

$1.96 \mathrm{E}+01$ 
NUCLIDE RISK SUMMARY

\section{Nuclide}

TH-228

TH-230

TH-232

TH-234

$\mathrm{H}-3$

NP -237

NP-238

NP-239

NP-2 40

NP-2 $40 M$

TOTAL
Selected Individual Total Lifetime Fatal Cancer Risk

1. 56E-03

6. 21E-04

$6.13 \mathrm{E}-04$

6. 49E-07

8. $22 \mathrm{E}-10$

$4.47 E-04$

$6.98 \mathrm{E}-08$

$2.57 \mathrm{E}-08$

4. 50E-09

5. 03E-10

$3.24 \mathrm{E}-03$
Total Collective Population Fatal Cancer Risk (Deaths/Y)

3. 25E-01

1. 19E-01

1. $18 \mathrm{E}-01$

$2.03 E-04$

1. $03 E-06$

1. 09E-01

1. 36E-05

5. 11E-06

4.97E-07

3. 50E-08

$6.71 E-01$ 
INDIVIDUAL EFFECTIVE DOSE EQUIVALENT RATE (mrem/Y) (All Radionuclides and Pathways)

Distance ( $m$ )

$\begin{array}{llllllll}\text { nirection } & 310 & 810 & 1500 & 2500 & 3500 & 4500 & 7500\end{array}$

\begin{tabular}{rlllllll}
\hline$N$ & $0.0 E+00$ & $0.0 E+00$ & $0.0 E+00$ & $3.5 E+02$ & $0.0 E+00$ & $0.0 E+00$ & $0.0 E+00$ \\
$N$ & $0.0 E+00$ & $2.2 E+02$ & $0.0 E+00$ & $1.1 E+02$ & $0.0 E+00$ & $0.0 E+00$ & $0.0 E+00$ \\
$N N W$ & $0.0 E+00$ & $0.0 E+00$ & $6.1 E+01$ & $0.0 E+00$ & $0.0 E+00$ & $0.0 E+00$ & $0.0 E+00$ \\
$N W$ & $0.0 E+00$ & $0.0 E+00$ & $0.0 E+00$ & $2.4 E+01$ & $0.0 E+00$ & $0.0 E+00$ & $0.0 E+00$ \\
$W N W$ & $0.0 E+00$ & $0.0 E+00$ & $0.0 E+00$ & $3.9 E+01$ & $3.1 E+01$ & $2.4 E+01$ & $1.5 E+01$ \\
$W$ & $0.0 E+00$ & $0.0 E+00$ & $1.0 E+02$ & $7.3 E+01$ & $0.0 E+00$ & $4.2 E+01$ & $2.4 E+01$ \\
$W S W$ & $0.0 E+00$ & $0.0 E+00$ & $0.0 E+00$ & $0.0 E+00$ & $6.8 E+01$ & $0.0 E+00$ & $2.9 E+01$ \\
$S W$ & $0.0 E+00$ & $0.0 E+00$ & $0.0 E+00$ & $5.9 E+01$ & $4.2 E+01$ & $3.2 E+01$ & $1.8 E+01$ \\
$S S W$ & $0.0 E+00$ & $0.0 E+00$ & $0.0 E+00$ & $0.0 E+00$ & $7.0 E+01$ & $0.0 E+00$ & $2.8 E+01$ \\
$S$ & $0.0 E+00$ & $0.0 E+00$ & $1.3 E+02$ & $0.0 E+00$ & $0.0 E+00$ & $0.0 E+00$ & $0.0 E+00$ \\
$S S E$ & $0.0 E+00$ & $0.0 E+00$ & $0.0 E+00$ & $0.0 E+00$ & $0.0 E+00$ & $0.0 E+00$ & $2.4 E+01$ \\
$S E$ & $0.0 E+00$ & $0.0 E+00$ & $0.0 E+00$ & $1.3 E+02$ & $0.0 E+00$ & $0.0 E+00$ & $3.6 E+01$ \\
$E S E$ & $0.0 E+00$ & $0.0 E+00$ & $0.0 E+00$ & $2.0 E+02$ & $0.0 E+00$ & $1.1 E+02$ & $5.7 E+01$ \\
$E$ & $0.0 E+01$ & $0.0 E+00$ & $0.0 E+00$ & $1.4 E+02$ & $0.0 E+00$ & $0.0 E+00$ & $0.0 E+00$ \\
$E N E$ & $4.4 E$ & $0.0 E$ \\
$N E$ & $0.0 E+00$ & $0.0 E+00$ & $0.0 E+00$ & $0.0 E+00$ & $0.0 E+00$ & $0.0 E+00$ & $0.0 E+00$ \\
$N N E$ & $0.0 E+00$ & $0.0 E+00$ & $0.0 E+00$ & $0.0 E+00$ & $0.0 E+00$ & $0.0 E+00$ & $0.0 E+00$ \\
& & & & & & & \\
\hline
\end{tabular}

Distance (m)

\begin{tabular}{rcccccc} 
Direction & 15000 & 25000 & 35000 & 45000 & $\ddots 55000$ & 70000 \\
\hline & & & & & & \\
$N$ & $0.0 E+00$ & $0.0 E+00$ & $0.0 E+00$ & $0.0 E+00$ & $0.0 E+00$ & $0.0 E+00$ \\
$N N W$ & $0.0 E+00$ & $0.0 E+00$ & $0.0 E+00$ & $0.0 E+00$ & $0.0 E+00$ & $0.0 E+00$ \\
$N W$ & $0.0 E+00$ & $0.0 E+00$ & $0.0 E+00$ & $0.0 E+00$ & $0.0 E+00$ & $0.0 E+00$ \\
$W N W$ & $0.0 E+00$ & $0.0 E+00$ & $0.0 E+00$ & $0.0 E+00$ & $0.0 E+00$ & $0.0 E+00$ \\
$W$ & $6.8 E+00$ & $0.0 E+00$ & $0.0 E+00$ & $0.0 E+00$ & $0.0 E+00$ & $0.0 E+00$ \\
$W S W$ & $1.0 E+01$ & $5.3 E+00$ & $3.4 E+00$ & $2.5 E+00$ & $1.9 E+00$ & $1.4 E+00$ \\
$S W$ & $1.2 E+01$ & $6.0 E+00$ & $3.8 E+00$ & $2.7 E+00$ & $2.1 E+00$ & $1.5 E+00$ \\
$S S W$ & $7.4 E+00$ & $3.8 E+00$ & $2.5 E+00$ & $1.8 E+00$ & $1.4 E+00$ & $1.1 E+00$ \\
$S$ & $1.2 E+01$ & $6.1 E+00$ & $3.9 E+00$ & $2.8 E+00$ & $2.1 E+00$ & $1.6 E+00$ \\
$S S E$ & $9.5 E+00$ & $4.8 E+00$ & $3.1 E+00$ & $2.3 E+00$ & $1.8 E+00$ & $1.3 E+00$ \\
$S E$ & $1.0 E+01$ & $5.0 E+00$ & $3.2 E+00$ & $2.3 E+00$ & $1.8 E+00$ & $1.3 E+00$ \\
$E S E$ & $1.5 E+01$ & $7.2 E+00$ & $4.6 E+00$ & $3.2 E+00$ & $2.4 E+00$ & $1.8 E+00$ \\
$E$ & $2.3 E+01$ & $1.1 E+01$ & $7.0 E+00$ & $4.9 E+00$ & $3.6 E+00$ & $2.6 E+00$ \\
$E N E$ & $1.7 E+01$ & $8.4 E+00$ & $5.3 E+00$ & $3.7 E+00$ & $2.8 E+00$ & $2.0 E+00$ \\
$N E$ & $0.0 E+00$ & $0.0 E+00$ & $0.0 E+00$ & $0.0 E+00$ & $0.0 E+00$ & $0.0 E+00$ \\
$N N E$ & $0.0 E+00$ & $0.0 E+00$ & $0.0 E+00$ & $0.0 E+00$ & $0.0 E+00$ & $0.0 E+00$
\end{tabular}


COLLECTIVE EFFECTIVE DOSE EQUIVALENT (person rem/y) (All Radionuclides and Pathways)

Distance (m)

$\begin{array}{llllllll}\text { nirection } & 310 & 810 & 1500 & 2500 & 3500 & 4500 & 7500\end{array}$

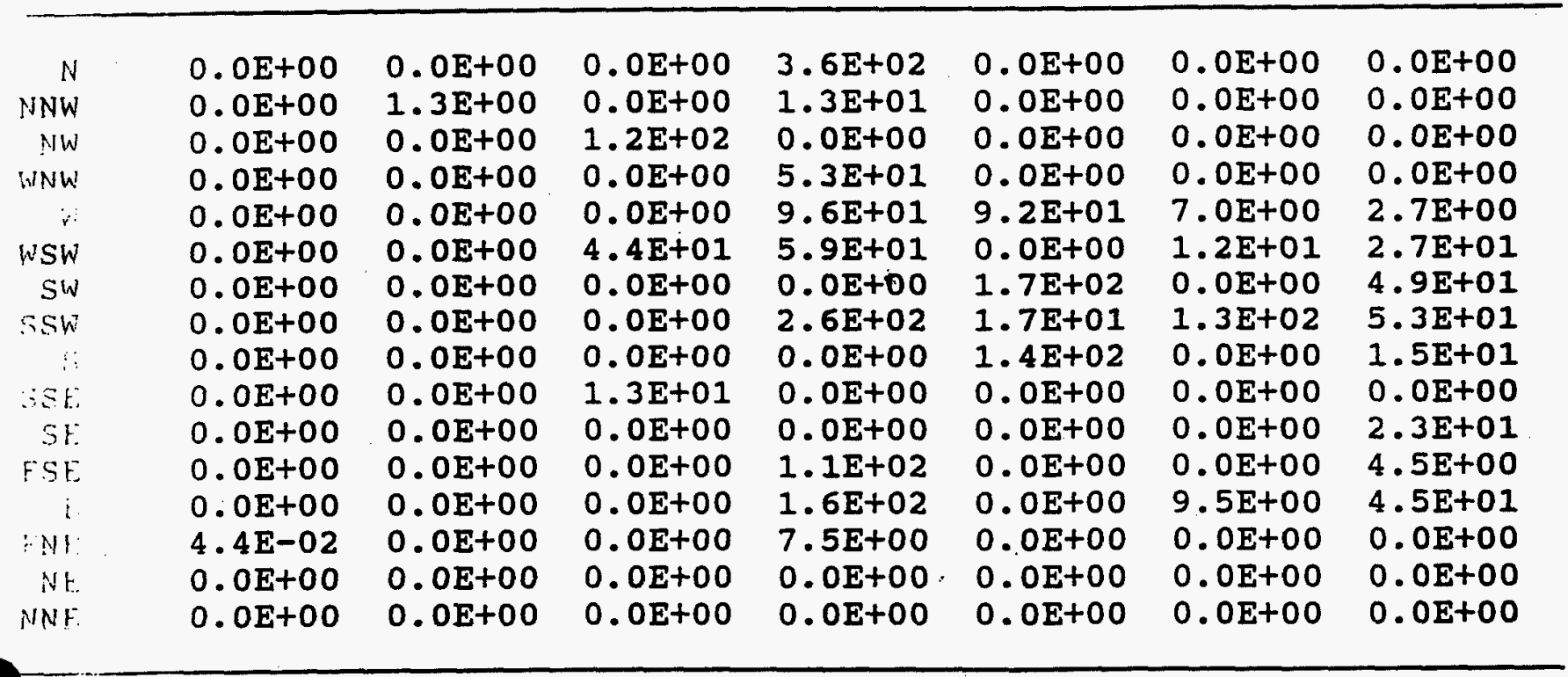

Distance (m)

\begin{tabular}{|c|c|c|c|c|c|c|}
\hline Dirertion & 15000 & 25000 & 35000 & 45000 & 55000 & 70000 \\
\hline ! & $0.0 E+00$ & $0.0 E+00$ & $0.0 E+00$ & $0.0 \mathrm{E}+00$ & $0.0 E+00$ & $0.0 \mathrm{E}+00$ \\
\hline$\because \mathrm{NW}$ & $0.0 E+00$ & $0.0 E+00$ & $0.0 E+00$ & $0.0 E+00$ & $0.0 \mathrm{E}+00$ & $0.0 \mathrm{E}+00$ \\
\hline VW & $0.0 \mathrm{E}+00$ & $0.0 E+00$ & $0.0 E+00$ & $0.0 E+00$ & $0.0 \mathrm{E}+00$ & $0.0 \mathrm{E}+00$ \\
\hline$\because \mathrm{NM}^{\prime}$ & $0.0 \mathrm{E}+00$ & $0.0 \mathrm{E}+00$ & $0.0 \mathrm{E}+00$ & $0.0 \mathrm{E}+00$ & $0.0 \mathrm{E}+00$ & $0.0 \mathrm{E}+00$ \\
\hline w & $2.6 \mathrm{E}+00$ & $0.0 E+00$ & $0.0 E+00$ & $0.0 \mathrm{E}+00$ & $0.0 \mathrm{E}+00$ & $0.0 E+00$ \\
\hline WSW & $8.1 E+00$ & $8.2 E+01$ & $3.0 E+01$ & $1.2 E+02$ & 1. $2 \mathrm{E}+02$ & $4.4 \mathrm{E}+02$ \\
\hline SW & $4.1 E+01$ & 2. $1 E+01$ & $2.9 E+01$ & $1.9 \mathrm{E}+01$ & 4. $9 E+01$ & $2.8 E+02$ \\
\hline SSW & $2.3 E+01$ & 1. $3 E+01$ & $7.7 \mathrm{E}+00$ & 1. $2 \mathrm{E}+01$ & 1. $3 E+01$ & $2.9 E+01$ \\
\hline $\mathrm{S}$ & $9.9 \mathrm{E}+01$ & 1. $4 \mathrm{E}+01$ & $9.3 E+00$ & $1.6 \mathrm{E}+01$ & 1. $7 \mathrm{E}+01$ & $2.2 \mathrm{E}+02$ \\
\hline SSE & $9.3 \mathrm{E}+00$ & $8.7 E+00$ & 1. $1 E+01$ & $8.5 E+00$ & 1. $3 \mathrm{E}+01$ & 1. $1 \mathrm{E}+02$ \\
\hline SE & 1. $4 \mathrm{E}+01$ & 1. $0 E+01$ & 1. $0 E+01$ & $1.2 \mathrm{E}+01$ & $7.8 \mathrm{E}+00$ & 2. $0 E+01$ \\
\hline ESE & $4.6 E+01$ & 6. $0 E+00$ & $1.5 \mathrm{E}+01$ & $1.2 \mathrm{E}+01$ & $6.1 E+01$ & 2. $7 E+01$ \\
\hline $\mathrm{E}$ & $6.1 \mathrm{E}+00$ & $1.9 \mathrm{E}+01$ & $3.8 \mathrm{E}+01$ & $6.5 \mathrm{E}+00$ & 4. $1 E+01$ & $4.6 \mathrm{E}+01$ \\
\hline ENE & $2.5 E+02$ & $5.3 E+01$ & 3. $2 E+01$ & $5.0 \mathrm{E}+01$ & 1. $3 E+02$ & $3.0 \mathrm{E}+02$ \\
\hline NE. & $0.0 E+00$ & $0.0 E+00$ & $0.0 E+00$ & $0.0 E+00$ & $0.0 \mathrm{E}+00$ & $0.0 E+00$ \\
\hline$M N F$ & $0.0 E+00$ & $0.0 E+00$ & $0.0 E+00$ & $0.0 \mathrm{E}+00$ & $0.0 E+00$ & $0.0 \mathrm{E}+00$ \\
\hline
\end{tabular}


INDIVIDUAL LIFETIME RISK (deaths)

(AIl Radionuclides and Pathways)

Distance (m)

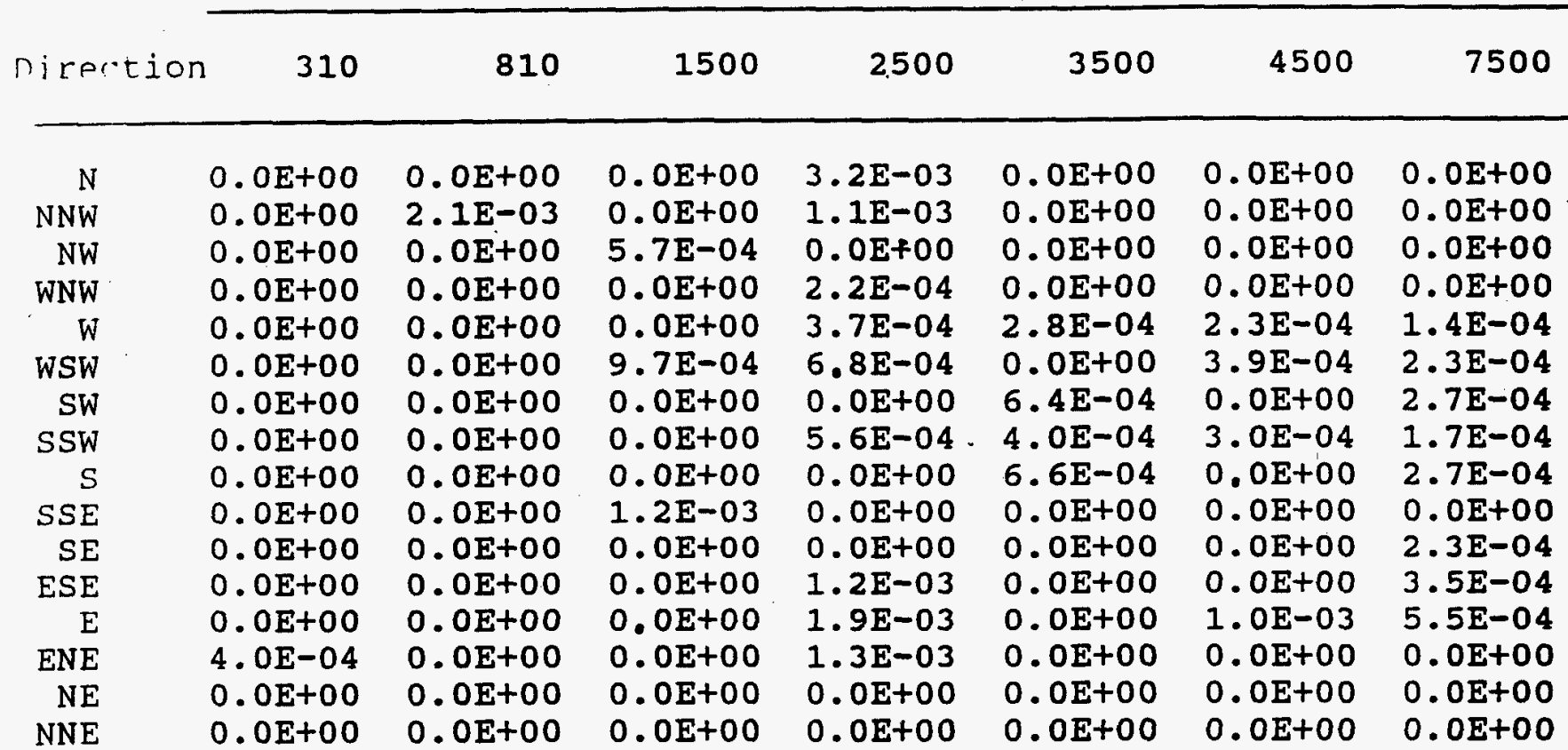

Distance (m)

$\begin{array}{rrrrrrrr}\text { Direction } & 15000 & 25000 & 35000 & 45000 & 55000 & 70000 \\ & & & & & & \\ N & 0.0 E+00 & 0.0 E+00 & 0.0 E+00 & 0.0 E+00 & 0.0 E+00 & 0.0 E+00 \\ \text { NNW } & 0.0 E+00 & 0.0 E+00 & 0.0 E+00 & 0.0 E+00 & 0.0 E+00 & 0.0 E+00 \\ N W & 0.0 E+00 & 0.0 E+00 & 0.0 E+00 & 0.0 E+00 & 0.0 E+00 & 0.0 E+00 \\ \text { WNW } & 0.0 E+00 & 0.0 E+00 & 0.0 E+00 & 0.0 E+00 & 0.0 E+00 & 0.0 E+00 \\ W & 6.3 E-05 & 0.0 E+00 & 0.0 E+00 & 0.0 E+00 & 0.0 E+00 & 0.0 E+00 \\ \text { WSW } & 9.8 E-05 & 5.0 E-05 & 3.2 E-05 & 2.2 E-05 & 1.7 E-05 & 1.2 E-05 \\ \text { SW } & 1.1 E-04 & 5.7 E-05 & 3.6 E-05 & 2.5 E-05 & 1.9 E-05 & 1.3 E-05 \\ \text { SSW } & 7.0 E-05 & 3.5 E-05 & 2.2 E-05 & 1.6 E-05 & 1.2 E-05 & 8.6 E-06 \\ S & 1.1 E-04 & 5.7 E-05 & 3.6 E-05 & 2.6 E-05 & 1.9 E-05 & 1.4 E-05 \\ \text { SSE } & 9.0 E-05 & 4.5 E-05 & 2.9 E-05 & 2.0 E-05 & 1.5 E-05 & 1.1 E-05 \\ \text { SE } & 9.5 E-05 & 4.7 E-05 & 3.0 E-05 & 2.1 E-05 & 1.6 E-05 & 1.1 E-05 \\ \text { ESE } & 1.4 E-04 & 6.9 E-05 & 4.3 E-05 & 3.0 E-05 & 2.2 E-05 & 1.6 E-05 \\ E & 2.2 E-04 & 1.1 E-04 & 6.7 E-05 & 4.6 E-05 & 3.4 E-05 & 2.4 E-05 \\ \text { ENE } & 1.6 E-04 & 8.0 E-05 & 5.0 E-05 & 3.5 E-05 & 2.6 E-05 & 1.8 E-05 \\ N E & 0.0 E+00 & 0.0 E+00 & 0.0 E+00 & 0.0 E+00 & 0.0 E+00 & 0.0 E+00 \\ \text { NNE } & 0.0 E+00 & 0.0 E+00 & 0.0 E+00 & 0.0 E+00 & 0.0 E+00 & 0.0 E+00\end{array}$


COLLECTIVE FATAL CANCER RATE (deaths/Y)

(All Radionuclides and Pathways)

\section{Distance (m)}

Direction $\quad 310$

810

1500

2500

3500

7500

\begin{tabular}{rlllllll}
\hline$N$ & $0.0 E+00$ & $0.0 E+00$ & $0.0 E+00$ & $4.8 E-02$ & $0.0 E+00$ & $0.0 E+00$ & $0.0 E+00$ \\
$N N W$ & $0.0 E+00$ & $1.8 E-04$ & $0.0 E+00$ & $1.7 E-03$ & $0.0 E+00$ & $0.0 E+00$ & $0.0 E+00$ \\
$N W$ & $0.0 E+00$ & $0.0 E+00$ & $1.6 E-02$ & $0.0 E+00$ & $0.0 E+00$ & $0.0 E+00$ & $0.0 E+00$ \\
$W N W$ & $0.0 E+00$ & $0.0 E+00$ & $0.0 E+00$ & $6.9 E-03$ & $0.0 E+00$ & $0.0 E+00$ & $0.0 E+00$ \\
$W$ & $0.0 E+00$ & $0.0 E+00$ & $0.0 E+00$ & $1.3 E-02$ & $1.2 E-02$ & $9.2 E-04$ & $3.5 E-04$ \\
$W S W$ & $0.0 E+00$ & $0.0 E+00$ & $5.8 E-03$ & $7.8 E-03$ & $0.0 E+00$ & $1.6 E-03$ & $3.5 E-03$ \\
$S W$ & $0.0 E+00$ & $0.0 E+00$ & $0.0 E+00$ & $0.0 E+00$ & $2.2 E-02$ & $0.0 E+00$ & $6.5 E-03$ \\
$S S W$ & $0.0 E+00$ & $0.0 E+00$ & $0.0 E+00$ & $3.4 E-02$ & $2.3 E-03$ & $1.8 E-02$ & $7.1 E-03$ \\
$S$ & $0.0 E+00$ & $0.0 E+00$ & $0.0 E+00$ & $0.0 E+00$ & $1.9 E-02$ & $0.0 E+00$ & $2.0 E-03$ \\
$S S E$ & $0.0 E+00$ & $0.0 E+00$ & $1.7 E-03$ & $0.0 E+00$ & $0.0 E+00$ & $0.0 E+00$ & $0.0 E+00$ \\
$S E$ & $0.0 E+00$ & $0.0 E+00$ & $0.0 E+00$ & $0.0 E+00$ & $0.0 E+00$ & $0.0 E+00$ & $3.1 E-03$ \\
$E S E$ & $0.0 E+00$ & $0.0 E+00$ & $0.0 E+00$ & $1.5 E-02$ & $0.0 E+00$ & $0.0 E+00$ & $6.1 E-04$ \\
$E$ & $0.0 E+00$ & $0.0 E+00$ & $0.0 E+00$ & $2.1 E-02$ & $0.0 E+00$ & $1.3 E-03$ & $6.1 E-03$ \\
$E N E$ & $5.7 E-06$ & $0.0 E+00$ & $0.0 E+00$ & $1.0 E-03$ & $0.0 E+00$ & $0.0 E+00$ & $0.0 E+00$ \\
$N E$ & $0.0 E+00$ & $0.0 E+00$ & $0.0 E+00$ & $0.0 E+00$ & $0.0 E+00$ & $0.0 E+00$ & $0.0 E+00$ \\
$N N E$ & $0.0 E+00$ & $0.0 E+00$ & $0.0 E+00$ & $0.0 E+00$ & $0.0 E+00$ & $0.0 E+00$ & $0.0 E+00$ \\
$N$ & & & & & & & \\
\hline
\end{tabular}

Distance (m)

$\begin{array}{lllllll}\text { Direction } & 15000 & 25000 & 35000 & 45000 & 55000 & 70000\end{array}$

\begin{tabular}{rllllll}
\hline$N$ & $0.0 \mathrm{E}+00$ & $0.0 \mathrm{E}+00$ & $0.0 \mathrm{E}+00$ & $0.0 \mathrm{E}+00$ & $0.0 \mathrm{E}+00$ & $0.0 \mathrm{E}+00$ \\
$\mathrm{~N}$ & $0.0 \mathrm{E}+00$ & $0.0 \mathrm{E}+00$ & $0.0 \mathrm{E}+00$ & $0.0 \mathrm{E}+00$ & $0.0 \mathrm{E}+00$ & $0.0 \mathrm{E}+00$ \\
$\mathrm{NW}$ & $0.0 \mathrm{E}+00$ & $0.0 \mathrm{E}+00$ & $0.0 \mathrm{E}+00$ & $0.0 \mathrm{E}+00$ & $0.0 \mathrm{E}+00$ & $0.0 \mathrm{E}+00$ \\
$\mathrm{WNW}$ & $0.0 \mathrm{E}+00$ & $0.0 \mathrm{E}+00$ & $0.0 \mathrm{E}+00$ & $0.0 \mathrm{E}+00$ & $0.0 \mathrm{E}+00$ & $0.0 \mathrm{E}+00$ \\
$\mathrm{~W}$ & $3.4 \mathrm{E}-04$ & $0.0 \mathrm{E}+00$ & $0.0 \mathrm{E}+00$ & $0.0 \mathrm{E}+00$ & $0.0 \mathrm{E}+00$ & $0.0 \mathrm{E}+00$ \\
$\mathrm{WSW}$ & $1.1 \mathrm{E}-03$ & $1.1 \mathrm{E}-02$ & $3.9 \mathrm{E}-03$ & $1.5 \mathrm{E}-02$ & $1.5 \mathrm{E}-02$ & $5.3 \mathrm{E}-02$ \\
$\mathrm{SW}$ & $5.5 \mathrm{E}-03$ & $2.8 \mathrm{E}-03$ & $3.8 \mathrm{E}-03$ & $2.5 \mathrm{E}-03$ & $6.2 \mathrm{E}-03$ & $3.5 \mathrm{E}-02$ \\
$\mathrm{SSW}$ & $3.0 \mathrm{E}-03$ & $1.7 \mathrm{E}-03$ & $9.9 \mathrm{E}-04$ & $1.5 \mathrm{E}-03$ & $1.5 \mathrm{E}-03$ & $3.3 \mathrm{E}-03$ \\
$\mathrm{~S}$ & $1.3 \mathrm{E}-02$ & $1.8 \mathrm{E}-03$ & $1.2 \mathrm{E}-03$ & $2.1 \mathrm{E}-03$ & $2.1 \mathrm{E}-03$ & $2.7 \mathrm{E}-02$ \\
$\mathrm{SSE}$ & $1.3 \mathrm{E}-03$ & $1.1 \mathrm{E}-03$ & $1.5 \mathrm{E}-03$ & $1.1 \mathrm{E}-03$ & $1.6 \mathrm{E}-03$ & $1.3 \mathrm{E}-02$ \\
$\mathrm{SE}$ & $1.9 \mathrm{E}-03$ & $1.4 \mathrm{E}-03$ & $1.3 \mathrm{E}-03$ & $1.5 \mathrm{E}-03$ & $9.8 \mathrm{E}-04$ & $2.4 \mathrm{E}-03$ \\
$\mathrm{ESE}$ & $6.3 \mathrm{E}-03$ & $8.1 \mathrm{E}-04$ & $2.0 \mathrm{E}-03$ & $1.6 \mathrm{E}-03$ & $7.9 \mathrm{E}-03$ & $3.4 \mathrm{E}-03$ \\
$\mathrm{E}$ & $8.3 \mathrm{E}-04$ & $2.6 \mathrm{E}-03$ & $5.1 \mathrm{E}-03$ & $8.7 \mathrm{E}-04$ & $5.4 \mathrm{E}-03$ & $6.0 \mathrm{E}-03$ \\
$\mathrm{ENE}$ & $3.4 \mathrm{E}-02$ & $7.2 \mathrm{E}-03$ & $4.2 \mathrm{E}-03$ & $6.5 \mathrm{E}-03$ & $1.7 \mathrm{E}-02$ & $3.8 \mathrm{E}-02$ \\
$\mathrm{NE}$ & $0.0 \mathrm{E}+00$ & $0.0 \mathrm{E}+00$ & $0.0 \mathrm{E}+00$ & $0.0 \mathrm{E}+00$ & $0.0 \mathrm{E}+00$ & $0.0 \mathrm{E}+00$ \\
$\mathrm{NNE}$ & $0.0 \mathrm{E}+00$ & $0.0 \mathrm{E}+00$ & $0.0 \mathrm{E}+00$ & $0.0 \mathrm{E}+00$ & $0.0 \mathrm{E}+00$ & $0.0 \mathrm{E}+00$
\end{tabular}


M98004259

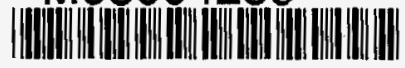

Report Number (14) FEMP $=2552$

Publ. Date (11) 199712

Sponsor Code (18) DO \& IDP, XF

UC Category (19) UC-702, DOE/ER

DOE 